\title{
Validation of a new technique of production data analysis for single and multi-layer formations under controlled environment
}

\author{
Domingo A. Mata \\ West Virginia University
}

Follow this and additional works at: https://researchrepository.wvu.edu/etd

\footnotetext{
Recommended Citation

Mata, Domingo A., "Validation of a new technique of production data analysis for single and multi-layer formations under controlled environment" (2007). Graduate Theses, Dissertations, and Problem Reports. 1834.

https://researchrepository.wvu.edu/etd/1834

This Thesis is protected by copyright and/or related rights. It has been brought to you by the The Research Repository @ WVU with permission from the rights-holder(s). You are free to use this Thesis in any way that is permitted by the copyright and related rights legislation that applies to your use. For other uses you must obtain permission from the rights-holder(s) directly, unless additional rights are indicated by a Creative Commons license in the record and/ or on the work itself. This Thesis has been accepted for inclusion in WVU Graduate Theses, Dissertations, and Problem Reports collection by an authorized administrator of The Research Repository @ WVU. For more information, please contact researchrepository@mail.wvu.edu.
} 


\title{
VALIDATION OF A NEW TECHNIQUE OF PRODUCTION DATA ANALYSIS FOR SINGLE AND MULTI-LAYER FORMATIONS UNDER CONTROLLED ENVIRONMENT
}

Domingo A. Mata

Thesis submitted to the College of Engineering and Mineral Resources at West Virginia University in partial fulfillment of the requirements for the degree of

\author{
Master of Science \\ in Petroleum and Natural Gas Engineering
}
Shahab D. Mohaghegh, Ph.D., Chair. Razi Gaskari, Ph.D. Khashayar Aminian, Ph. D.
Department of Petroleum and Natural Gas Engineering Morgantown, West Virginia 2007

Keywords: reservoir simulation, production data analysis, intelligent systems. 


\begin{abstract}
VALIDATION OF A NEW TECHNIQUE OF PRODUCTION DATA ANALYSIS FOR SINGLE AND MULTI-LAYER FORMATIONS UNDER CONTROLLED ENVIRONMENT
\end{abstract}

Domingo A. Mata

\begin{abstract}
Intelligent Production Data Analysis - IPDA, is a new methodology for Reservoir Characterization based only on monthly production rate data. This technique combines conventional methods of production data analysis (decline curve analysis, type curve matching and history matching) with intelligent systems. The study targets the validation of this methodology under a controlled environment, attempting three main objectives: Identifying Sweet Spots, Forecasting Reserves and recognizing under-performer wells.
\end{abstract}

The study investigates the behavior of five different reservoirs, modeled using a commercial simulator. The structure, parameters and heterogeneity of each configuration was inspired by existing formations. Records of production rate data were generated from the simulated fields (both single and multi-layer formations) and used as input to perform an "Intelligent Production Data Analysis".

The findings highlight strength of this technique in tracking the fluid movement in the reservoir as a function of time. Furthermore, this study identifies some limitations and circumstances under which the analysis may not result in correct recommendations. 


\section{ACKNOWLEDGEMENTS}

First, I must thank God for guiding my steps and providing me with health and strength along the way.

To my parents, Dr. Domingo J. Mata and Dr. Guadalupe Madrid for tlıı continuous cooperation and wise advice. Without their support this would not have been possible.

To my sister and brother, Maria F. Mata and Francisco J. Mata. They are always my inspiration.

To my research advisors, Dr. Razi Gaskari and Dr. Shahab Mohaghegh. They have guided me through this project with commitment and patience. They represented a model to follow and a continuous source for apprenticeship. I will never forget what they have done for me. They have won my eternal respect.

To my research team members, Jalal Jalali, Miguel Tovar, Daniel Gonzalez, Delal Gunaydin, Cesar Silva and Kazim Malik. Under any circumstances they have been there supporting me. I wish you all the best.

To Professor Sam Ameri, Dr. Khashayar Aminian, Dr. Ilkin Bilgesu and Beverly Matheny for their cooperation throughout this program. 


\section{Table of Contents}

Abstract

Acknowledgements iii

Table of Contents iv

List of Tables $\quad$ vi

List of Figures vii

Chapter I - Introduction $\quad 1$

1.1 Problem Statement 3

Chapter II - Theoretical Framework $\quad 4$

2.1 Literature Review 4

2.2 Decline Curve Analysis 11

2.3 Type Curve Matching 13

2.4 History Matching 16

2.5 Intelligent Production Data Analysis 18

2.5.1 Data Acquisition 19

2.5.2 Decline Curve Analysis 20

2.5.3 Type Curve Matching 21

2.5.4 History Matching 23

2.5.5 Field-wide Pattern Recognition 25

2.5.6 Under-Performer wells 27

2.5.7 Evaluation of What-if Scenarios 29

Chapter III - Methodology 33

3.1 Modeling a reservoir in a controlled environment 35

3.1.1 Single Layer Model 36

3.1.2 Two-Layer Model 38

3.2 Schedule of Production 41

3.3 Performing the IPDA analysis 45 
3.3.1 Decline Curve Analysis 46

3.3.2 Type Curve Matching 47

3.3.3 History Matching 47

3.3.4 Field-wide Pattern Recognition $\quad 49$

3.4 Evaluation of Results 53

3.4.1 First set of results - Reservoir properties 53

3.4.2 Second set of results - Well properties 53

3.4.3 Third set of results - Well performance 54

Chapter IV - Results and Recommendations $\quad \mathbf{5 8}$

4.1 First set of result - Reservoir properties 60

4.1.1 First set of results for Single Layer Model 60

4..1.2 First set of results for Two-Layer Model 71

4.2 Second set of results - Well properties 83

4.3 Third set of results - Well performance 85

4.3.1 Under-performer wells 85

4.3.2 Evaluation of "what-if scenarios" $\quad 87$

Chapter V - Conclusions and Recommendations 93

$\begin{array}{ll}\text { References } & 95\end{array}$

$\begin{array}{ll}\text { Appendix A } & 96\end{array}$

Appendix B 100

Appendix C 103 


\section{List of Tables}

Table 1 -Data organized to be imported in IPDA. 20

Table 2 - Characteristics of the Single Layer Model. 37

Table 3 - Characteristics of the Two-Layer Model. 39

Table 4 - Reservoir Versions $\quad 40$

Table 5 - Drilling schedule for 100 wells $\quad 42$

Table 6 - Economic analysis on well \# $8 \quad 90$

Table 7 - Economic analysis on well \# 5 


\section{List of Figures}

Fig. 1 - Principle of IPDA technique. $\quad 8$

Fig. 2 - Cox Approach for Type Curve Matching 14

Fig. 3 - Principle of History Matching 16

Fig. 4 - Initial stage of Decline Curve Analysis 21

Fig. 5 - Type Curve Matching $\quad 22$

Fig. 6 - Flow-Chart iterative process $\quad 24$

Fig. 7 - Relative Reservoir Quality Index 26

Fig. 8-3d Surface Plot $\quad 27$

Fig. 9 - Under-Performer Wells $\quad 29$

Fig. 10 - Location of a future well $\quad 30$

Fig. 11 - Predicted behavior of an infill drilled well. 32

Fig. 12 - Flow Chart of the validation of IPDA technique 33

Fig. 13 - Grid Block Matrix $\quad 35$

Fig. 14 - SLM 224. Well Distribution 37

Fig. 15 - SLM 100. Well Distribution 38

Fig. 16 - Well distribution of Two-Layer Models. $\quad 40$

Fig. 17 - Production Rate vs. Time in neighbor wells 43

Fig. 18 -Final match of a Decline Curve Analysis. 46

Fig 19 - Initial Conditions for History Matching 48

Fig. 20 - Successful History Match $\quad 49$

Fig. 21 - Revision of an appropriate partitioning 51

Fig. 22 - Well distribution SLM 100

Fig. 23 - Location of the new wells 56

Fig. 24 - SLM. Actual Initial Gas Distribution 60

Fig. 25 - RRQI SLM224. Initial Gas Distribution 61

Fig. 26 - RRQI SLM100 Initial Gas Distribution 62

Fig. 27 - SLM. Actual Remaining Gas Distribution 63

Fig. 28 - RRQI SLM224. Remaining gas distribution after 30 years 64

Fig. 29 - RRQI SLM100. Remaining gas distribution after 30 years 65 
Fig. 30 - SLM224. Evaluation of 45 years EUR 66

Fig. 31 - SLM100. Evaluation of 45 years EUR 67

Fig. 32 - SLM. Permeability 68

Fig. 33a-RRQI SLM224. Permeability Distribution 69

Fig. 33b -SLM 224. Permeability Distribution. 70

Fig. 34 - SLM 100 Permeability trend. 71

Fig. 35 - TLM-1. Actual Initial gas distribution $\quad 72$

Fig. 36 - 3D Surface TLM-1. Initial gas distribution 73

Fig. 37 - TLM-2 \& TLM-3. Actual Initial gas distribution 73

Fig. 38 - RRQI TLM-2. Initial gas distribution $\quad 74$

Fig. 39 - TLM-1. Actual gas distribution at 26 years 75

Fig. 40 - RRQI TLM-1. Gas distribution after 26 years 75

Fig. 41 - TLM-2. Gas distribution after 26 years $\quad 76$

Fig. 42 - TLM-3. Gas distribution after 26 years $\quad 77$

Fig. 43 - RRQI TLM-3. Gas distribution after 26 years 78

Fig. 44 - TLM-1. Evaluation of 45 years EUR $\quad 79$

Fig. 45 - TLM-1 \&TLM-3. Actual Permeability. 80

Fig. 46 - TLM-1 \& TLM-3. Permeability (IPDA) 81

Fig. 47 - TLM-2. Permeability (IPDA) 82

Fig. 48 - SLM 100. Drainage Area (IPDA) 83

Fig. 49 - Drainage area distribution (with skin) 84

Fig. 50 - Under-performer wells. $\quad 85$

Fig. 51 - Impact of reservoir boundaries on well performance. 86

Fig. 52 - SLM100. Well Distribution. 87

Fig. 53 - Locations selected for infill drilling 88

Fig. 54 - Gas flow rate vs. time well \# 8

Fig. 55 - Gas flow rate vs. time well \# 5 


\section{Chapter I-Introduction}

A recent rise in the global demand for energy has significantly increased oil and gas prices. In the last few years E\&P companies have reported record profits. A new reality rules the energy market, and large amounts of money have been invested in order to increase the production capacity. Now, mature fields, which were not profitable in the late 90s, have become very attractive for major oil and gas producers. An effective revitalization of this type of reservoirs has come to play a big role in the industry.

Recovery techniques have been tremendously improved over the last decade. However, lack of data is a problem with mature fields. Production Rate data is about the only data that can be easily accessed in most of the brown fields. But, what can be done with this data?

Production Analysis techniques have been on the petroleum engineers' agenda for many years. Several tools and strategies have been developed in this field for estimating remaining reserves and recommending infill drilling locations. However, these tools require large amounts of data records and intrinsic reservoir properties to arrive at any conclusion.

The methods most frequently used for analyzing production data are: decline curve analysis, type curve matching, and history matching. However, each of these methods carries a high amount of subjectivity when applied individually in the context of production data.

Recently, a new technique for production analysis was introduced. The procedure is called Intelligent Production Data Analysis (IPDA). It combines the well-known methods for production data analysis (Decline Curve Analysis, Type Curve Matching and History Matching) with intelligent systems (Neural Networks, Genetic Algorithms and Fuzzy Logic). The 
results provide a unified set of reservoir characteristics based only on records of monthly production rate data.

The aforementioned technique has been successfully applied in several fields throughout the United States. Positive results have increased the interest in evaluating the applicability of IPDA over a wider range of reservoirs. Therefore, it is necessary to verify its accuracy over different types of formations in order to identify the strengths and weaknesses associated with this methodology.

This study will prove that the IPDA technique is capable of mapping fluid flow in the reservoir at any given time. Furthermore, potential locations for infill drilling will be recognized. Finally, the technique will be employed in order to point out those wells that are not performing at full capacity and might become candidates for stimulation procedures. 


\subsection{Statement of the Problem}

The purpose of this study is validating the applicability of IPDA technique in reservoirs of heterogeneous characteristics, including both single and multilayered formations. Furthermore, the accuracy of results will be assessed as well as the potential of this methodology for recognizing new opportunities in a field. Finally, the limitations and circumstances under which IPDA technique might result in incorrect recommendations will also be identified. 


\section{Chapter II - Theoretical Framework}

\subsection{Literature Review}

Production from mature fields, also known as brown fields, started at a time when reservoir characterization was not a priority. An upward trend in the prices of oil and gas has increased the feasibility of revitalizing this type of reservoir. In the majority of the mature fields, the information available is scarce, and the most common type of data that may be found is production rate data. Production rate data is some of the only existing reliable information, because it must be reported to government agencies periodically, and because records of production are necessary to calculate revenues. The question is: what can be done with production rate data?

The analysis of production data has been used in petroleum engineering for decades. So far, these techniques have proven to be quite valuable for individual well assessments. However, when it comes to the characterization of an entire field, results tend to be quite subjective. There are several techniques for production analysis. Three of most frequently-used are: Decline Curve Analysis (DCA), Type Curves Matching (TCM) and History Matching (HM). These methods have improved in quality and applicability throughout the years, because they represent a practical alternative for monitoring performance in a producing field.

Decline Curve Analysis (DCA) is likely the oldest method used in petroleum engineering for analyzing production data. It consists of fitting previous records of production data with a mathematical model. This methodology was introduced by Arps $^{1}$ in the 1940s. DCA, an empirical method which does not require any information regarding the reservoir or well parameters, has been widely used for its simplicity. Today, it remains a frequent practice in petroleum engineering for forecasting future behavior of producing wells. 
Fetkovich ${ }^{2}$ introduced decline curve analysis by type curves in the 1980 s by relating Arps decline parameters to reservoir engineering indicators. This study projected the application of flow-rate type curves in petroleum engineering and several authors evaluated their applicability over different type of reservoirs.

Anderson ${ }^{3}$ provided a set of diagnostic methods in order to verify the applicability of production analysis to observed data. He proved that a successful production analysis depends on the quality and quantity of the data records. $\mathrm{He}$ recognized the applicability of production analysis and its potential in reservoir engineering. He ended his study recommending surveillance of the data acquisition and periodic reviews of the records collected.

At this point, production analysis was recognized as a potential alternative to costly pressure tests. However, the subjectivity issue remained intact. A second major shortcoming was the fact that there was no way to connect the information from individual wells to the reservoir's intrinsic parameters. Many studies attempted to solve these two problems but none of them provided a convincing answer.

An interesting study presented by $\mathrm{Poe}^{4}$ in the late 1990's highlighted the fact that when a production analysis is performed, most of the results will vary according to the technique used, because each methodology has different assumptions. Poe tried to address this issue by developing a comprehensive method for performing different kinds of production analyses over the same records of production data. In addition, he recommended the application of statistical analysis and remediation techniques to facilitate the reduction of outliers in noisy data records. The result proved a notable enhancement in the quality of the estimations of reservoir and well properties. This was a starting point for reducing the subjectivity associated with production analysis. 
A relevant study for field application of production analysis was presented by Agarwal ${ }^{5}$ in 1999. He combined Decline-Curve and Type-Curve Analysis to come up with a tool for better prediction of oil or gas in place, permeability, skin, fracture half-length, conductivity, etc. One of the most interesting parts of Agarwal study was the fact that he verified the accuracy of his method not only with field cases but also with numerical simulation. This was the first effort to corroborate the veracity of the predictions accomplished through production analysis.

It was clear that the combination of different techniques of production analysis could play an important role in detecting inconsistencies and pitfalls associated with these methods. The fact that diverse techniques (each one with different assumptions) produced similar results reduced significantly the chances of erroneous analysis, because in case there was a mistake, it would be detected. However, the second shortcoming related to production analysis (connecting the information from individual wells together) was still not resolved.

What needs to be solved here is a sort of Rubik's cube (a mechanical puzzle invented by a Hungarian professor in 1974) made out of small pictures of reservoir parameters. The strategy should be the same; come up with a standard procedure to deduce the sequence of movements that will solve the puzzle. Poe ${ }^{6}$ tried to achieve this in his next study.

This time, Poe's investigation was specifically applied to fractured wells in low permeability reservoirs, targeting the estimation of the effective permeability in the entire field. He was able to perform an advanced diagnostic of around 200 fractured wells based on production rate data. Additional reservoir properties were collected in a well-to-well basis, parameters such as thickness, porosity, saturation, flowing tubing pressure, initial reservoir pressure and temperature were stored in a data base. Poe stated that the process of data acquisition took 15 man-months. The application of production analysis techniques took 
additional 13 man-months. That illustrates the effort that is required in order to apply the techniques of production analysis in a field-wide approach. $\mathrm{He}$ successfully calculated individual permeability values and he tried to come up with a method to estimate the effective permeability of the reservoir. This was a huge step in the application of Production Analysis. It projected the potential of this method as a valid alternative for reservoir characterization. Nevertheless, the complexity of this methodology made it unfeasible for practical purposes.

The problem at this point was partially solved. It had been proven that the subjectivity of Production Analysis can be reduced by utilizing different methods with the same records of data. It was demonstrated as well, that field-wide characterization of intrinsic properties in a reservoir was possible. However, the limitation was that these procedures involved a tremendous amount of effort. The next step was coming up with a more comprehensive technique to inter-connect individual well analyses. There has got to be an easier way of doing this.

The necessity of finding solutions to non-linear problems pushed the oil and gas industry to explore new technologies that had been proven to work efficiently in other scientific fields. The revolution of intelligent systems represented a valuable opportunity. New computer programs are capable of gathering information from the environment, memorizing the results of their actions and learning from the experience of these results in order to achieve objectives more efficiently. But, are these intelligent systems applicable to petroleum engineering problems?

Mohaghegh ${ }^{7}$ has been focused on answering this query since $1991 . \mathrm{He}$ has proven that intelligent systems can play an important role in solving petroleum engineering problems. After several years of research a possible connection between Production Analysis techniques and intelligent systems was encountered. It solved the limitation associated with the integration of individual results in a unified model. 
The procedure is called Intelligent Production Data Analysis - IPDA. It combines well-known methods for production data analysis (Decline Curve Analysis, Type Curve Matching and History Matching) with intelligent systems (Neural Networks, Genetic Algorithms and Fuzzy Logic). It was introduced by Mohaghegh in 2005, and provides a field-wide reservoir characterization based only on records of monthly production rate data. The result is a set of reservoir characteristics that may be used for evaluating future scenarios in a producing field as well as mapping fluid flow at any given time. (See Fig 1)

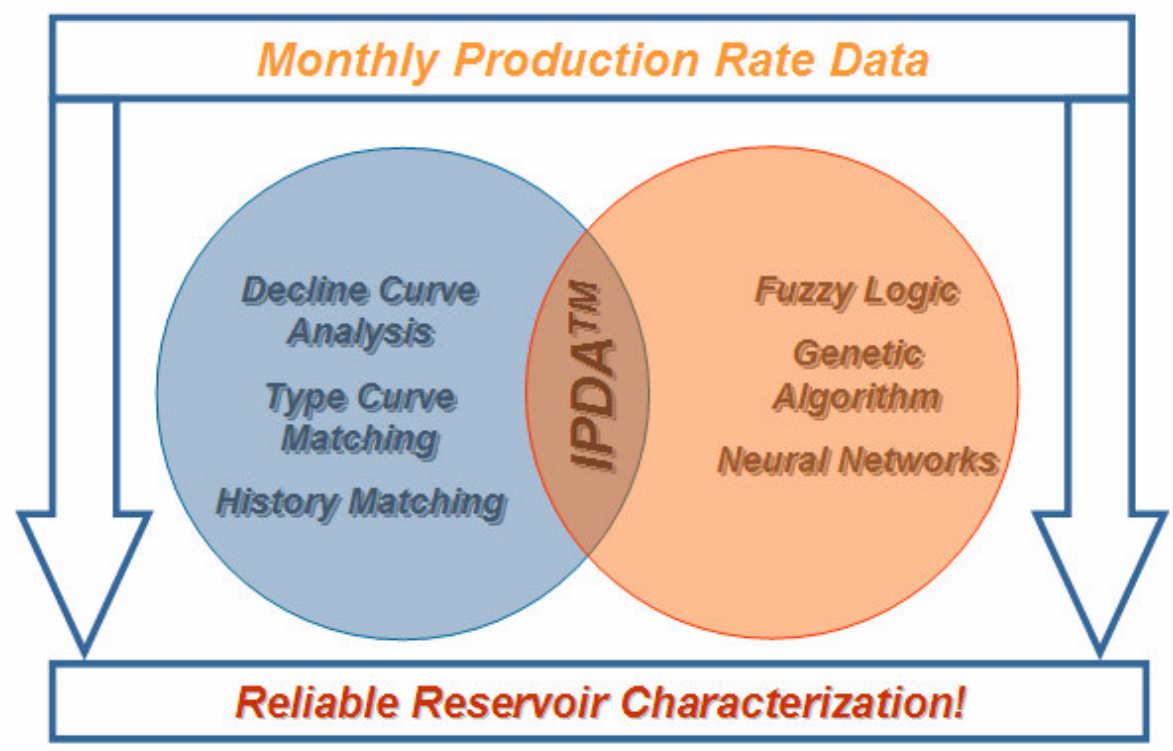

Fig. 1 -Principle of IPDA technique.

This technique significantly reduces the subjectivity associated with conventional techniques by maintaining a close inter-connection every step of the process. Every step is carried out within parameters calculated in previous stages, while always preserving the essence of each technique performed.

The connection of the individual parameters into a unified set of reservoir properties is accomplished through the employment of an intelligent procedure 
called Field-wide Pattern recognition. This step collects the individual well information and deduces a pattern in the data.

The methodology described has been applied in several fields throughout the United States (Rockies and Mid-Continent) obtaining successful results. One of these cases was the Golden Trend fields in Oklahoma, where the only data used to perform the analysis was records of production rate data. Positive results increased interest in evaluating its applicability over a wide range of mature fields. Furthermore, the fact that this information can be found in public records, projects the IPDA as a valuable tool for independent asset evaluation, prior to lease acquisitions.

Another case of successful application of IPDA was presented by Jalali ${ }^{8}$ in 2006. The technique was used for characterizing Carthage Field in the Cotton Valley Formation in Texas. Production records from 349 wells were employed during this analysis. The result was a unified set of reservoir indicators such as, Estimated Ultimate Recovery (EUR), remaining reserves at different times, permeability distribution, drainage area, etc. based only on public records of monthly production rate data, which led to the discovery of a new dimension for production analysis.

Gaskari ${ }^{9}$ applied an Intelligent Production Data Analysis using records from the Wattenberg field producing from Codell and Niobrara formations in the D.J. Basin of Rockies. Gaskari validated the Fuzzy Pattern Recognition as a feasible method to map reservoir characteristics on the entire field. He was able to characterize the reservoir and recognize under-performer wells. Furthermore, using IPDA technique he assessed potential locations for infill drilling.

These findings led to several questions: How reliable is this technique? What are its limitations? What information is required to perform the analysis? What other available information might prove useful? Does this technique work in 
reservoirs with multiple pay zones? In conclusion, it is obligatory to conduct a study that evaluates the reach and limitations of the IPDA technique, as well as its applicability over a wide range of mature fields.

This study attempts to validate the IPDA technique by evaluating the accuracy of its predictions under different reservoir configurations. Single and Multi-layered formations with heterogeneous distribution of reservoir parameters (porosity, permeability, thickness) will be evaluated. To do so, it is necessary to develop different models configured in a controlled environment (to be hosted in a reservoir simulator). Each model will be inspired by existing formations.

Production data will be generated in the simulator and these records will be used as input for the IPDA analysis. A set of reservoir parameters is going to be employed for corroborating the accuracy of the IPDA technique. Initial gas distribution, remaining reserves at different times, and drainage area are examples of variables that will be calculated and compared in order to test the accuracy of the IPDA technique. Finally, it is important to identify those circumstances under which the analysis might result in incorrect recommendations.

In conclusion, the IPDA represents an opportunity for independent producers operating mature fields. In cases where pressure data is non-existent and pressure tests are not feasible (or simply unaffordable) the IPDA provides an alternative for characterizing the reservoir. 


\subsection{Decline Curve Analysis}

Estimating reserves and forecasting reservoir behavior has been a challenge for industry professionals for decades. Different procedures have been developed for this purpose.

One frequently used method is the Decline Curve Analysis. This technique consists of fitting a curve with a mathematical function in order to match observed records of Production Data. Most of the approaches for Decline Curve Analysis are based on the empirical equation developed by Arps in the 1940's. The Arps equation for decline curve analysis establishes a relationship between production rate and time for wells during pseudo-steady state period.

The equation states that:

$$
q_{t}=\frac{q_{i}}{\left(1+b D_{i} t\right)^{1 / b}}
$$

where

$$
\begin{aligned}
& q_{t}=\text { the oil production rate at time } t \text { and } \\
& q_{i}=\text { the initial oil production rate. } \\
& D_{i}=\text { the initial decline rate. } \\
& b=\text { hyperbolic exponent }
\end{aligned}
$$

If $b=0$, the decline is exponential.

If $b=1$, the decline is harmonic.

If $0<b<1$, the decline is hyperbolic.

It is difficult to predict which decline (exponential, hyperbolic or harmonic) the reservoir will follow, and each approach has disadvantages. For example, the 
exponential decline curve tends to underestimate reserves and production rates. On the other hand, the harmonic decline curve has a tendency to overestimate the reservoir performance. In some cases, production decline data do not follow any model but cross over the entire set of curves.

Fetkovich introduced decline curve analysis by type curves in the 1980s by relating Arps decline parameters (Qi,Di and b) to reservoir engineering indicators. He combined the transient rate and the pseudo steady-state decline curves in a single graph. He also related the empirical equations of Arps to single-phase flow solutions and attempted to provide a theoretical basis for the Arps equations.

Many derivations were based on the assumption of single-phase flow in closed boundary systems. Less attention has been paid to the production decline analysis in naturally-fractured reservoirs created by water flooding.

The Arps decline equation is still being used because of its simplicity, since it is an empirical method and does not require any reservoir or well parameters. Several studies on analysis of production decline are still based on this empirical method. Many published papers have tried to interpret the Arps decline equation theoretically. 


\subsection{Type Curve Matching}

Type curve matching is another form of verifying the result obtained from Decline Curve Analysis. This methodology was introduced by Fetkovich and it relates the Arps decline parameters to reservoir engineering indicators.

A type curve is a log-log plot of a family of production decline curves. The plot illustrates the dimensionless flow rate $\left(q_{D}\right)$ on ordinates versus dimensionless time $\left(t_{D}\right)$ on abscissa.

The process of type curve matching consists of fitting observed production data on a series of generated curves. Once an acceptable fit has been found, a match point can be selected so the dimensionless groups may be evaluated. Finally, the matched type curve and the dimensionless groups are utilized to estimate the future production rates. Fetkovich greatly simplified the selection of the appropriate hyperbolic decline exponent $(b)$, which made decline curve analysis easier and faster.

The equation for dimensionless flow rate is:

$$
q_{D}=\frac{141.3 q(t) \mu B}{k h\left(p_{i}-p_{w f}\right)}
$$

The equation for dimensionless time is:

$$
t_{D}=\frac{0.00634 k t}{\phi \mu c_{t} r_{w}^{2}}
$$

Where...

$\begin{array}{lll}q_{D}=\text { dimensionless flow rate } & \mathrm{B}=\text { formation volume factor } & \mathrm{Pi}=\text { average initial pressure } \\ q(t)=\text { flow rate at time } t . & k=\text { permeability } & \mathrm{P}_{\mathrm{wf}}=\text { flowing bottom-hole } \\ \text { pressure }\end{array}$


$\mathrm{C}_{\mathrm{t}}=$ total compressibility

$$
\begin{aligned}
& \varnothing=\text { porosity } \\
& h=\text { thickness }
\end{aligned}
$$$$
r_{w}=\text { wellbore radius }
$$

The limitations of Fetkovich's work on type curves were: the restriction to radial symmetric flow of low-compressibility fluids, and discarding tight reservoirs with low permeability. However, stimulation treatments grew larger, and gradually lower permeability reservoirs started to be developed. Therefore, a further specific approach was necessary for this type of formation.

In 1996 Cox $^{10}$ introduced an advanced type curve analysis specifically applied to low permeability gas reservoirs. This study considers several parameters that affect type curve performance. For example, the effect of reservoir shape, skin, compressibility and viscosity were studied more in detail.

The Cox approach uses flow rate type curves (at constant pressure) developed specifically for low permeability gas reservoirs with hydraulically fractured wells. The methodology is based on the hyperbolic exponent (b). For a particular $b$ value Cox created a set of type curves in which each curve had a different value for $\mathrm{Xe} / \mathrm{Xf}$. The first curve represented an $\mathrm{Xe} / \mathrm{Xf}$ factor of one, and it progressively increased until reaching until an infinite value for $\mathrm{Xe} / \mathrm{Xf}$ in the top curve (See Fig. 2).
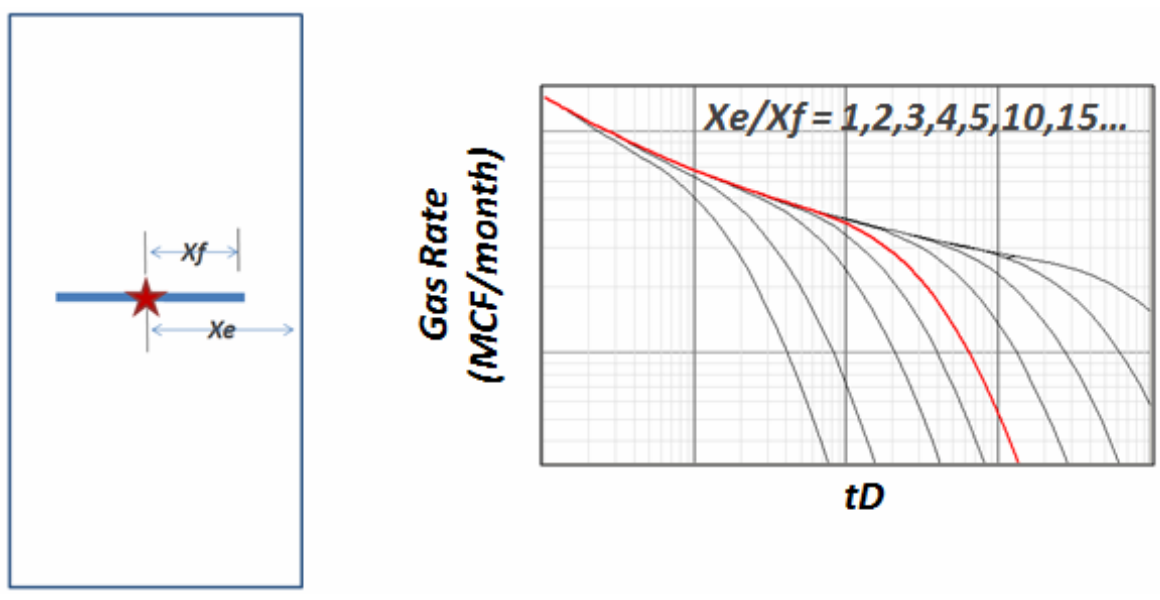

$t D$

Fig. 2 - Cox approach for type curve matching 
Finally, Cox had developed a methodology that incorporated reservoir geometry and isotropicity, skin effect, and varying compressibility with Fetkovich equations. This provided engineers a new and easy tool for assessing well performance and infill drilling in tight gas reservoirs. 


\subsection{History Matching}

The act of adjusting various parameters in a modeled reservoir until it reproduces past behavior of observed data is known as History Matching. In this research, this process was conducted for each well using a Single-Well Radial Model.

In Fig. 3, the principle of history matching is explained. In the Single Radial Well Model (left), various parameters, such as Permeability, Porosity, Initial Pressure, Drainage Area, Temperature, Gas Saturation, etc. can be adjusted until the observed data is successfully matched. The right picture in Fig. 3 illustrates an initial guess in a history matching procedure; the green line represents the real data and the red line is the simulated behavior.
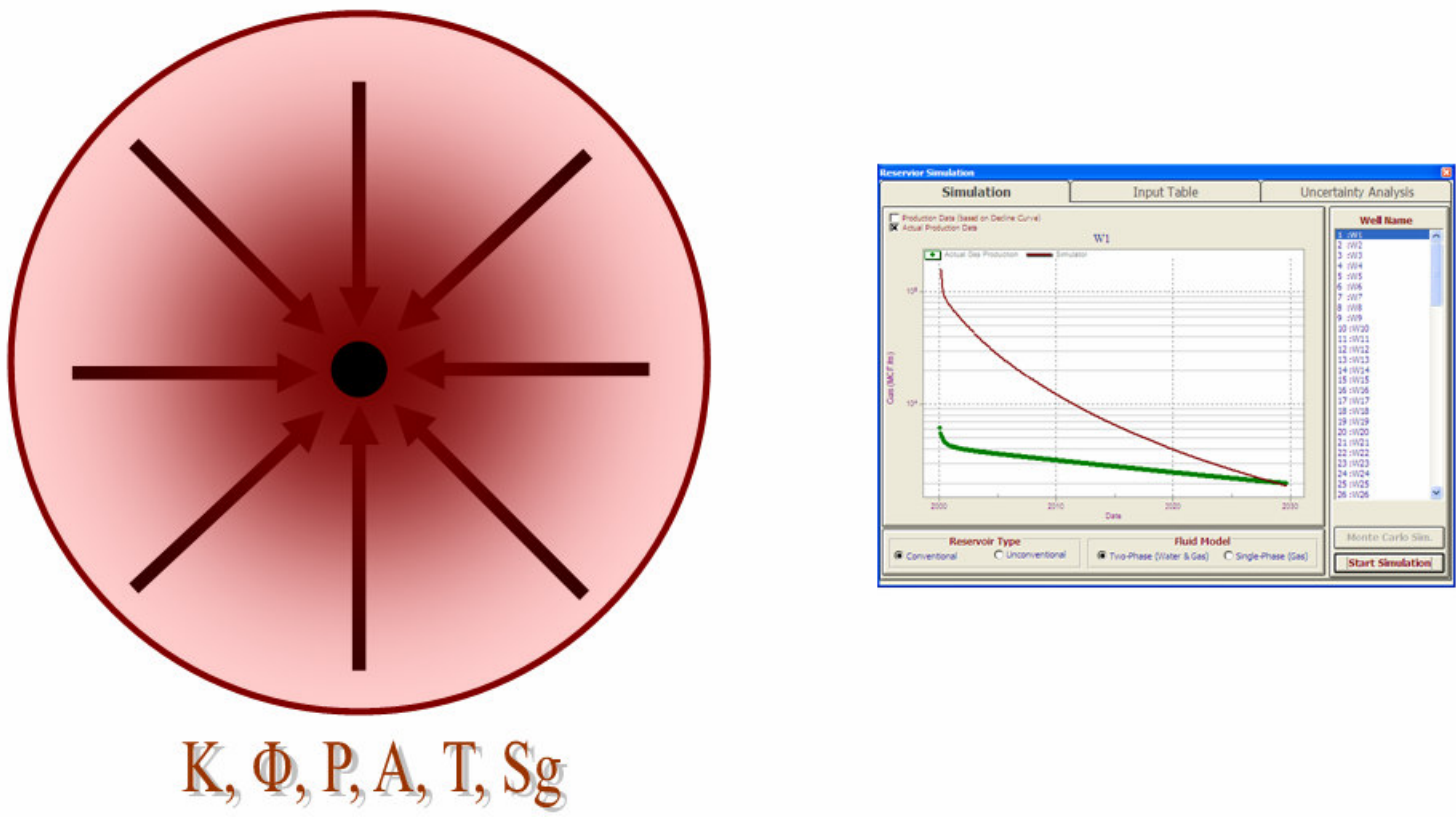

Fig. 3 - Principle of history matching.

The single-well radial simulation assumes radial flow in the lateral directions. The mathematical function that rules this process is the equation for fluid flow through porous media: 


$$
\frac{1}{r} \frac{\partial}{\partial r}\left(r \beta \frac{k}{\mu B} \frac{\partial P}{\partial r}\right)+\frac{\partial}{\partial z}\left(r \beta \frac{k}{\mu B} \frac{\partial P}{\partial z}\right)=\frac{1}{\alpha} \frac{\partial}{\partial t}\left(\frac{\phi}{B}\right)
$$

Where...

$\alpha=$ constant

$r=$ distance in radial direction in

cylindrical coordinates system
$B=$ formation volume factor

$k=$ permeability

$\beta=$ transmissibility conversion

factor $\mu=$ viscosity of the fluid

$\varnothing=$ porosity

The accuracy of this method depends on the quality of the reservoir model. However, there are thousands of combinations that will reproduce the behavior observed in the production data. Therefore, to converge into a unified result, it is necessary to integrate the history matching process with other techniques such as decline curve analysis and type curve matching. 


\subsection{Intelligent Production Data Analysis}

IPDA is a new methodology for analysis of production data that integrates well-known techniques applied for years in the industry (Decline Curve Analysis, Type Curve Matching and History Matching) with the arising technology of Intelligent Systems. The result is a unified set of reservoir characteristics based on a single input: monthly production rate data.

This innovative procedure removes the subjectivity associated with other techniques when used individually. It has proven to be quite useful for characterizing mature fields. The advantage of IPDA is the fact that records of monthly production rate data are required for conducting the analysis. However, in cases where other pieces of information are available (well logs, production intervals, pressure test periods, etc) it is recommended that they be included with the analysis. The process is simple and can be completed shortly (days, depending on the number of wells).

The methodology involves five steps:
1. Data Acquisition
2. Decline Curve Analysis
3. Type Curve Matching
4. History Matching
5. Field-Wide Pattern Recognition

Moreover, the IPDA technique provides different tools for evaluating actual and future behavior of individual wells.

One of the tools in the IPDA technique monitors the performance of wells in order to identify the underperformance wells. In many formations, it is common to find isolated wells that are not producing at full capacity. In most cases these 
wells are candidates for stimulation procedures. The IPDA technique has come up with a procedure for recognizing the under-performer wells.

An additional feature offered by this technique is the possibility of predicting the behavior of infill drilled wells. For a specific location, using this tool, the decline parameters (Qi, Di and b) may be estimated. As a result, the decline curve of the hypothetical well can be modeled.

The following is a detailed explanation of the tasks that have to be completed when performing an IPDA procedure.

\subsubsection{Data acquisition.}

In order to conduct an Intelligent Production Data Analysis, the information that contains the records of monthly production well data needs to be organized in a particular shape (See Table 1). The data import wizard supports text files (csv, txt, tab separated), Microsoft Excel files and Microsoft Access files. It is obligatory to have the following 5 pieces of information:

1. Well Name

2. Latitude

3. Longitude

4. Date of Production

5. Production Rate (MSCF/Month)

Other parameters, such as well logs, can be included when available. Obviously, while performing an IPDA, more information is included as the quality of the predictions increases. 


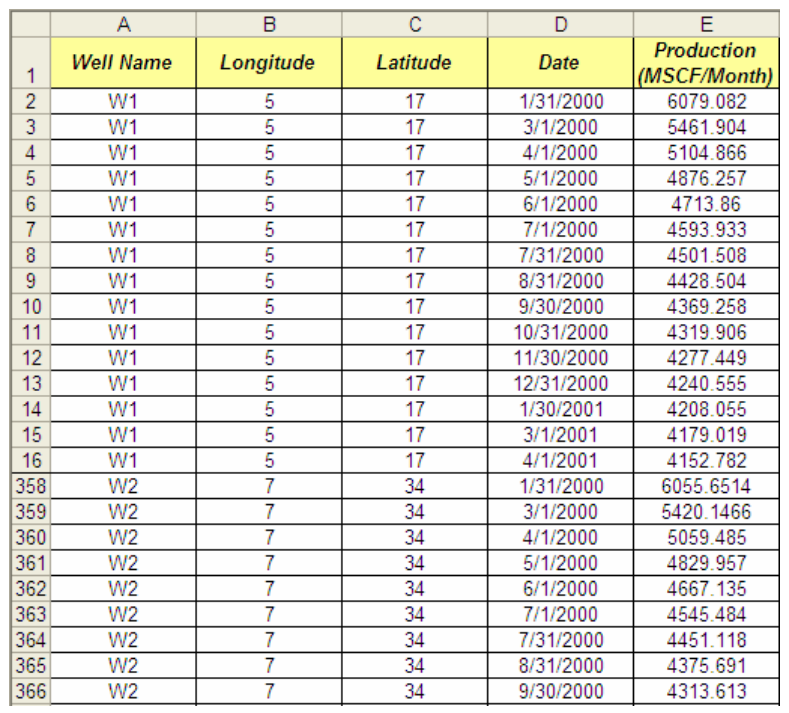

Table 1 - Production Data organized to be imported in IPDA.

\subsubsection{Decline Curve Analysis.}

After the data has been imported, the next step is performing a decline curve analysis for all the wells.

Both the original and the modeled data are plotted in a semi log scale. The decline parameters (Qi,Di and b) have to be adjusted until a satisfactory match has been accomplished. The most common type of plots employed for performing a decline curve analysis are Flow Rate vs. Time and Cumulative Production vs. Time (See Fig. 4).

In fig. 4, the green line represents a semi-log plot of Gas Flow Rate vs. Time while the blue line refers to Gas Cumulative Production vs. Time. First, it is necessary to select the desired type of decline curve: hyperbolic, harmonic or exponential (Fig. 4 - Red box).

The fitting process of the observed data takes place by modifying the values of Qi (Initial Flow Rate), Di (Initial Decline Rate) and b (Hyperbolic Exponent) until the desired curve is achieved (Fig. 4 - Orange Box). Finally, the $n$ number of years and the abandonment rate is introduced in order to calculate the Estimated Ultimate Recovery, EUR (Fig. 4 - Blue Box). 


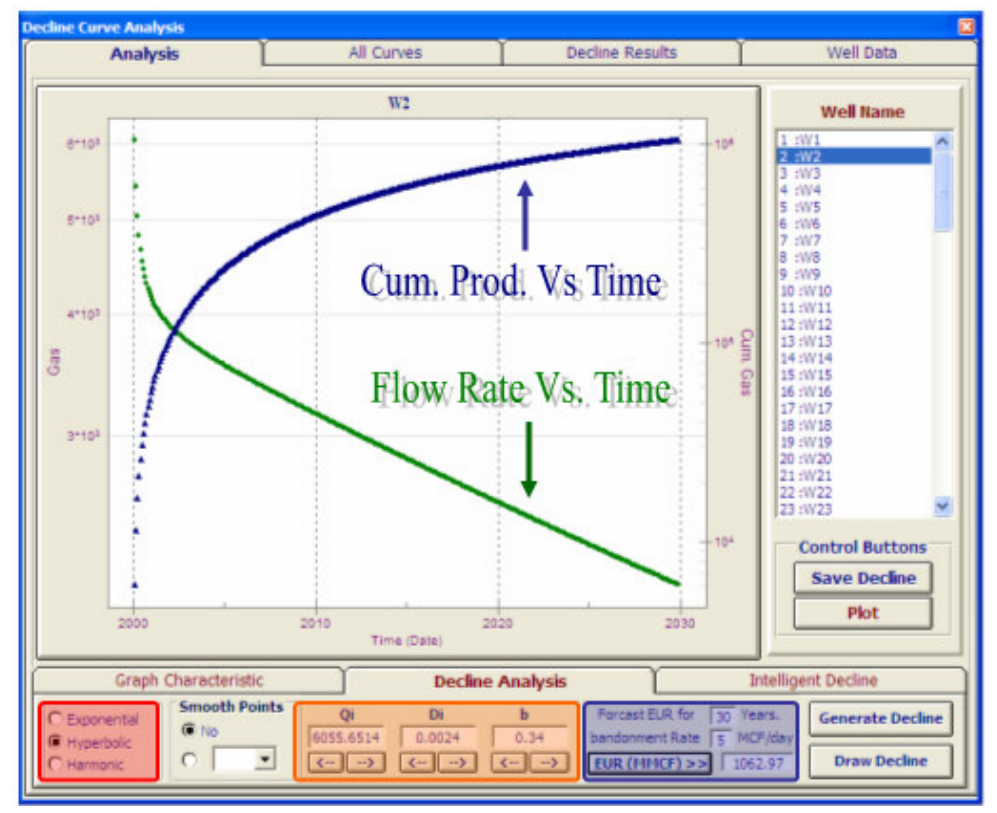

Fig. 4 - Initial stage of decline curve analysis.

Once a suitable match has been achieved and the Estimate Ultimate Recovery is calculated, it may be forecasted for number $n$ of years considering an abandonment rate of $x$ Mscf/month.

Finally, the decline curve information of each well is stored in a table.

\subsubsection{Type Curve Matching}

The IPDA uses Cox Approach for type curves. What distinguishes Cox Type Curves from Fetkovich Type Curves is the fact that under this analysis a reservoir engineer is able to perform rapid analysis of reservoir properties (kh), fracture effectiveness $(\mathrm{Xt})$, and well drainage area $(\mathrm{A})$ for fractured wells. The impact of skin on performance is considered, along with the effect of reservoir geometry (alternative length-to-width ratios).

For each well the hyperbolic exponent $b$ works as a link between decline curve analysis and type curve matching. 
The process starts when the $b$ value is imported from the stored information in the previous step (decline curve analysis) and a set of type curves is generated for that particular $b$. Then, the user selects the best fit according to other parameters such as: permeability $(K)$, fracture half-length $(X f)$ and drainage area $(A)$.

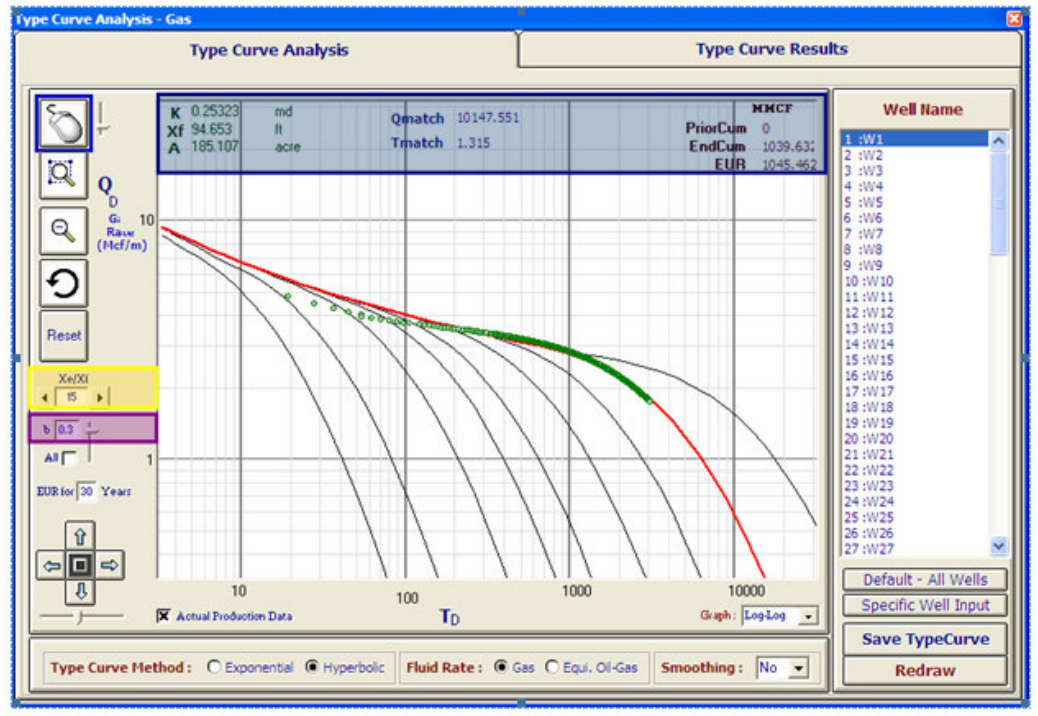

Fig. 5 - Type Curve Matching

In Fig. 5, the green dots represent the Production Data of the well. The $b$ value was imported from the stored information of the decline curve analysis (Fig. 5 - Purple box). Then, a set of type curves is generated for that specific $b$ value. After that, it is necessary to select the type curve that best fits the data. The next step is adjusting $\mathrm{Xe} / \mathrm{Xf}$ until the red line reaches the selected type curve (Fig. 5 yellow box). All the parameters calculated in this step will be available in the top part of the window (Fig. 5 -blue box).

Then the EUR is calculated, this time through type curve approach, a fairly different technique. 
Additional information is required during the type curve matching process. This information may be found in well logs or records of reservoir parameters (Initial Pressure, Initial Temperature, Isotropicity, etc).

The well parameters required are:

$\checkmark$ Porosity

$\checkmark$ Pay thickness

$\checkmark$ Gas saturation

$\checkmark$ Flowing bottom-hole pressure

The reservoir parameters needed are:

$\checkmark \quad$ Initial reservoir pressure

$\checkmark$ Average reservoir temperature

$\checkmark$ Gas specific gravity

$\checkmark$ Isotropicity

$\checkmark$ Drainage shape factor (L/W ratio)

Finally, the parameters calculated during the type curve matching process (EUR, $\mathrm{k}, \mathrm{A}, \mathrm{x}_{\mathrm{f}}$ ) are stored in a table. After completing this step, it is time for beginning the history matching.

\subsubsection{History Matching}

During the history matching process some parameters are imported from the type curve information. These values include Drainage Area and Permeability, which are calculated during the TCM process.

Importing variables from previous steps reduces considerably the subjectivity associated with Production Data Analysis. Three different methods 
are being used and they are inter-connected, which increases the chance of coming up with accurate estimations of reservoir parameters.

History Matching helps to benchmark the quality of the other two analyses. This process is accomplished through an iterative method that finishes when convergence of the three methods has been achieved.

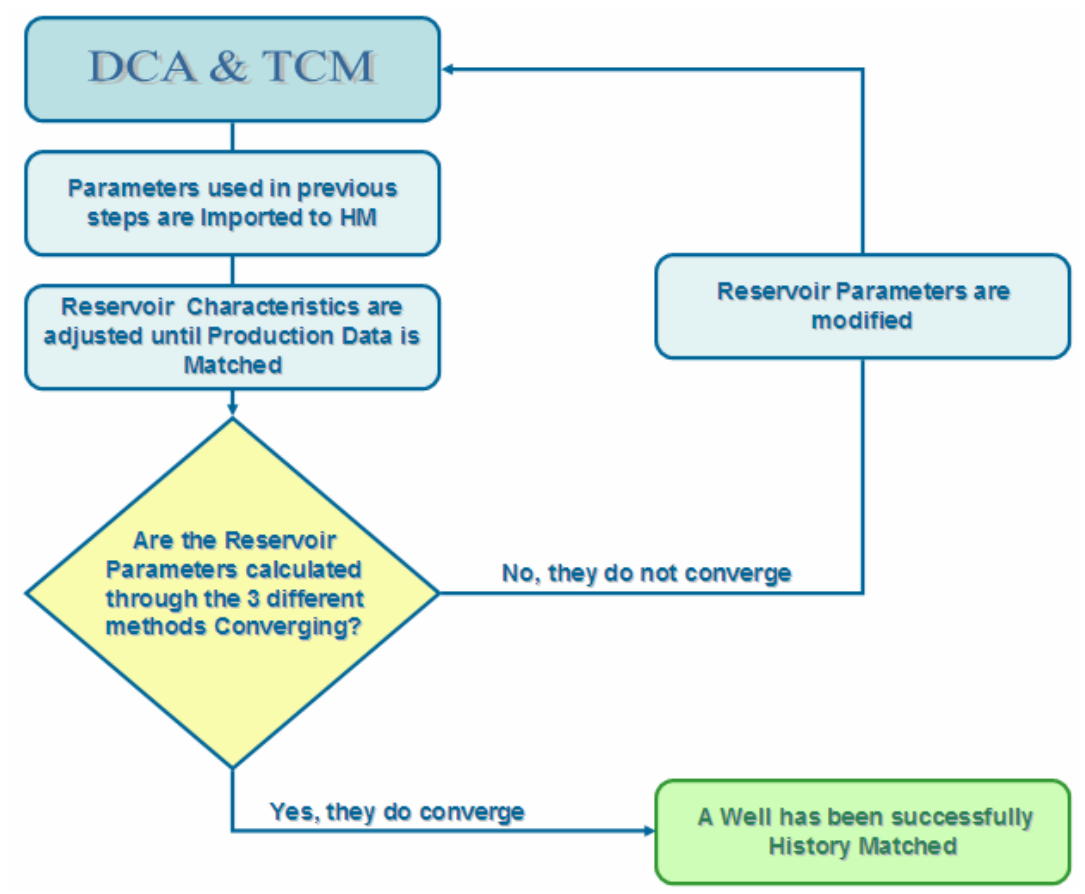

Fig. 6 - Flow chart of iterative process.

In fig. 6 the iterative process is explained. First, DCA and TCM are completed. Then, some of the reservoir properties, calculated in these steps, are employed to conduct the HM. The reservoir parameters need to be adjusted during the HM, but these modifications have to be updated in DCA and TCM. The process finishes when the same parameters are used for the three methods and the similar results are encountered. 


\subsubsection{Field-wide Pattern Recognition (FPR)}

Once the three techniques have been completed and the results have been optimized, the next step is the application of intelligent systems to analyze the data. This routine integrates the information obtained from all wells in order to predict Field-Wide behavior. FPR deduces a pattern in data that apparently does not register any relationship.

FPR gathers information about all the wells. Several production indicators can be evaluated. The variables that can be loaded in an RRQI map are:

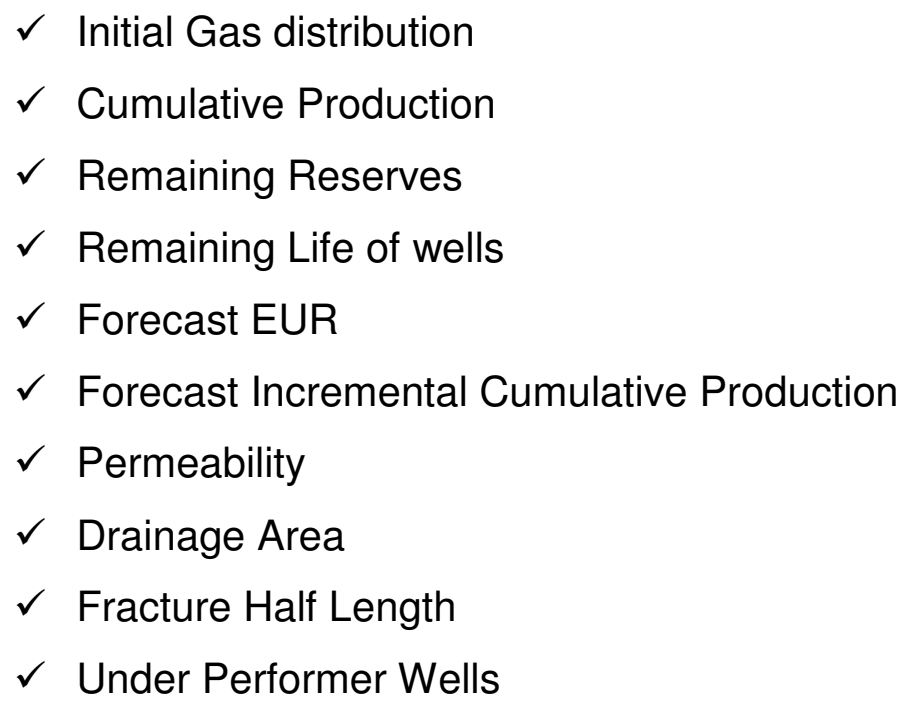

The plot shown in Fig. 7 is called Relative Reservoir Quality Index - RRQI. This is one of the maps offered by the IPDA technique to visualize results. The green box indicates the parameter under analysis. The cream boxes represent the two dimensions (latitude and longitude).

Each dimension is delineated in three zones employing the dashed lines (red line and blue line), the position of these lines can be adjusted using the controls on the red box. The zone above the red line is the "high zone," the area 
between the red line and the blue line is the "medium zone," and the area under the blue line is the "low zone."

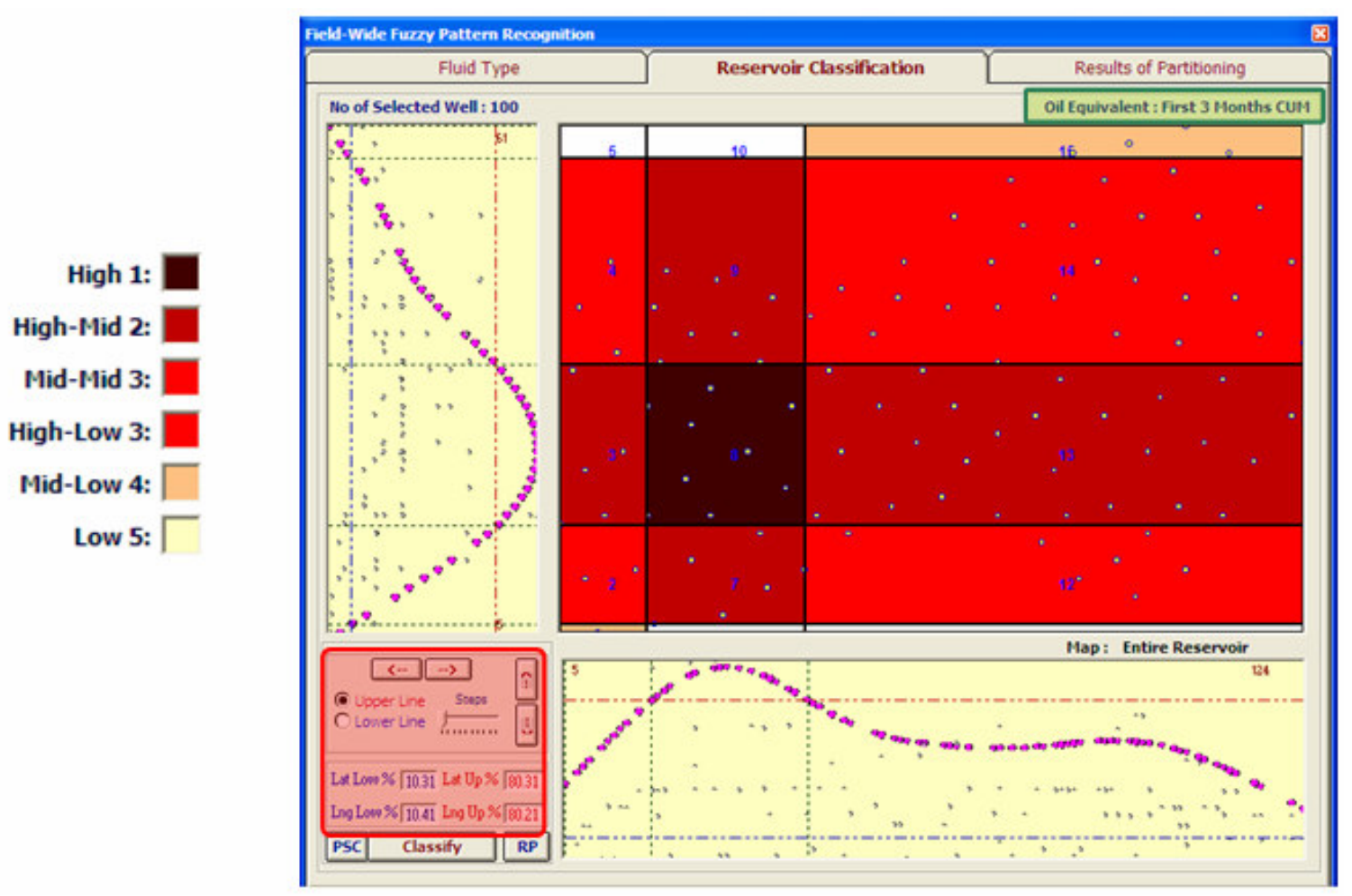

Fig. 7 - Relative Reservoir Quality Index RRQI.

The RRQI map is the result of projecting both dimensions in the same plane. As a result, five quality zones are created, each one with an assigned color (See legend in Fig. 7). The dark brown indicates the best quality zone and the light cream represents the lowest value for that specific indicator.

Also in Fig. 7 there is an important detail that needs to be mentioned. For each dimension box (cream boxes) there are two groups of dots. The gray dots represent the value that the indicator under evaluation (permeability, remaining reserves, initial gas distribution, etc.) has in each well. The purple dots represent the pattern deduced through the application of $F P R$. 
An additional way to visualize results in IPDA is the 3D-surface plot. This graph, shown in Fig. 8 consists of a tridimensional surface map of the parameter under evaluation. It can be rotated and observed from different positions. It has a colored legend that facilitates the identification of the regions with higher quality.

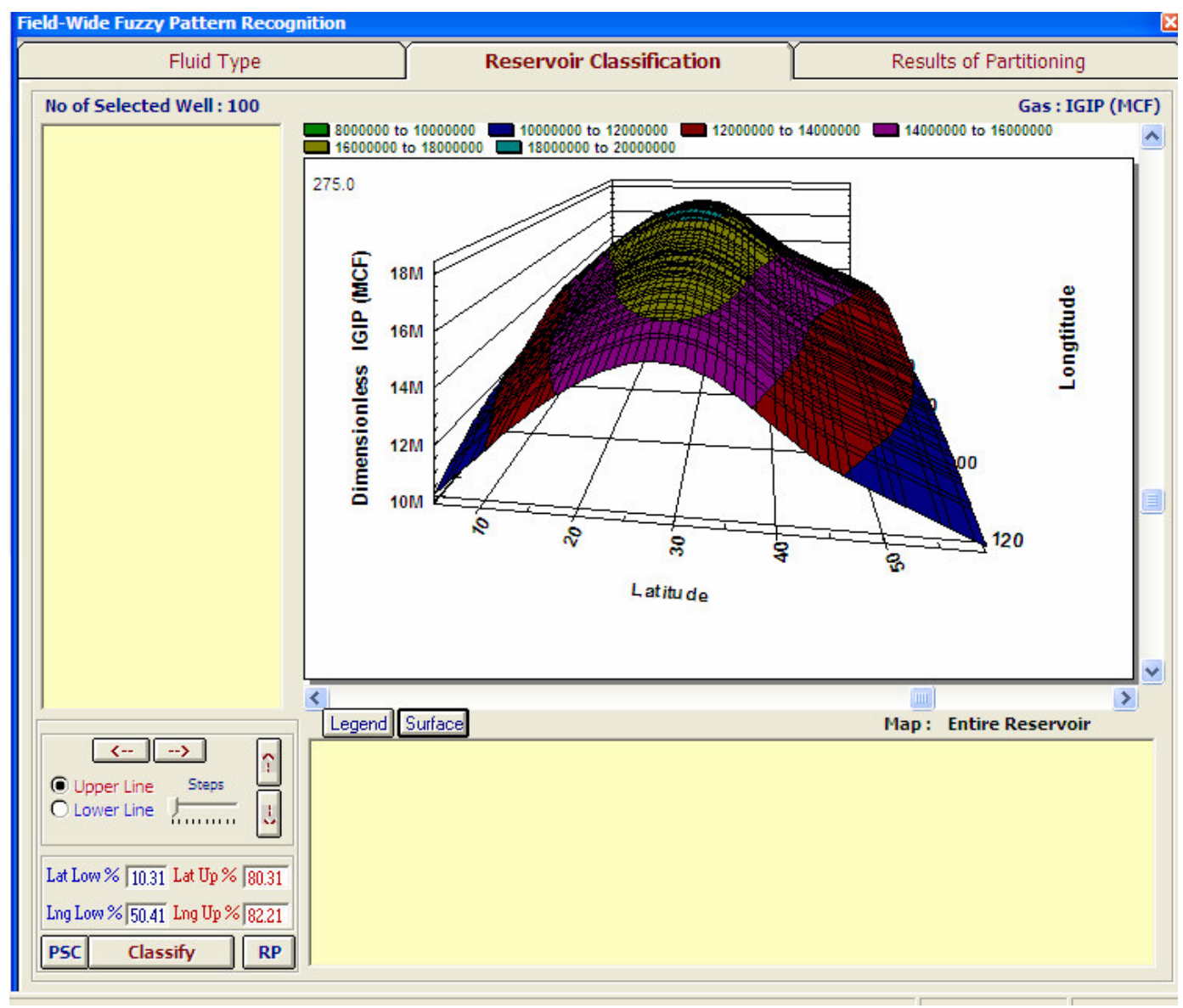

Fig. 8 - 3D-Surface plot.

At this point, all the steps that are required for conducting an IPDA analysis have been explained. Nevertheless, there is a set of tools offered by this methodology that may assist engineers in the evaluation of actual and future behavior of individual wells. One of these tools, named "Under-performer wells", monitors the productivity of the wells under analysis. 


\subsubsection{Under-performer wells.}

In many formations, it is common to find isolated wells that are not producing at full capacity. In most of the cases these wells are candidates for stimulation procedures. The IPDA technique has come up with a procedure for recognizing under-performer wells. The routine monitors the behavior of every well in the reservoir. It evaluates the performance of a well as compared to its neighbors in the surrounding region.

Fig. 9 explains the process in detail. Every zone in the reservoir (See colored legend) has an average value for the indicator under study. These values can be seen in a table that reports this information. A well will be labeled as an under-performer if it meets two conditions:

1. Its production is in the bottom $25 \%$ of its own zone.

2. Its production is below the average of the next zone.

Continuing with the example shown in Fig. 9, if a well is located in Zone 3, whose average value is 10 , and its individual production was measured to be 2 , this well would be labeled as an under performer, because it meets the two conditions already mentioned. First, the well is in the bottom $25 \%$ of its own zone, and its production is below the average of the next zone (zone 4). 


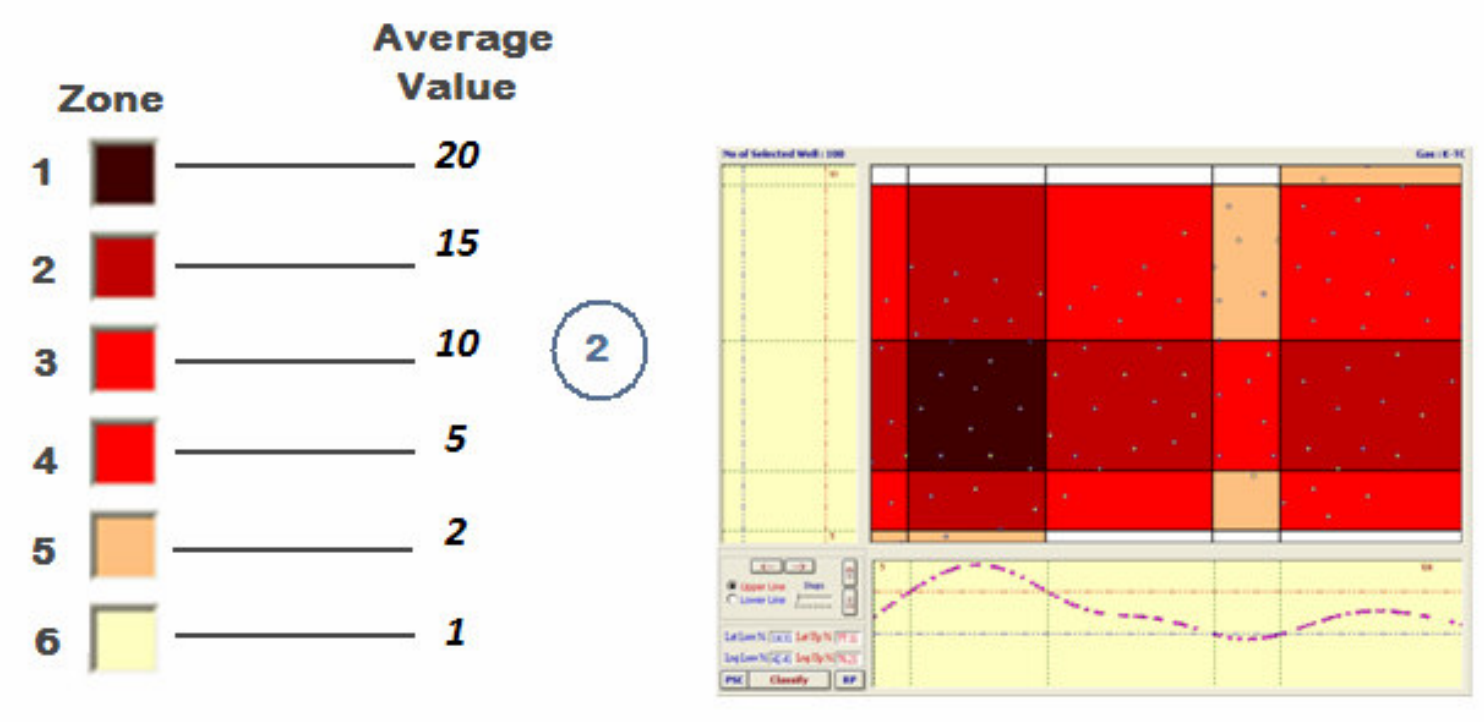

Fig. 9 - Under-Performer Wells.

In addition to the recognition of under-performers wells, the IPDA technique offers an additional feature that predicts the behavior of infill drilled wells. The tool is called "Evaluation of what-if scenarios" and it estimates the decline parameters $(Q i, D i$ and $b)$ at any location within the reservoir.

\subsubsection{Evaluation of what-if scenarios}

IPDA targets three main goals, forecasting remaining reserves, identifying sweet spots, and recommending locations for infill drilling. By comparing the different parameters obtained (i.e. remaining reserves, permeability, EUR, etc.) it is possible to recognize locations in the reservoir that are qualitatively superior and represent the best option for future drilling plans.

Engineers are obligated to support their recommendations in a comprehensive way. The best approach is always a solid economic analysis. 
The IPDA technique offers a tool called evaluation of what-if Scenarios. Using this feature, one can select a location in the reservoir and the decline parameters (Initial Production Rate " $Q_{i}$ ", Initial Decline Rate " $D_{i}$ " and Hyperbolic Exponent "b") will be calculated applying an intelligent procedure.

In Fig. 10 the blue dots are existing wells. The red dot indicates a location selected for infill drilling, which is the first step when using the "what-if scenarios" tool.

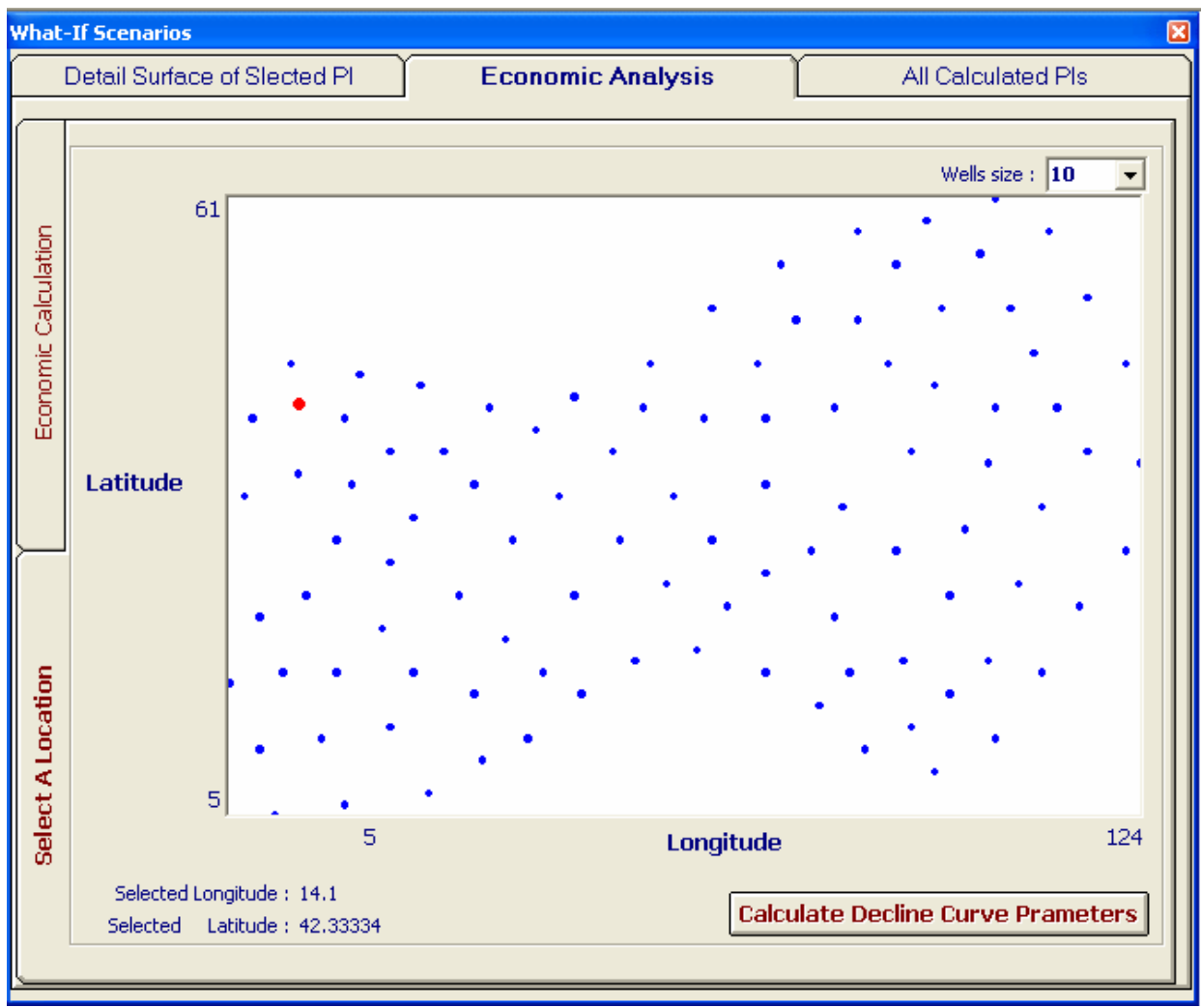

Fig. 10 - Location of a Future well.

After a location has been selected and the decline parameters have been calculated, it is necessary to create the curve and model the behavior of the well. Moreover, an economic analysis will be performed in order to assess the convenience of drilling the well in that specific zone. 
The Net Present Value ${ }^{11}$ (NPV) is a well-known indicator for financial appraisal of long-term projects. It measures the excess or shortfall of cash flows, in present value terms, once financing charges are met.

The equation for calculating NPV is:

$$
\mathrm{NPV}=\sum_{t=1}^{n} \frac{C_{t}}{(1+r)^{t}}-C_{0}
$$

Where...

$t$ - The time of the cash flow

$n$ - The total time of the project

$r$ - The discount rate

$C_{t}$ - The net cash flow (the amount of cash) at time $\mathrm{t}$

$C_{0}$ - The capital outlay at the beginning of the investment time $(t=0)$

Additional variables, such as Gas Price and years of production, are necessary in order to perform economic analysis.

Fig. 11 is a graphic representation of the production data forecasted for a well drilled in the location selected in Fig. 10. In this case, the economic analysis is performed for a time period of five years. 


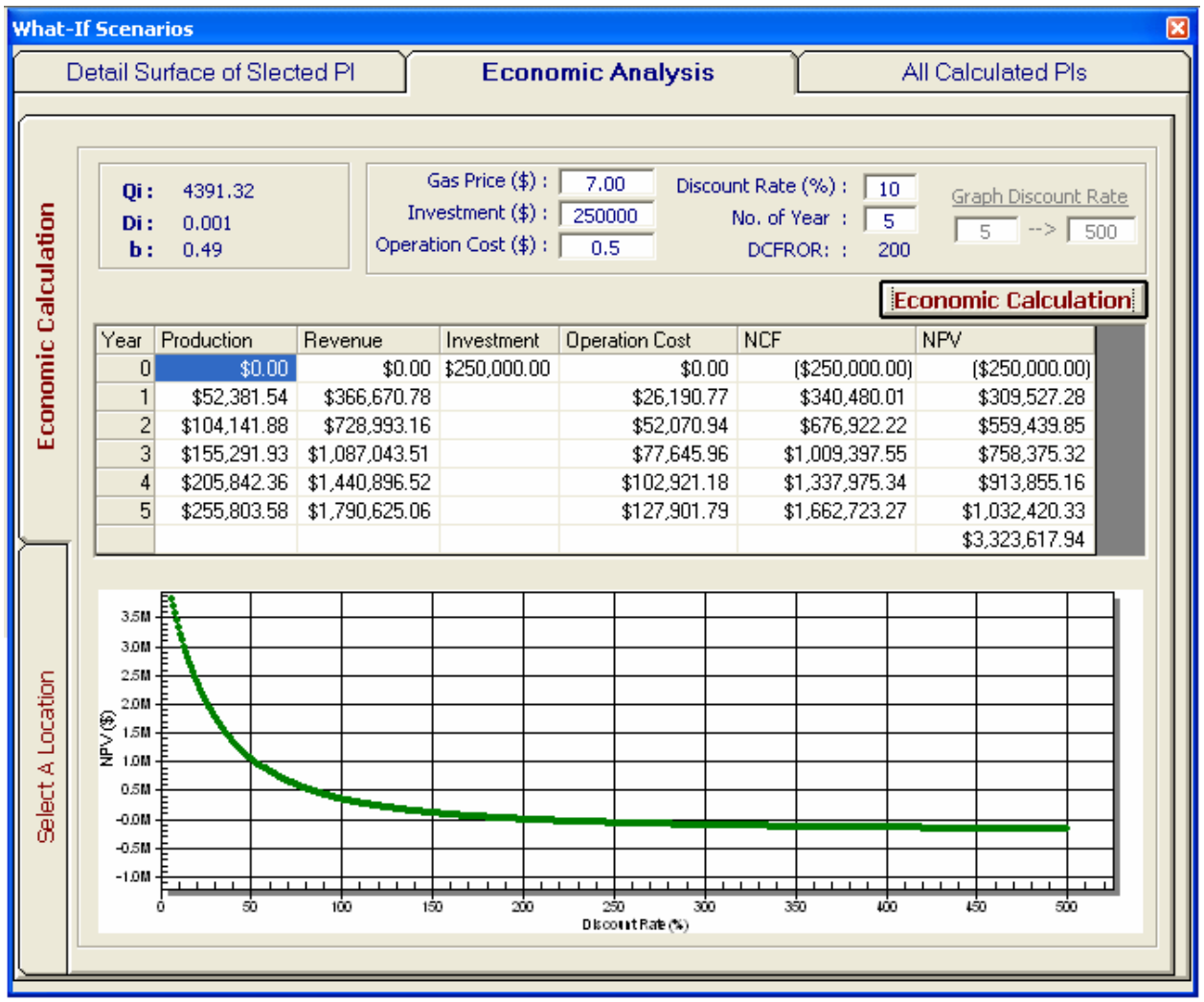

Fig. 11 - Predicted behavior of an infill drilled well. 


\section{Chapter III - Methodology}

The process of validation followed a routine specifically designed to evaluate the accuracy of IPDA technique. The procedure is shown in Fig. 12.

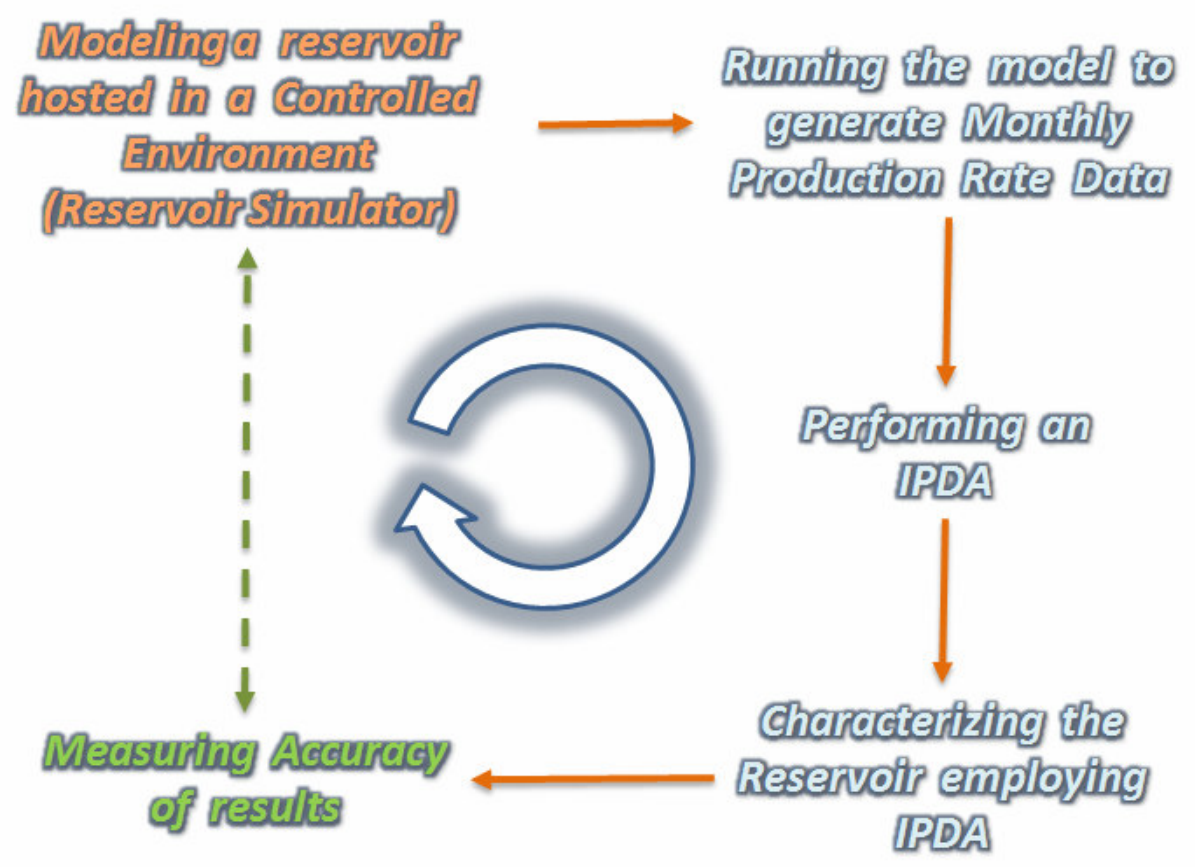

Fig. 12 - Flow-chart of the validation of IPDA technique.

Starting with the construction of a reservoir model in a controlled environment (reservoir simulator), each model was run and monthly production rate data was generated. Then, the records of production rate data were used as an input to perform an IPDA analysis. After that, different reservoir characteristics (initial gas distribution, remaining reserves, permeability distribution, etc.) were calculated through IPDA. Finally, the results were compared to the real situation (numerical simulator model) and the accuracy was verified.

Four variables were selected for comparing results and measuring accuracy of the IPDA technique. The following parameters were evaluated in each model studied: 
1. Initial Gas distribution.

2. Remaining Reserves Distribution at different times.

3. Estimated Ultimate Recovery (EUR).

4. Permeability Distribution.

Additional well properties that can be calculated using IPDA were also included in the verification of this technique. Specific situations were created in order to measure the ability of IPDA technique in estimating these variables. The well parameters studied in detail were:

$\checkmark \quad$ Fracture Half-Length, Xf.

$\checkmark$ Drainage Area, A.

$\checkmark$ Under-Performer Wells.

$\checkmark$ Behavior Infill drilled wells. 


\subsection{Modeling a reservoir in a controlled environment}

As it has been stated, the main objective of this study was validating the applicability of IPDA technique under different reservoir configurations. Therefore, it was necessary to perform the verification in a formation which properties and behavior could be rigorously monitored (a controlled environment). Hence, a commercial reservoir simulator (ECLIPSE) was employed to develop different reservoir models. The next step was deciding what type of formations should be studied.

The majority of the fields in the United States are low permeability reservoirs, so the study focused on those kinds of formations. An extended review took place in order to find a reservoir with a heterogeneous distribution of intrinsic properties. After that, a field located in Washington County, CO, was selected ${ }^{12}$.

The selected formation (Plum Bush Creek Field) consists of a single face (dry gas) reservoir with high levels of heterogeneity. The structural maps were available and each reservoir property (porosity, permeability, thickness, etc) was adapted to a uniform grid system. The grid system consists of a matrix of $128 \times 64 \times 4$ blocks so the reservoir can be divided in approximately 33,000 sectors. This partition was small enough to recreate an analogous distribution of the reservoir properties. Fig. 13 illustrates the grid system employed for modeling the field.

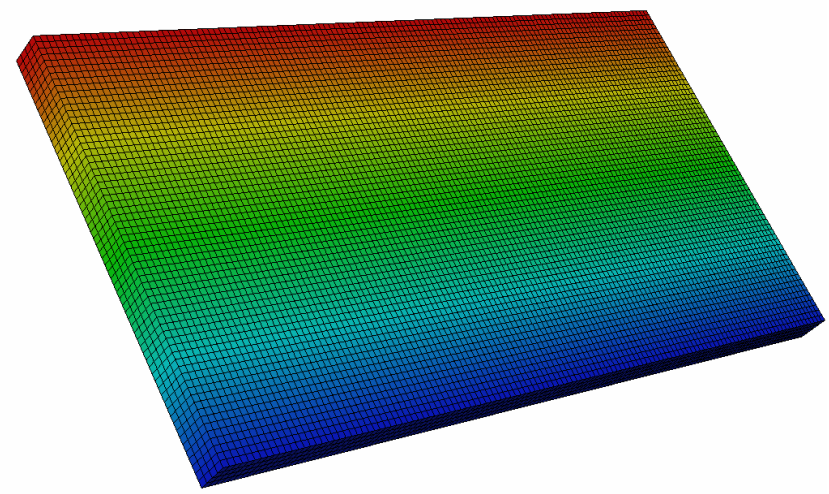

Fig. 13 - Grid-block Matrix. 
After the structural maps were partitioned and adapted to the grid-block system, the reservoir parameters were ready to be entered into the simulator. Two different kinds of formations were designed, a Single-layer and a Two-layer model. Both designs had heterogeneous distribution of intrinsic properties (porosity, permeability and thickness). Some of the reservoir characteristics were similar. In both cases, they had a single face composition (gas), located at a depth of $5000 \mathrm{ft}$. For both formations the overall size was about 10,000 acres and the initial pressure was set to be 3000 psi.

\subsubsection{Single-Layer Model}

The single layer model was an excellent candidate for testing the accuracy of the IPDA predictions. This reservoir was inspired by the aforementioned Plum Bush Creek Field, in Washington County, CO. The characteristics of this field are so diverse that even with all the property maps, it is still difficult to predict which zone in the reservoir has the higher concentration of hydrocarbon. (All the maps for $\mathbf{S} \boldsymbol{M} \boldsymbol{M}$ are available in Appendix A.)

Table-2 summarizes the properties of the Single-Layer Model (SLM). In this case, the thickness distribution is heterogeneous and is ranged between 75 and 300 $\mathrm{ft}$. The permeability was set within an interval of 0.5 and $3 \mathrm{mD}$, having three spots with high permeability in the reservoir (Isopermeability Map available in Appendix A). The isotropicity, which is the ratio of Kx over Ky, was equal to 1. Finally, the porosity was distributed from 8 to $12 \%$ throughout the field. 


\section{Table 2 - Characteristics of Single-Layer Model}

Composition: Dry Gas

Grid System: 128 × 64 × 3

Size: 10,000 Acres

Depth: $5000 \mathrm{ft}$

Initial Pressure: 3000 psi.

Thickness: Heterogeneous from 75 to $300 \mathrm{ft}$..

Appendix A

Porosity: Heterogeneous from $8 \%$ to $12 \%$

Permeability: Heterogeneous from 0.5 to $3 \mathrm{mD}$

Isotropicity: $K_{y}=K_{x} ; K_{z}=0.1 K_{x}$

Two cases of the Single Layer Model were studied. In this case, the objective was evaluating how the location of the wells affected the accuracy of IPDA analysis. Therefore, each version had a different well distribution.

The first version was called SLM-224. This adaptation of the single layer model had 224 wells cluster-distributed throughout the reservoir (See Fig. 14).

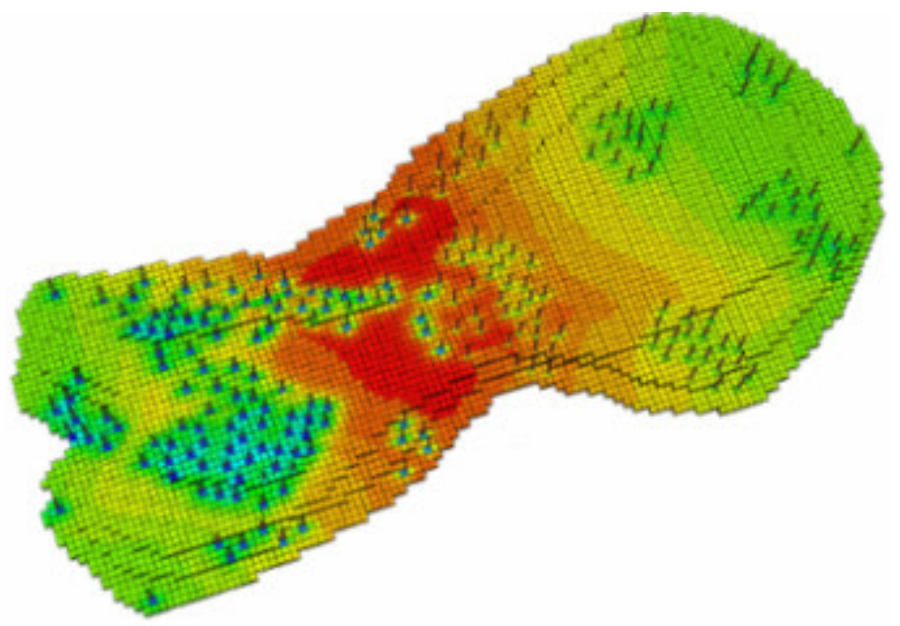

Fig. 14 - SLM 224 - Well distribution. 
The second version, named SLM-100, had 100 wells uniformly distributed over the whole field (See Fig. 21).

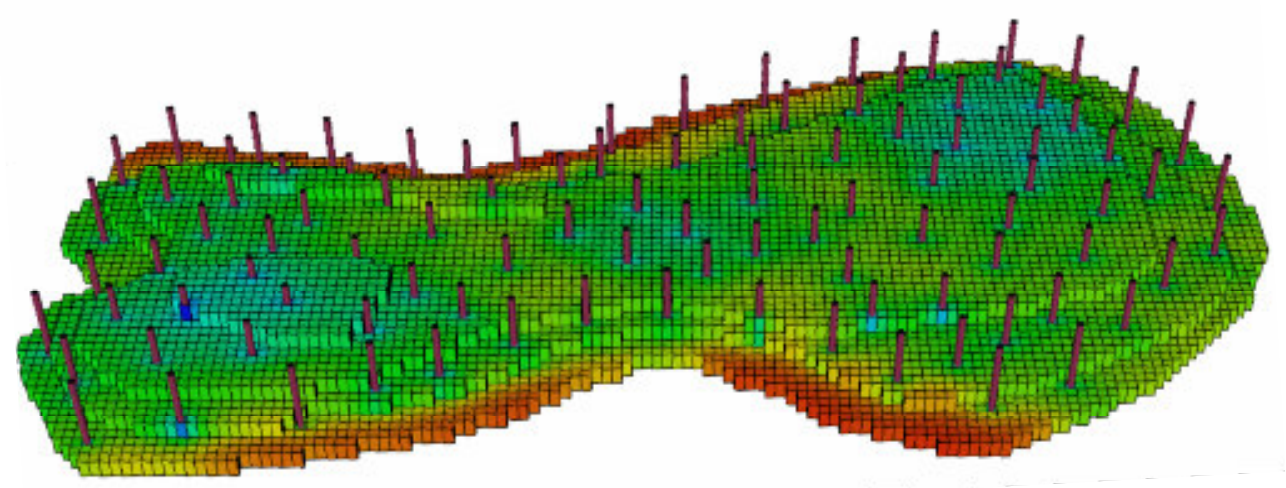

Fig. 15 - SLM 100 - Well distribution.

Again, these two versions of $S L M$ were created in order to examine the impact of well location over the predictions obtained through IPDA technique. Furthermore, it was necessary to determine if there was a relationship between the number of wells included in the analysis and the quality of results.

\subsubsection{Two-Layer Model}

The two-layer model was developed in order to examine the performance of IPDA in multi-layer formations. This reservoir consists of two isolated layers, each 75 ft. thick, and separated by $75 \mathrm{ft}$. Each layer had a unique distribution of reservoir properties, meaning that for every reservoir property the top layer had a different distribution than the bottom layer.

The premise, to be proved using the $T L M$, was that regardless of the characteristic of formation (properties distribution, multiple pay zones, wells distribution), the IPDA technique is capable of modeling an equivalent reservoir that behaves similar to the field under evaluation. 
Table 3 summarizes the properties of the Two-Layer Model (TLM). The permeability was heterogeneously distributed within an interval of 1 and $5 \mathrm{mD}$, having spots with high permeability in each layer. Finally, the porosity was distributed from 8 to $12 \%$ in the bottom layer and 13 to $25 \%$ in the top layer. (All the maps for $\mathbf{T L M}$ are available in Appendix B.)

\section{Table 3 - Characteristics of Two-Layer Model}

\section{Characteristics}

All Maps Available at

Composition: Dry Gas

ఏ)

Grid System: 128 × 64 × 3

Size: 10,000 Acres

Depth: $5000 \mathrm{ft}$

Initial Pressure: 3000 psi.

Thickness: 75 each layer.

Appendix B

Layer Separation: 75 feet

Porosity: Heterogeneous from $8 \%$ to $25 \%$

Permeability: Heterogeneous from 1 to $5 \mathrm{mD}$.

Isotropicity: $K_{y}=K_{x} ; K_{z}=0.1 K_{x}$

For the Two-Layer Model three versions were created. In this case, the objective was evaluating the ability of the IPDA technique to recognize intrinsic properties in a reservoir. Therefore, for each version a property of the formation (either permeability or porosity) was set to be homogeneous and the other parameter was heterogeneously distributed over the field.

For example, version TLM-1 had homogeneous porosity and heterogeneous permeability. TLM-2 had homogeneous permeability but heterogeneous porosity. Finally, TLM-3 had both porosity and permeability heterogeneously distributed over the reservoir. In these three cases, it was possible to evaluate the capability of the IPDA technique in recognizing intrinsic properties at a field-wide level. 
The three versions had 100 wells uniformly distributed throughout the reservoir (See Fig. 16).

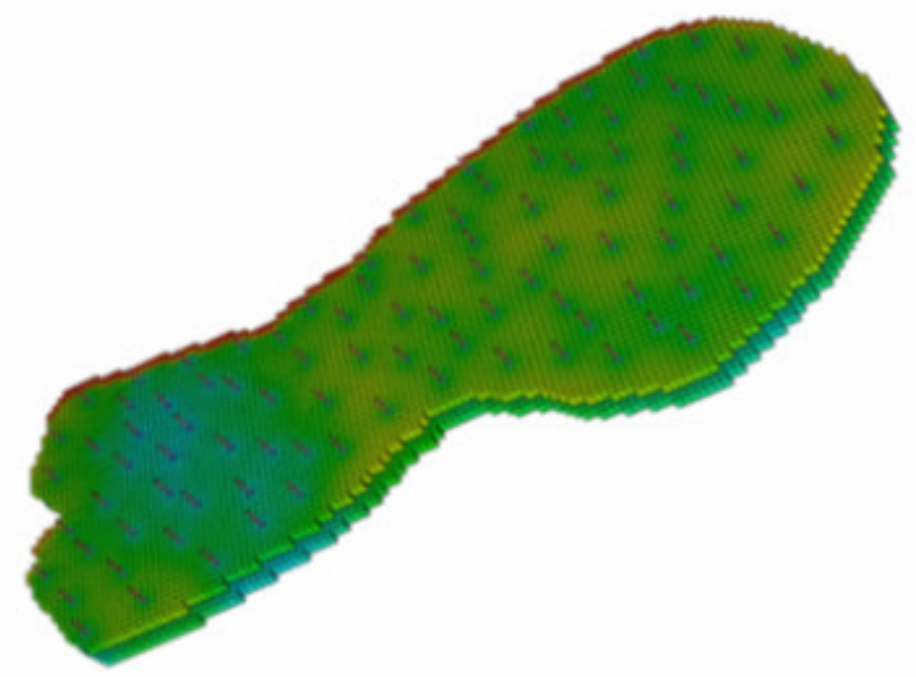

Fig. 16 - Well distribution of Two-Layer Models.

In conclusion, five different reservoirs (two Single-Layer and three Two-Layer) were developed in order to verify the accuracy of the IPDA technique. Each version was created specifically to evaluate the capacity of the IPDA to recognize diverse conditions. Next, it was necessary to determine a production strategy for each formation (See Table 4).

\begin{tabular}{ccccc}
\hline \multicolumn{5}{c}{ Table 4 - Reservoir Versions } \\
Model & $\begin{array}{c}\text { Porosity } \\
\text { Distribution }\end{array}$ & $\begin{array}{c}\text { Permeability } \\
\text { Distribution }\end{array}$ & $\begin{array}{c}\text { Number of } \\
\text { Layers }\end{array}$ & $\begin{array}{c}\text { Number } \\
\text { of Wells }\end{array}$ \\
\hline SLM-224 & Heterogeneous & Heterogeneous & 1 & 224 \\
SLM-100 & Heterogeneous & Heterogeneous & 1 & 100 \\
TLM-1 & Homogeneous & Heterogeneous & 2 & 100 \\
TLM-2 & Heterogeneous & Homogeneous & 2 & 100 \\
TLM-3 & Heterogeneous & Heterogeneous & 2 & 100 \\
\hline
\end{tabular}




\subsection{Schedule of Production}

Once each reservoir was modeled in the simulator, a production strategy was necessary to generate monthly production rate data, so these records could be entered into the IPDA.

Many variables such as type of completion, well diameter, production control mode (constant rate, constant pressure), drilling schedule, etc. were required. Therefore, a literature review of production in mature fields was performed for the purpose of identifying the common practices in this type of formation. It was found that the majority of the wells in mature fields have an open-hole completion. It is also common for the production in these wells to be controlled by constant pressure (50 to $100 \mathrm{psi})$.

The findings showed that vertical wells should be drilled with the following characteristics:

$\checkmark$ Diameter of $0.5 \mathrm{ft}$

$\checkmark$ Open-hole completion.

$\checkmark$ Producing at constant Bottom-Hole Pressure of 100 psi.

$\checkmark$ Initially without skin.

As it was mentioned before, five different reservoir versions were modeled in order to validate the IPDA technique. Four of these versions (SLM-100, TLM-1, TLM-2, and TLM-3) have 100 wells (Fig. 15 and Fig. 16). The other version (SLM224) has 224 wells (Fig. 14). The next step was to design a drilling schedule for perforating the wells.

In both cases (SLM and TLM) the wells were drilled in groups of ten, with intervals of three months between each group. Therefore, drilling the 100 wells took about two and a half years (See Table - 5). 


\begin{tabular}{|c|c|c|}
\hline \multicolumn{3}{|c|}{ Table 5 - Drilling Schedule for 100 wells } \\
\hline Groun & Wells & Starting Operation \\
\hline Give & & Date \\
\hline 1 & W1, W2, W3, W4, W5, W6, W7, W8, W9, W10 & Jan 12000 \\
\hline 2 & W11, W12, W13, W14, W15, W16, W17, W18, W19, W20 & Apr 12000 \\
\hline 3 & W21, W22, W23, W24, W25, W26, W27, W28, W29, W30 & Jul 12000 \\
\hline 4 & W31, W32, W33, W34, W35, W36, W37, W38, W39, W40 & Oct1 2000 \\
\hline 5 & W41, W42, W43, W44, W45, W46, W47, W48, W49, W50 & Jan 12001 \\
\hline 6 & W51, W52, W53, W54, W55, W56, W57, W58, W59, W60 & Apr 12001 \\
\hline$\cdot$ & $\cdot$ & $\cdot$ \\
\hline$\cdot$ & $\cdot$ & $\cdot$ \\
\hline$\cdot$ & $\cdot$ & . \\
\hline 10 & W91, W92, W93, W94, W95, W96, W97, W98, W99, W100 & Jul 12003 \\
\hline
\end{tabular}

At this point the reservoirs were modeled and hosted in the simulator. The wells were designed, drilled, and began producing. The next step was determining the amount of data that should be generated and how long the wells should produce in each model.

The IPDA technique can be performed at any time in the life of a field. However, better results can be obtained once the pseudo-steady state has been achieved. Essentially this occurs due to the fact that production analysis techniques (decline curve analysis, type curve matching and history matching) provide better results once the transient period has passed.

The wells drilled had a minimum of 40 acres spacing. As a result, it took several years to reach the pseudo-steady condition. The two versions of the Single Layer Model (SLM-224 and SLM-100) produced for 30 years. On the other hand, the three versions of the Two-Layer Model produced for 26 years. 
In both cases, records of monthly production rate data were stored, so they could be used in the next step of the validation process, which was performing the IPDA analysis.

Fig. 17 is a plot of Production Rate vs. Time for six different wells in the model SLM (version SLM-100). The six wells are neighbor wells, located very close to each other. They performed quite differently, however, as is obvious, given the results shown in fig. 17. These results demonstrate the validity of the IPDA Technique by showing the degree to which the selected formation was heterogeneous.

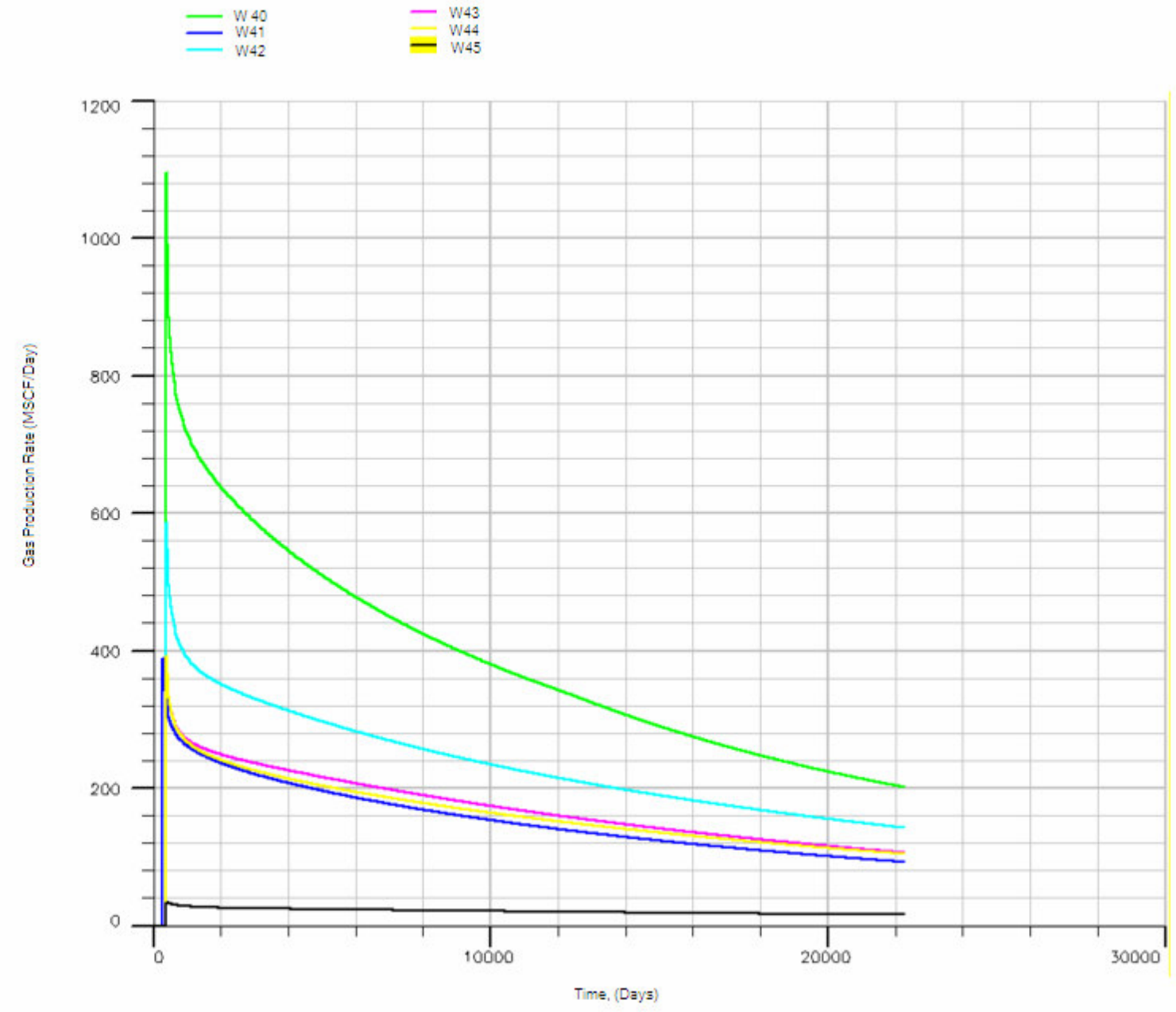

Fig. 17 - SLM-100. Production Rate vs. Time in neighbor wells 
Thus far, the following activities had been accomplished:

$\checkmark$ Five different versions were modeled and hosted in the simulator (controlled environment).

$\checkmark$ For each model, group of wells were drilled taking intervals and produced for a certain period of time.

$\checkmark$ Records of monthly production Rate data were generated and stored.

For each one of the five versions, the generated records of monthly production rate data were taken, the IPDA analysis was performed, and the characteristics of each reservoir were determined. 


\subsection{Performing the IPDA Analysis.}

Performing an IPDA analysis required five variables:
$\checkmark$ Well Name
$\checkmark$ Latitude
$\checkmark$ Longitude
$\checkmark$ Gas Production Rate (Mscf/month)
$\checkmark$ Date $(\mathrm{mm} / \mathrm{dd} / \mathrm{yy})$

Also, other parameters were necessary to conduct the analysis:
$\checkmark$ Initial Reservoir Pressure
$\checkmark$ Average Reservoir Temperature
$\checkmark$ Gas Specific Gravity
$\checkmark$ Isotropicity (Kx - Ky ratio)
$\checkmark$ Drainage Shape Factor
$\checkmark$ Average Porosity
$\checkmark$ Average Pay Thickness
$\checkmark$ Average Gas Saturation
$\checkmark$ Average Bottom Hole Pressure

The records of monthly production rate data were received from the simulator in a format that was not compatible with the IPDA. Considering the immense amount of information, a short code was necessary to facilitate the organization of the data. (Appendix $C$ contains the code that was developed for this task).

After the Production Data was collected and organized in the appropriate format, the IPDA analysis was initiated. The first step was performing decline curve analysis for each well. After that, the type curve matching process took place, also 
on a well-to-well basis. Once the type curve process was finished, the history matching was achieved.

The three previously mentioned procedures were accomplished through an iterative process (while performing one task, the other two were monitored). This reduced the subjectivity associated with these methods when they were applied individually.

\subsubsection{Decline Curve Analysis}

Fig. 18 describes the procedure of decline curve analysis applied in one of the wells under evaluation. The graphs shown represent a semi-log plot of Gas Flow Rate vs. Time. In both pictures, the green line is the actual data (simulator) and the blue line is the desired decline. The left picture illustrates the initial step. Then, by adjusting the decline parameters (Qi, Di and b) the curve was matched. The right picture shows the final results once the curve was corrected.
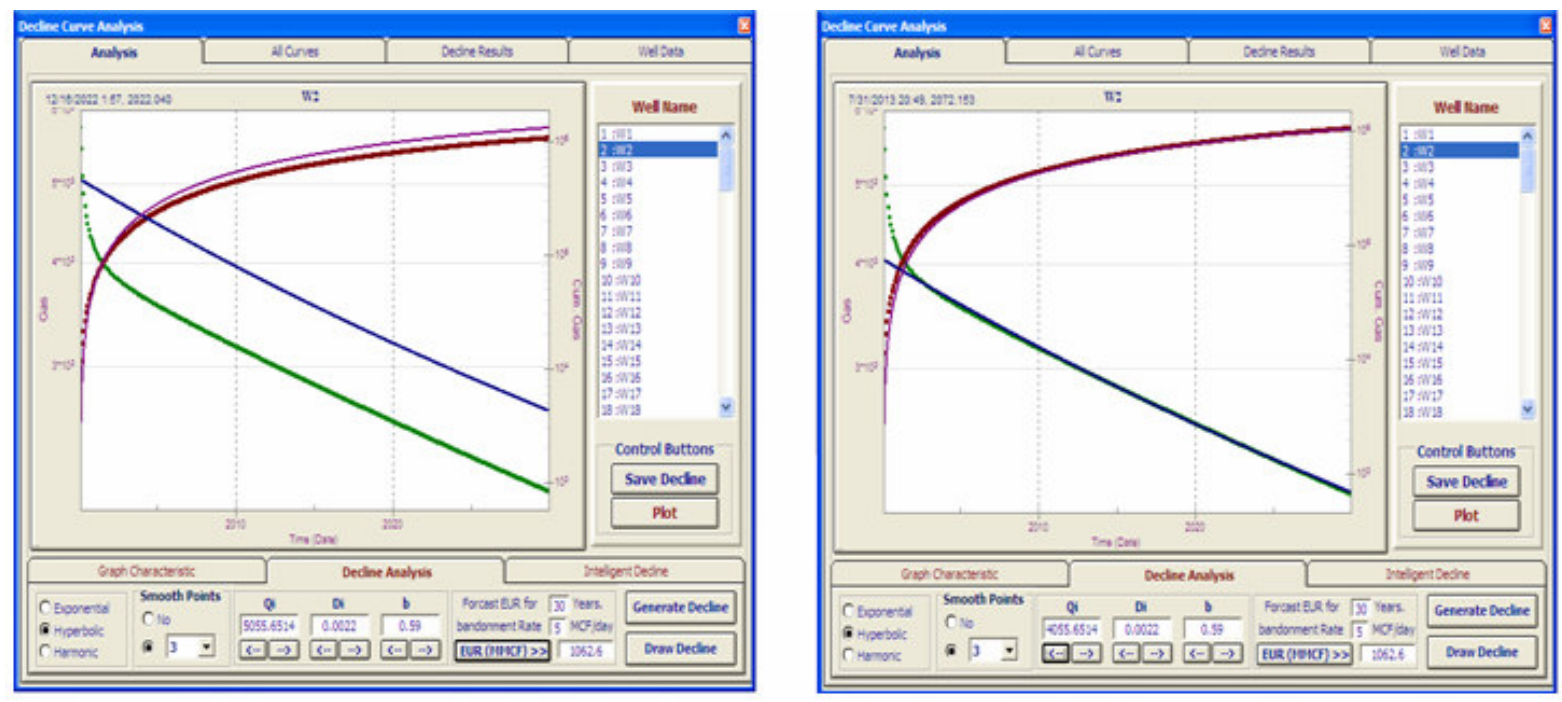

Fig. 18 - Final match in a decline curve analysis. 


\subsubsection{Type Curve Matching}

Since the reservoirs modeled represented a low permeability gas reservoir, it was opportune to use the Cox approach, because this type of curve is designed specifically for low permeability gas reservoirs with hydraulically fractured wells.

As noted above, the IPDA technique uses an iterative process to reduce the subjectivity of conventional techniques. The same variables (EUR, permeability, drainage area, etc.) are being calculated using three different techniques. Therefore, it is necessary to monitor the results achieved in each step to make sure that they agree with the values obtained in the previous stages of the analysis.

For example, the EUR is one of the variables employed for monitoring the convergence of decline curve analysis and type curve matching. It is important to verify that the EUR obtained during the type curve matching is similar to the EUR calculated through decline curve analysis. If a significant difference is found (between the EUR estimated from Decline Curve Analysis (DCA) and Type Curve Matching (TCM)), one easily realizes this incompatibility. Many times this issue simply requires going back to DCA to perform this process again.

This contrast permitted the identification of those wells that had inconsistent data, which were isolated and excluded from further steps in the analysis.

\subsubsection{History Matching}

In this step, a single-well radial simulator was used to match observed records of production data for each well.

In order to connect this process with the previous steps of the analysis (decline curve analysis and type curve matching) the permeability and the drainage 
area (calculated during previous phases) were imported and employed with other parameters for performing the history matching.

In Fig. 19, the green boxes represent the values imported from previous steps. The boxes highlighted in orange indicate the required information for initiating the process of history matching.

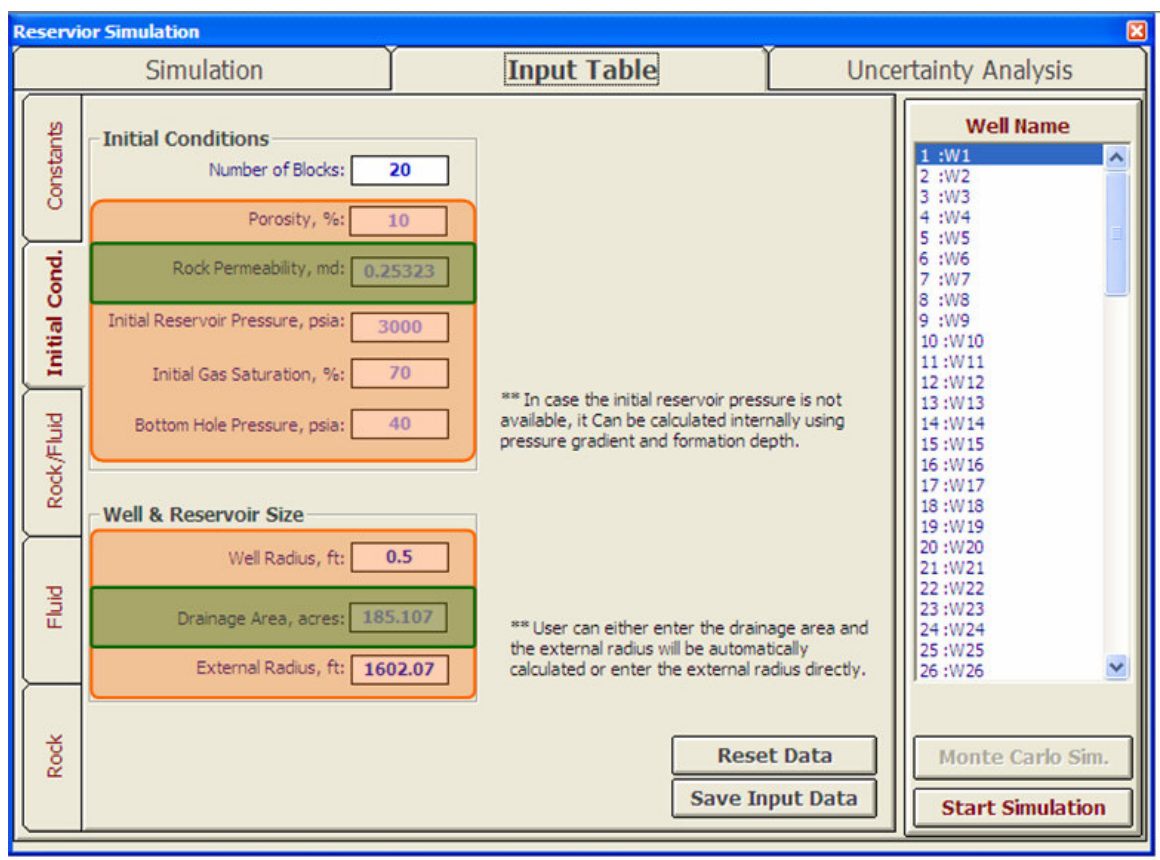

Fig. 19 - Initial Conditions for History Matching.

Since History Matching was the last step of the conventional analysis, it was a good opportunity for optimizing the quality of the evaluations.

When a reservoir parameter was modified during the History Matching process, these modifications were adjusted in DCA and TCM. The iterative process was finished when the same parameters were used for the three methods and the results were converged in an integrated value.

Fig. 20 is an example of a well which was successfully history matched. The green box indicates the EUR value. 


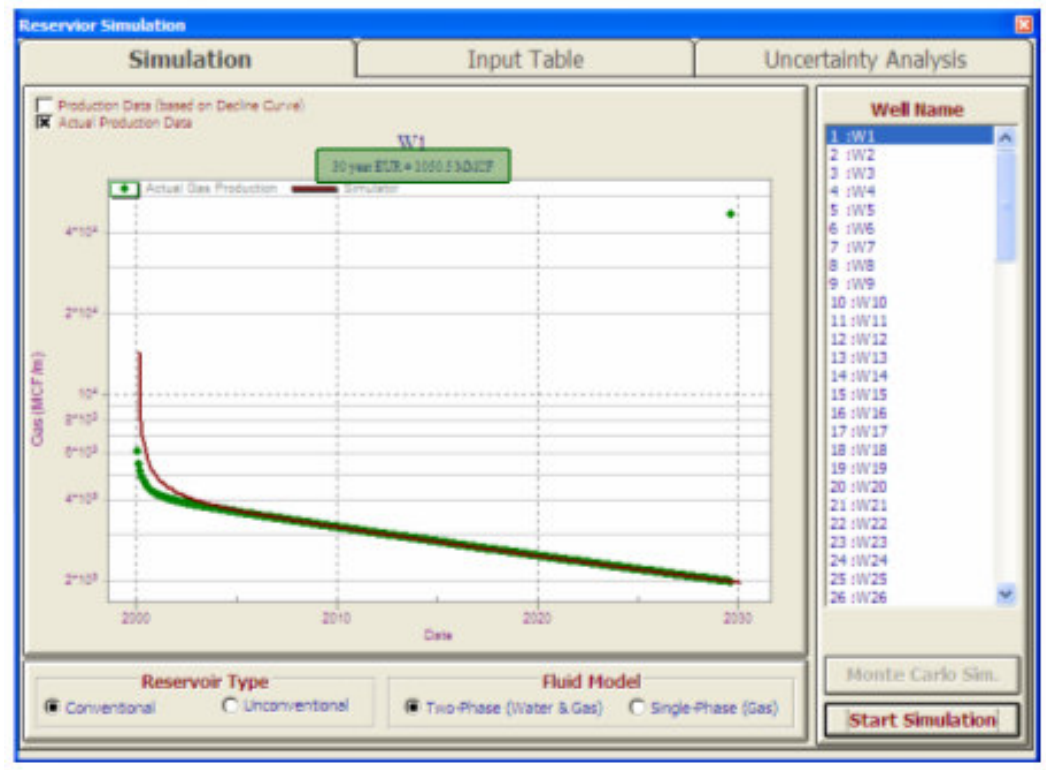

Fig. 20 - Successful History Match.

Once the three procedures ( $D C A, T C M$ and $H M$ ) were completed, a checking routine was performed in order to detect any inconsistencies in the data. The optimization process was necessary to ensure that the best possible analysis was achieved. The EUR values from DCA, TCM and HM were compared. When a significant difference (more than 10\%) was encountered, the calculations for that particular well were reviewed and performed again. This filtered out the wells that should not be considered for the rest of the analysis.

\subsubsection{Field-wide Pattern Recognition.}

The field-wide pattern recognition $(F P R)$ was performed once the conventional analysis of the production data was completed. FPR offered three categories of indicators: the first type, for evaluating the performance and productivity of the wells, the second, for forecasting reserves, and the third, for providing reservoir intrinsic properties.

The indicators for monitoring well performance are: 
$\checkmark$ Best 3 Months of production

$\checkmark$ Best 6 Months of production

$\checkmark$ Best 9 Months of production

$\checkmark$ Best 12 Months of production

$\checkmark$ First 3 Months of production

$\checkmark$ First 6 Months of production $\checkmark$ First 9 Months of production

$\checkmark$ First year cumulative production

$\checkmark$ Three year cumulative production

$\checkmark$ Five year cumulative production

$\checkmark$ Ten year cumulative production

The indicators for forecasting reserves are:

$\checkmark$ Initial Gas Distribution

$\checkmark$ Forecast Incremental Production

$\checkmark$ Estimated Ultimate Recovery

$\checkmark$ Remaining Reserves Distribution

$\checkmark$ Remaining Life of wells

The indicators for characterizing reservoir properties are:

$\checkmark$ Permeability

$\checkmark$ Permeability $x$ Thickness

$\checkmark$ Drainage area

$\checkmark$ Fracture half-length

All these variables can be visualized in a Relative Reservoir Quality Index Map - RRQI Map. (Refer to section 2.5.5 for a detail explanation of this Map). However, when an indicator is presented in the RRQI map is important to select the appropriate partitioning. 
Confirming that the variable under study has been partitioned properly requires a detailed evaluation of said indicator in each sub-zone (High, Mid-High, Mid-Low...etc). Therefore, the average value of the indicator evaluated needs to be revised in order to verify that is decreasing as the zone loses productivity.

An example is presented in Fig. 21. It shows an RRQI map of First year Cumulative. Once the partitioning has been selected it is necessary to verify that the field-wide pattern recognition is working properly under that specific delineation. To do so, the results of that partitioning and the average value of the indicator were checked in a zone-by-zone basis (right picture). As illustrated, the amount of hydrocarbon reduces progressively as the zone decreases its quality index.
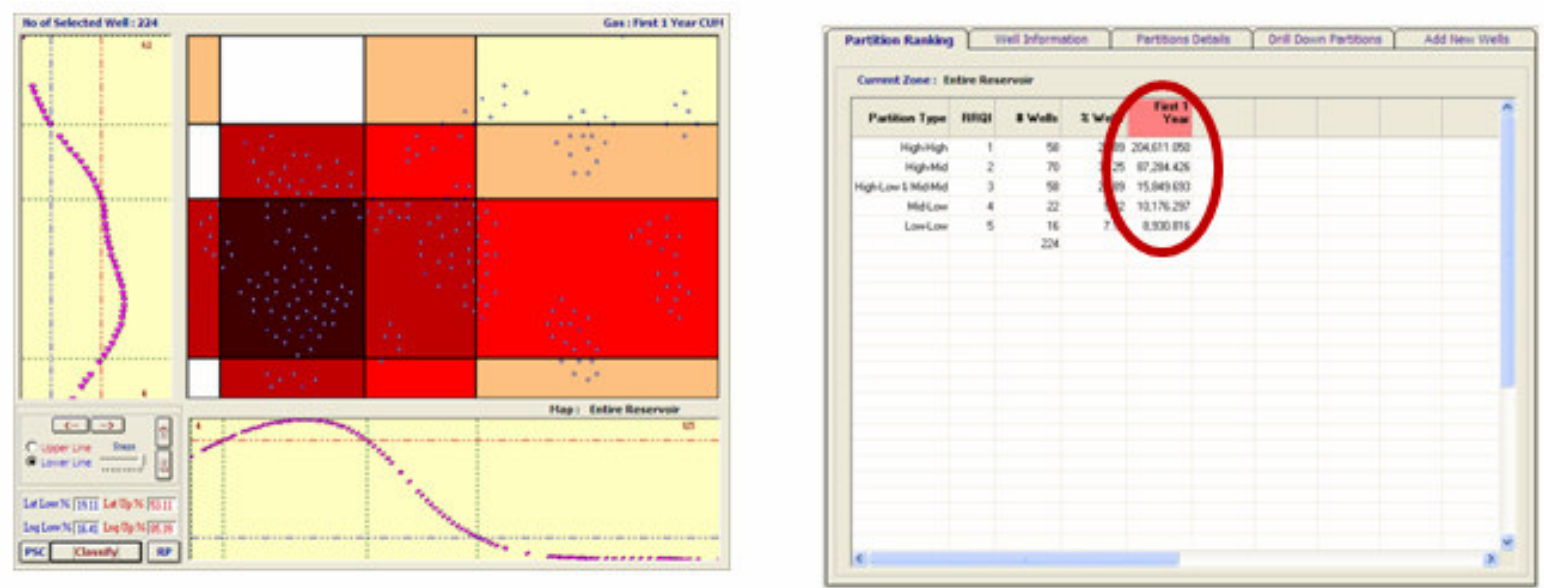

Fig. 21 - Revision of an appropriate partitioning.

Most of the results were compared employing the RRQI map. However, in some cases, the 3D-surface map provided a better visualization. In this case, the 3D-surface chart does not require a partitioning of the reservoir. Therefore, when these maps were used, the previously described process was not necessary.

At this point, the production data generated using the simulator was employed for performing an IPDA. Three conventional techniques had been 
used for analyzing the data and the field-wide pattern recognition interconnected the results. The analysis was completed.

The next step was comparing the IPDA results with the results from the real situation hosted in the simulator. 


\subsection{Evaluation of Results}

The IPDA technique provides a set of reservoir and well parameters, as well as tools for monitoring the well performance. It was necessary to include all these variables in the verification process for this validation study. Results encountered were divided into three categories.

\subsubsection{First set of results - Reservoir properties.}

The first set measured the capacity of the IPDA technique to:

$\checkmark$ Determine the initial Gas distribution.

$\checkmark$ Forecast remaining reserves at any given time.

$\checkmark$ Recognize intrinsic reservoir properties (permeability).

The distribution of the remaining reserves was compared after 30 years of production. The Estimated Ultimate Recovery will be compared after 45 years of production.

3.4.2 Second set of results - Well properties.

The second set targeted the predictions of well parameters, specifically:

\section{$\checkmark$ Fracture half-length \\ $\checkmark$ Drainage area.}

A special routine was designed for this section. In this case, the skin factor was used for altering the well performance. In a reservoir with 100 wells, half of them produced with a skin of +3 (in order to reduce their effective radius), whereas the other half produced with a skin of -3 (so that they would behave as stimulated wells). 
The expected result for this routine was to encounter a zone in the reservoir (the one that hosted the stimulated wells) with higher drainage area and fracture half-length.

\subsubsection{Third set of results. - Well performance.}

The IPDA technique provides tools for reservoir and well assessment, and it was imperative to include them in the validation process. The first feature is called "Under-performer wells", and it monitors the productivity of each well to verify its production rate. The other tool, "Evaluation of what-if scenarios", estimates the decline parameters (QI, Di and b) of any location in the reservoir, so potential spots for infill drilling can be selected and hypothetical production may be predicted. Specific routines were designed to verify the accuracy of these tools.

\section{Under-Performer wells}

An under-performer is a well that is not producing at full capacity (refer to section 2.5.7 for detailed explanation). To evaluate the capacity of the IPDA in recognizing this condition, it was necessary to select a group of wells and somehow reduce the productivity during the simulation process. As a result, the under-performance behavior could be reflected in the production data.

Ten wells were selected in SLM-100 and a skin factor of +4 was added to each of them. The positive skin alters the behavior of the well, reducing its productivity. Then it was necessary to verify if the IPDA recognized this condition.

Evaluation of what-if scenarios. 
One of the aims of this study is to prove that using monthly production rate data, the IPDA technique is capable of keeping track of the migration of sweet spots in a reservoir at any given time, thus recognizing zones that may still have reserves, and therefore the potential for infill drilling.

What-if scenarios are a tool that predicts the decline parameters (Qi, Di and b) for any location in the reservoir. Therefore, if a good location for infill drilling is identified, this tool can be used for predicting the behavior of a well in that specific point. Furthermore, a complete suite of economic analysis is provided, facilitating the calculations and assessments of different circumstances.

To evaluate the accuracy of this tool the model SLM-100 was modified. This version had 100 wells uniformly distributed along the reservoir (See Fig. 22). It is obvious that there are spaces that have enough separation and represent excellent candidates to host new wells (considering only spacing as the relevant criteria).

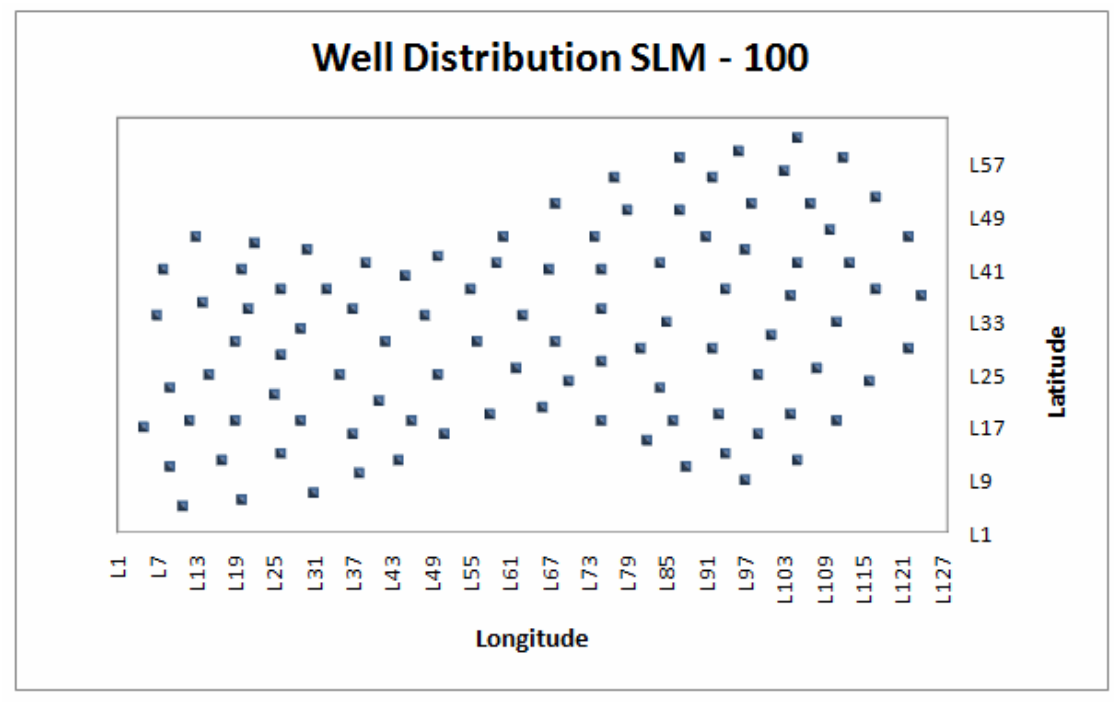

Fig. 22 -Well Distribution SLM 100.

Ten locations were selected in the reservoir, all located in zones with different characteristics (high permeability zones, low porosity zones, proximity to reservoir boundaries, etc...). 
Decline parameters were predicted employing what-if scenarios. In the controlled environment hosted at the simulator, ten wells were drilled located exactly in the same position.

In Fig. 23 the red dots indicate the locations selected. In this case, each place has different characteristics (some are close to the boundaries and others are in the center of the reservoir). The wells located close to the boundaries are placed in the Green Zone, and the red zone encompasses the wells separated from the boundaries.

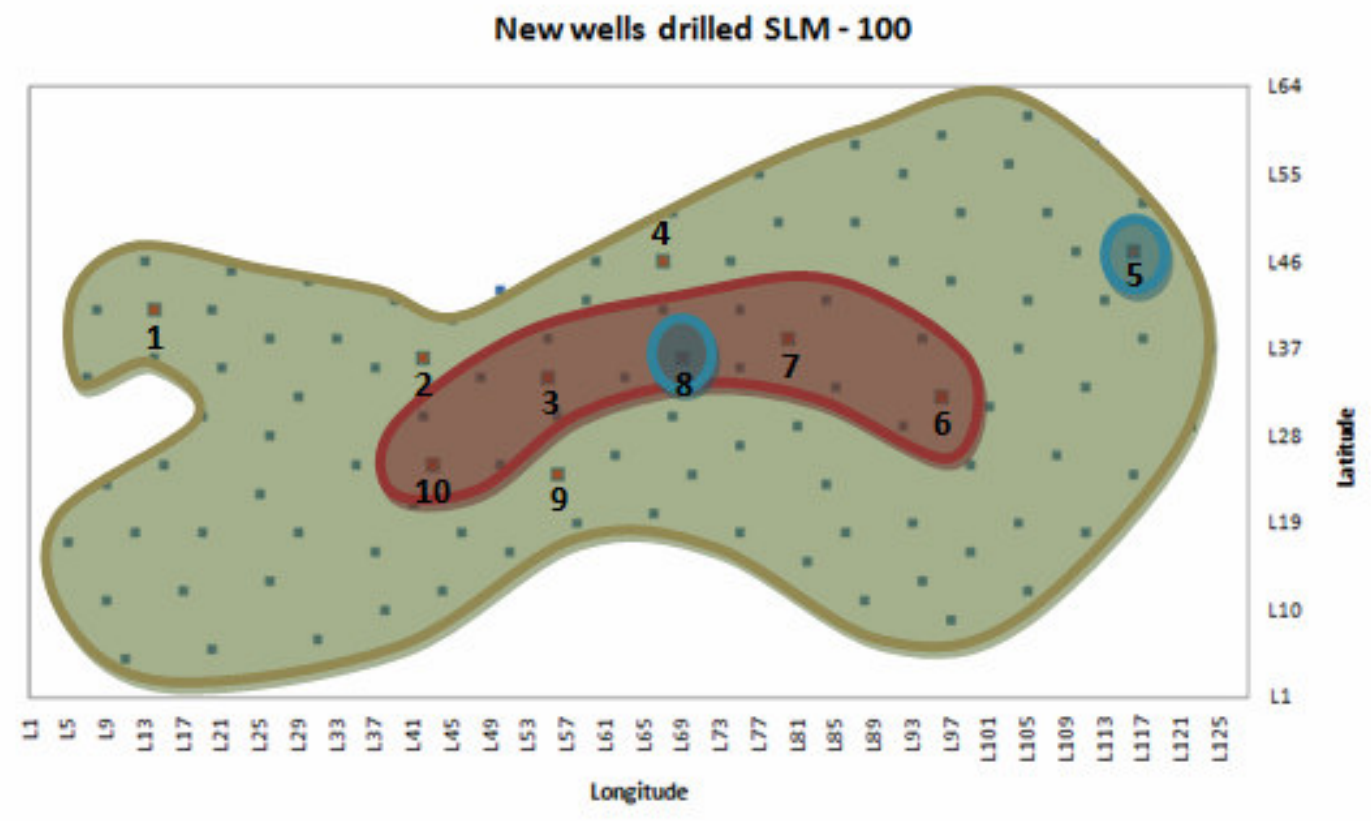

Fig. 23 - Location of the new wells.

The objective is to compare the production from the simulator and the modeled decline curve, measure the accuracy, and identify circumstances under which the tool provides incorrect recommendations.

It is also important to perform an economic analysis and measure quantitatively the accuracy of what-if scenarios. The Net Present Value will be used to conduct the economic assessments (refer to section 2.5.7 for detailed 
explanation). The wells encircled in blue represent two cases that will be evaluated in detail (See Fig. 23). 


\section{Chapter IV-Results and Discussion}

Using the IPDA technique, a set of formation parameters, well properties and performance indicators were calculated for each reservoir configuration included in this study. However, the purpose of this investigation was to determine if the values encountered corresponded to the actual situation in the reservoir. Hence, an effective comparison of results (IPDA vs. simulator) was imperative.

At this point, the IPDA analysis has been performed over five different reservoir configurations. For each model, six variables have been calculated. These parameters are listed below.

$\checkmark$ Initial gas distribution

$\checkmark$ Remaining gas distribution

$\checkmark$ Estimated ultimate recovery

$\checkmark$ Permeability

$\checkmark$ Drainage area

$\checkmark$ Fracture half-length

Also, a specific routine has been designed in order to determine if the IPDA technique is capable of detecting under-performer wells.

Finally, an additional model was created for the purpose of assessing the accuracy of the IPDA technique in predicting the behavior of future wells.

Up to this point, an enormous amount of information has been collected. Therefore, is necessary to organize the presentation of the results in accordance with the objectives stated for this investigation. Results will be shown in three groups: 
The first set of results will prove that the IPDA technique is capable of characterizing a reservoir and forecasting its future behavior. The variables assigned to this section are: initial gas distribution, remaining gas distribution, estimated ultimate recovery and permeability.

The second set of results will confirm the ability of this method for estimating individual well parameters. The variables evaluated in this section were: well drainage area and fracture half-length.

Finally, the third set of results will demonstrate that the IPDA successfully recognizes under-performer wells and that it can predict the behavior of future wells at any location. 


\subsection{First set of results - Reservoir properties.}

The first set of result includes four variables that monitor reservoir performance and formation properties. Those parameters are:

$$
\begin{aligned}
& \text { I - Initial gas distribution } \\
& \text { II - Remaining gas distribution } \\
& \text { III - Estimated ultimate recovery } \\
& \text { IV - Permeability }
\end{aligned}
$$

Results provided by the IPDA analysis will be compared to the actual values in the simulator to assess the ability of this technique to recognize reservoir properties in different situations.

\subsubsection{First set of results for Single Layer Model}

I - Initial gas distribution (IGD).

First, it was necessary to find the actual initial gas distribution. This information was read from the simulator. It was found, that at the time when the reservoir was discovered, the medium-left zone had the highest concentrate of hydrocarbon (See Fig. 24).

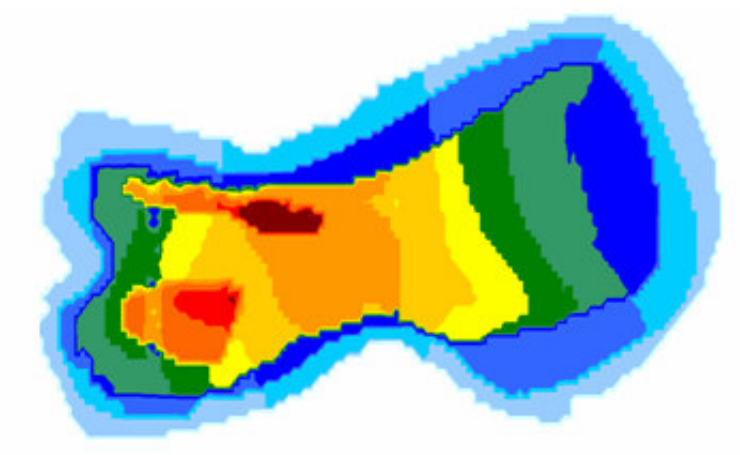

Gas in Place (MMsCF)

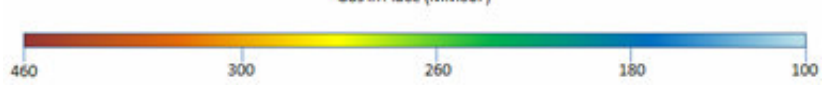


Fig. 24 - SLM. Actual initial gas distribution.

The SLM was produced using two different well configurations. One version had 224 wells (cluster-distributed) and the other 100 wells (uniformly distributed). The purpose of having two different well distributions was to determine the impact that this variable causes on the IPDA predictions.

The IPDA result for initial gas distribution in model SLM 224 is shown in Fig. 25. On the left you will find the Relative Reservoir Quality Index or RRQI. To facilitate the interpretation of the results, at the right you will find a map indicating the actual value (simulator) of this parameter. The same delineation was used for partitioning both maps, so the zones can be contrasted. In this case, the IPDA successfully indicated zone 1 as the region with highest gas concentration.

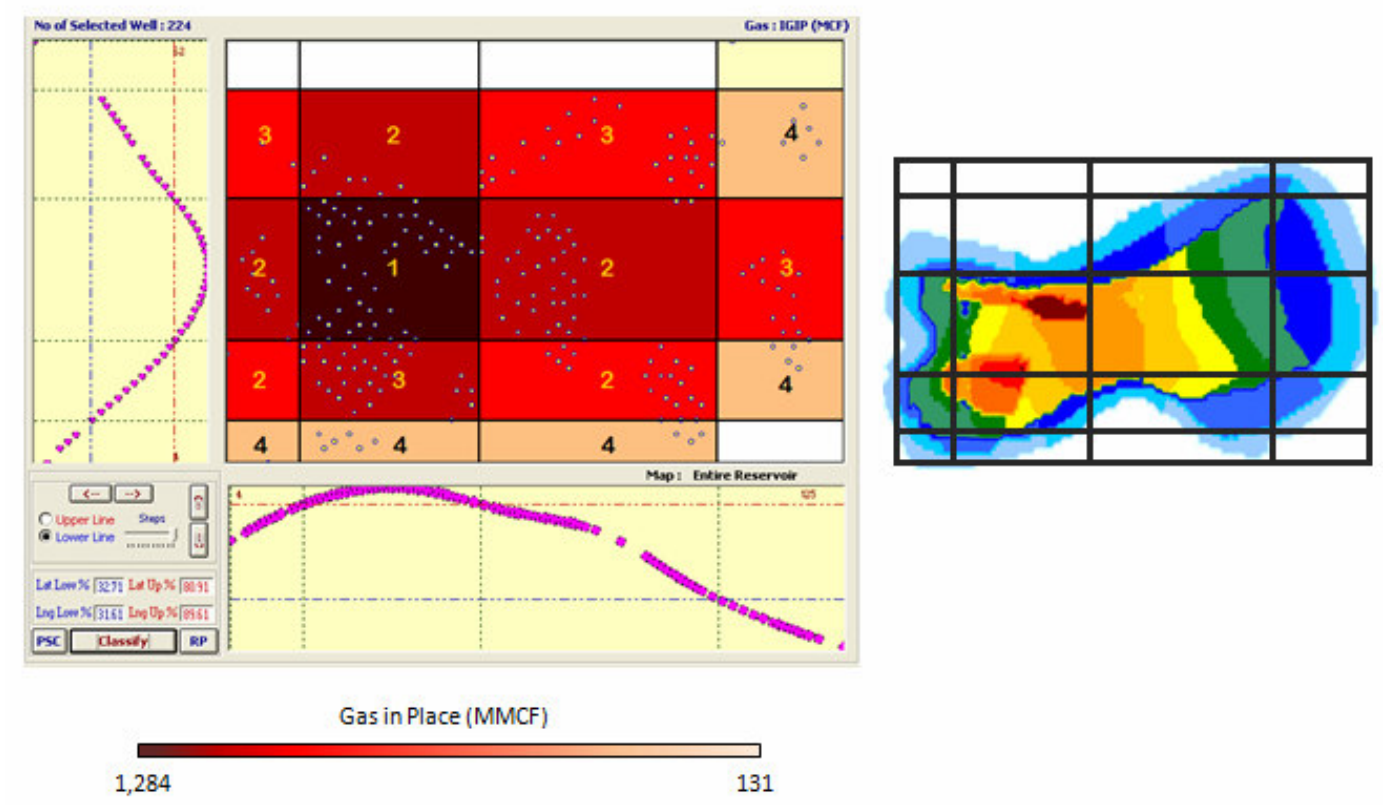

Fig. 25. RRQI SLM-224. Initial gas distribution.

A similar behavior was expected in the second version of the single layer model (SLM-100). 
Fig. 26 represents the RRQI map of the IPDA result for initial gas distribution, this time for model SLM-100. Again, the IPDA was able to identify the sweet spot, recommending the mid-left region as the premium zone.
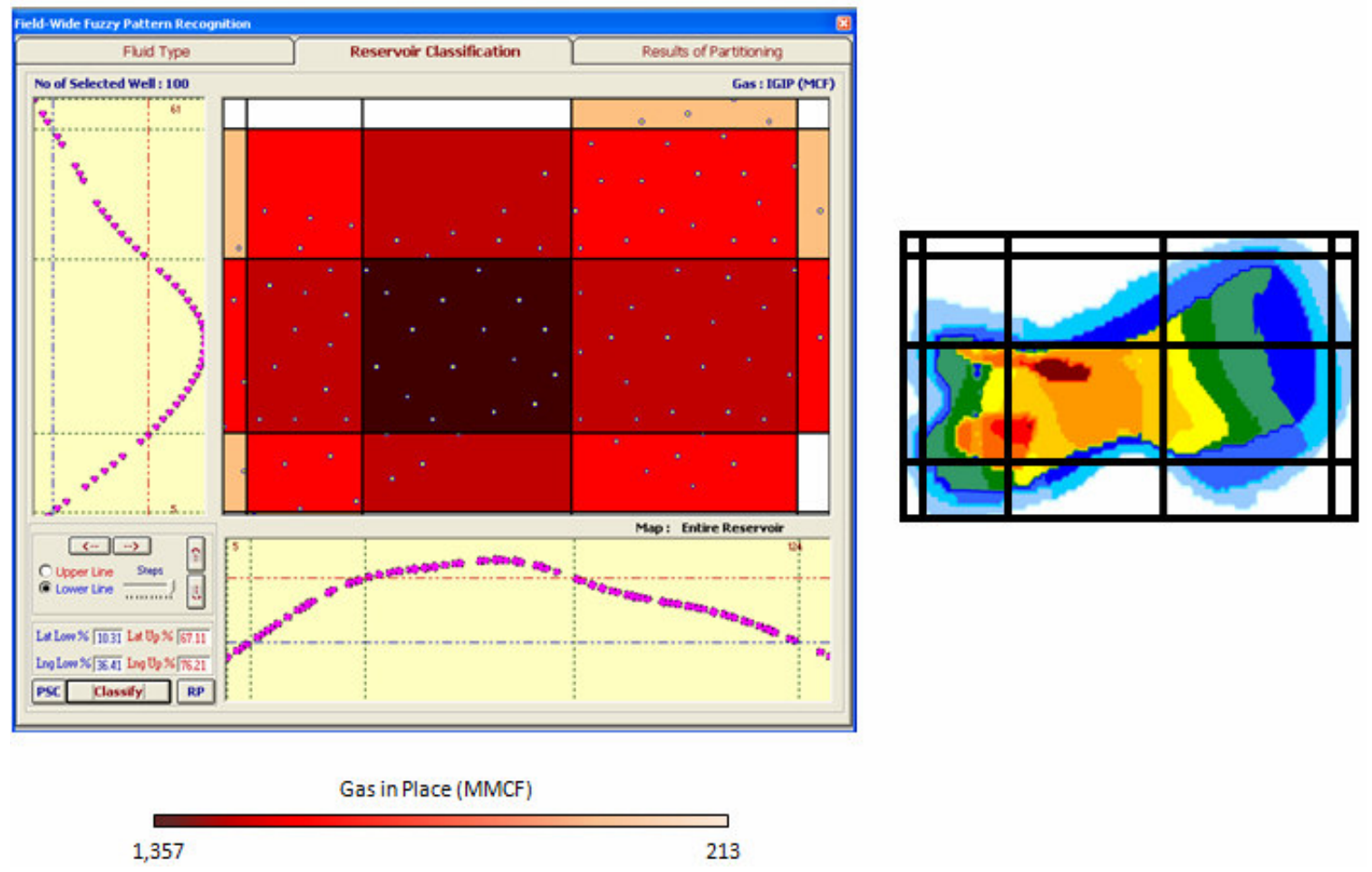

Fig. 26 - RRQI SLM-100. initial gas distribution

These results confirmed one of the initial hypotheses of this research, the fact that the well distribution does not determine the accuracy of the IPDA predictions.

\section{II - Remaining Reserves.}

Once the initial gas distribution was successfully identified, it was time to determine if the IPDA technique was capable of tracking the migration of the sweet spots in the reservoir. To do so, the remaining gas distribution after 30 years of production was assessed.

The new gas distribution after 30 year of production was read from the simulator, the results are shown in Fig. 27. 


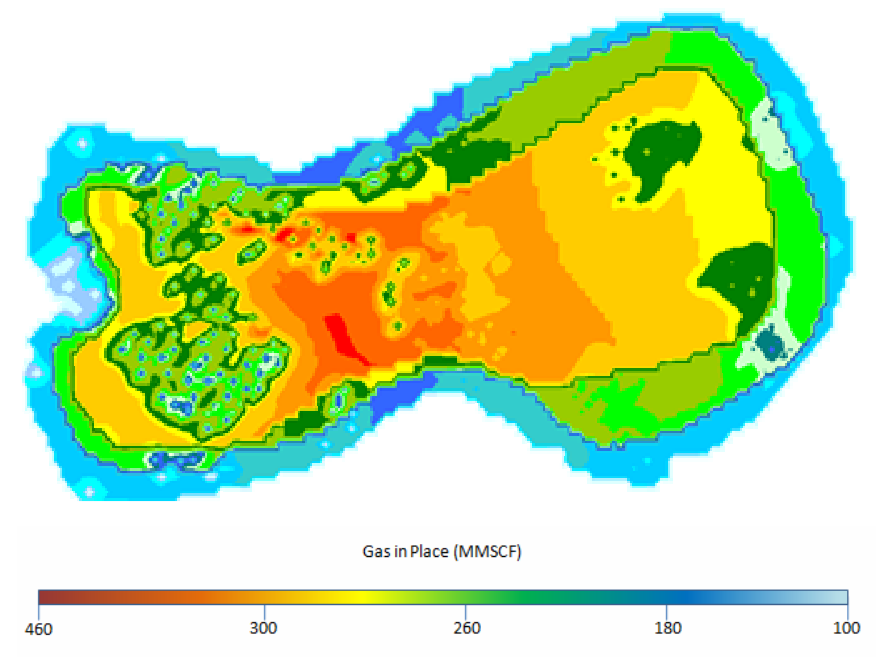

Fig. 27 - SLM. Actual Remaining Gas Distribution.

When the initial gas distribution was evaluated, it was found that the zone with the highest amount of reserves (premium zone) was located at the left region of the reservoir (See Fig 24). However, after 30 years of production, the premium zone had migrated to the center region of the field, because the left part had been drained quicker. This was a good opportunity to determine if the IPDA technique was able to detect this issue.

Using the IPDA technique, the remaining gas distribution after 30 years of production was estimated. For both versions, SLM-224 and SLM-100, the RRQI maps were obtained.

Fig. 28 illustrates the RRQI of $S L M-224$ for remaining gas distribution after 30 years of production. As is illustrated, the premium zone (dark brown) has moved to the center region of the reservoir, which indicates that the IPDA successfully predicted the migration of sweet spots in the field. At the right, you 
will find the actual gas distribution read from the simulator. Again, the same partitioning was used for both maps ( $R R Q I$ and actual gas distribution) so subzones can be easily recognized.

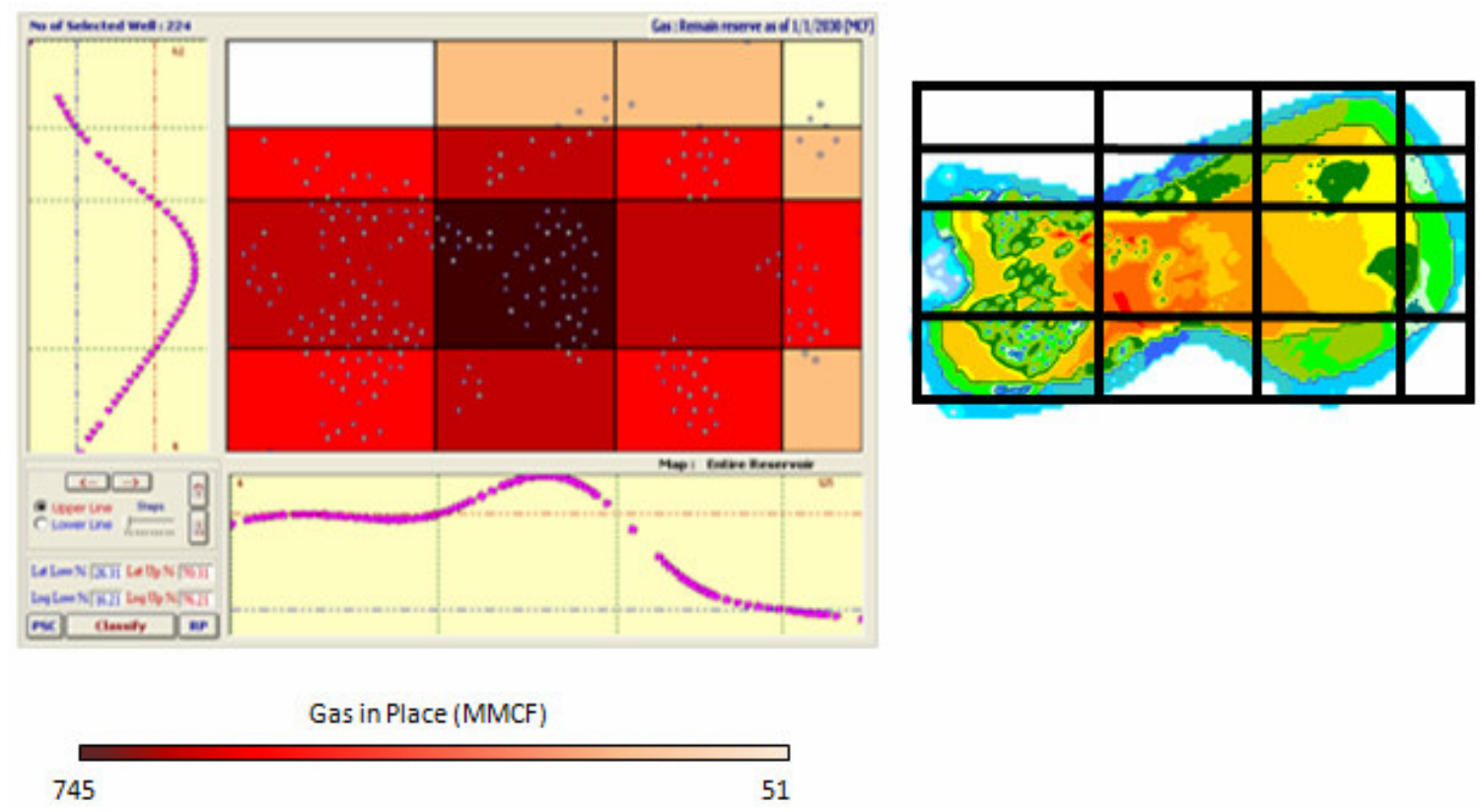

Fig. 28. RRQI SLM-224. Remaining gas distribution after 30 years of production.

For the second version of the single layer model, SLM-100, the same results were expected. The results are presented in Fig. 29 and, again, the migration of the remaining gas distribution was also recognized. In this version, the RRQI (left) and the actual map once again coincide in identifying the premium zone in the mid-region of the reservoir.

Three conclusions can be stated after these findings. First, that the IPDA is capable of indicating the premium zones (highest concentration of hydrocarbon). Second, that this technique can keep track of the fluid flow at any time. And finally, it has been demonstrated that the well distribution does not affect the ability of the IPDA to recognize sweet spots in a field. 
The noted conclusions partially validate one of the aims stated at the beginning of this project. Now, is necessary to determine if the same results are achieved for the Two-Layer Mode.

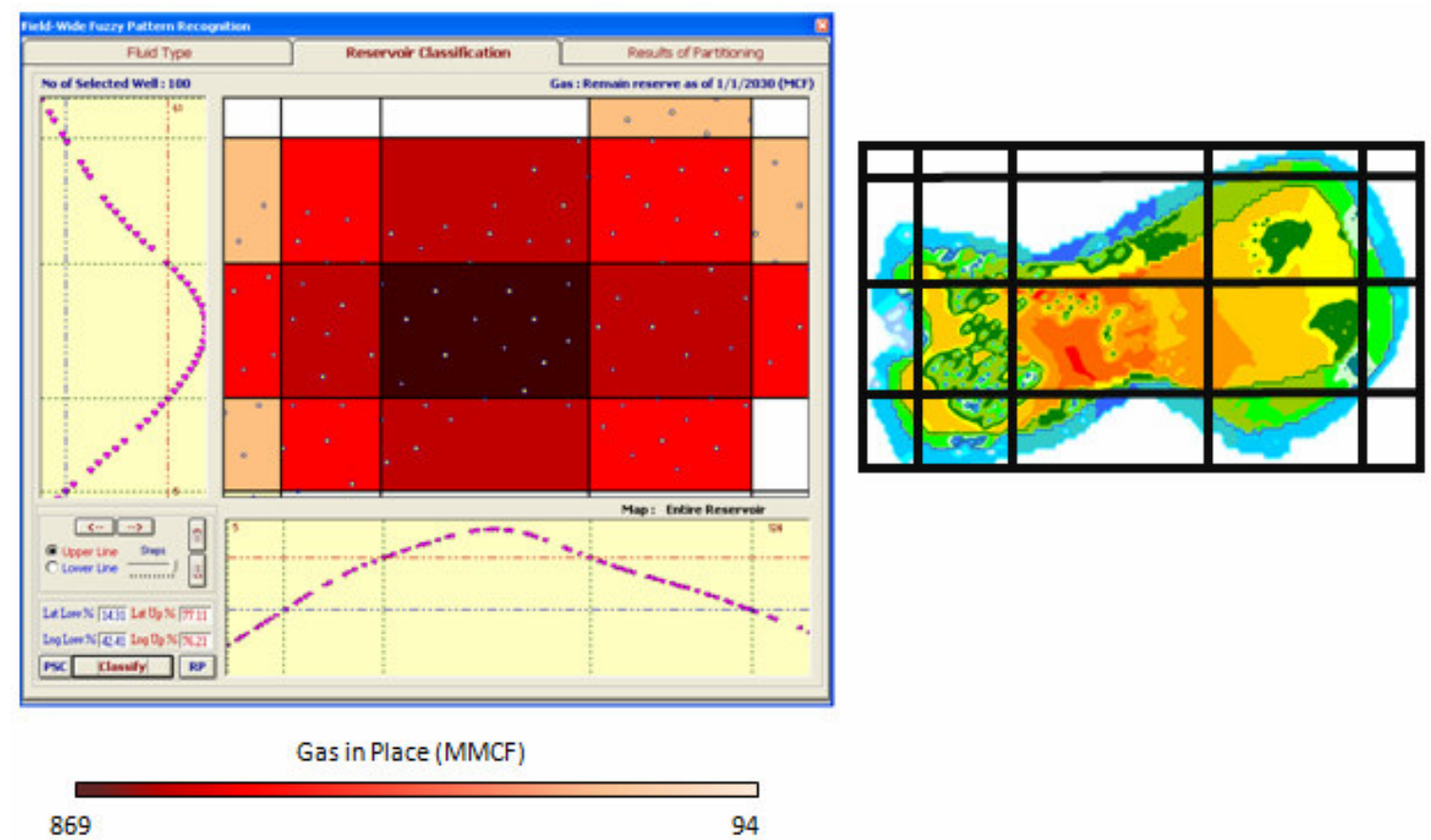

Fig. 29. RRQI SLM-100. Remaining gas distribution after 30 years of production.

III - Estimated ultimate recovery.

The estimated ultimate recovery EUR is the total amount of gas that has the potential to be produced up to the termination of the life-time in a well. The IPDA performs this calculation based on the decline curve analysis.

To validate the ability of the IPDA to successfully estimate this indicator, the EUR of the wells was compared after 45 years of production. The technique employed compared the EUR at 45 years with the cumulative production (after the same period of time) recorded in the simulator. This process was performed in a well-to-well basis. 
To quantify the error of this prediction, the percentage of difference between EUR (estimated using the IPDA) and the actual cumulative production (reported by the simulator) was calculated.

The first case studied was the SLM-224. In this model, the EUR predicted using the IPDA technique was found to be higher than the cumulative production. However, the average difference was measured to be $7.39 \%$.

The results for SLM-224 are presented in Fig. 30. The orange zone represents the 45 years of cumulative production recorded in the simulator. On the other hand, the blue zone is the 45 years EUR calculated with the IPDA technique.

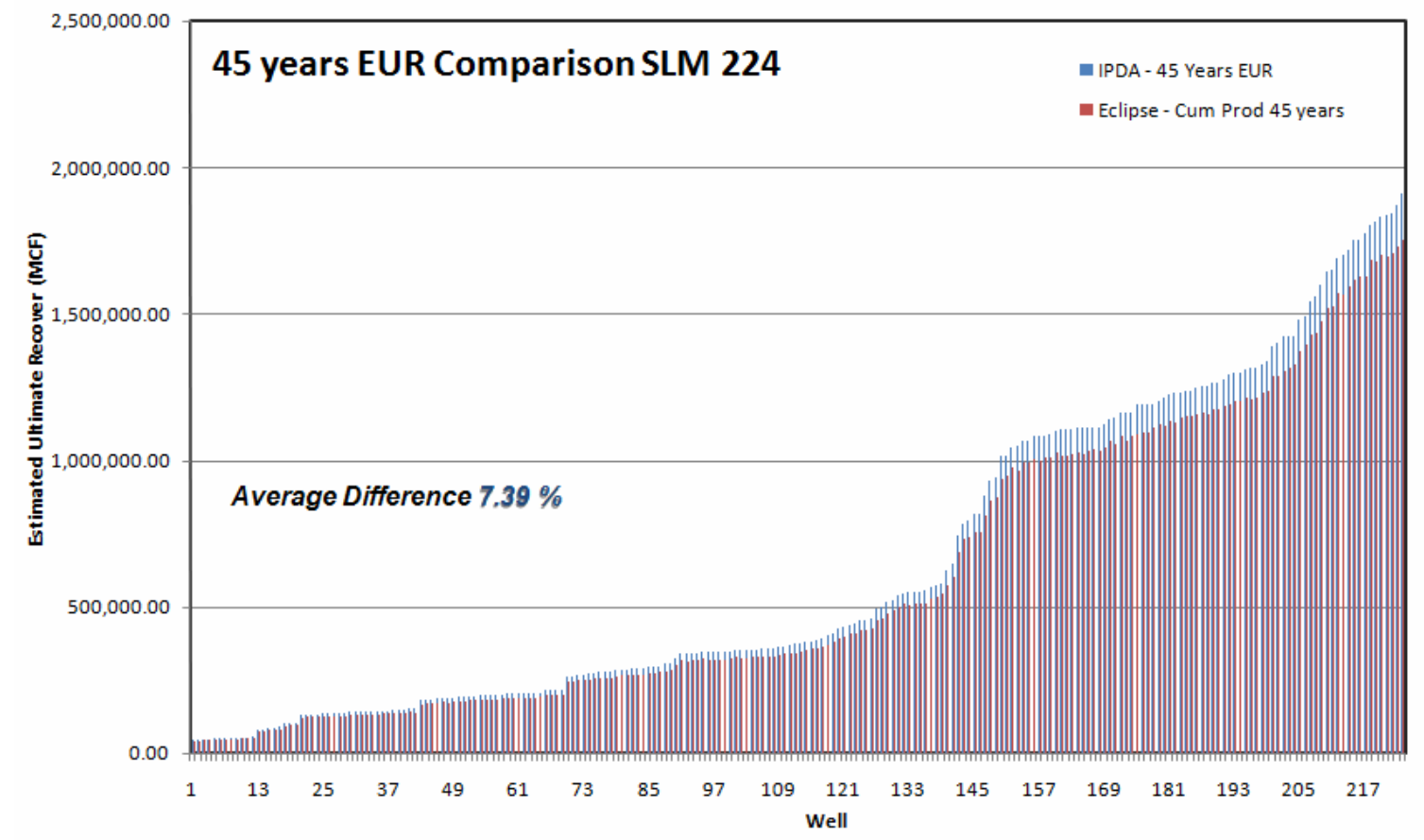

Fig. 30 - SLM-224. Evaluation of 45 years EUR.

The same procedure was repeated for model $S L M-100$. It should be mentioned here that the IPDA tended to overestimate the EUR value (the same situation encountered in version SLM-224). However, in this model the average 
difference between the IPDA predictions and the actual value registered in the simulator was found to be $6.44 \%$. Fig. 31 shows these results.

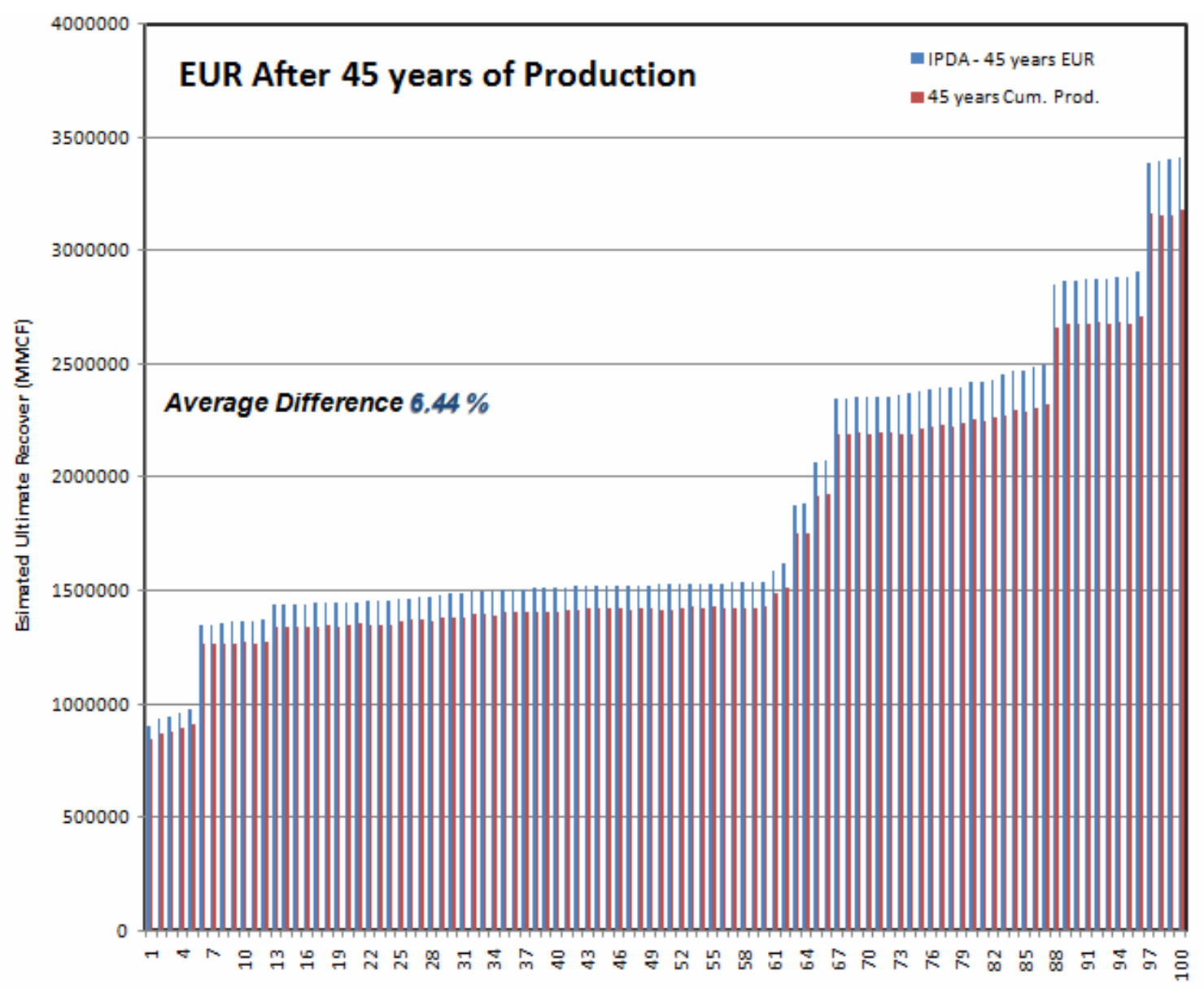

Fig. 31 - SLM-100. Evaluation of 45 years EUR.

After completing the EUR verification, the conclusion is that the IPDA technique is capable of forecasting EUR with a minimum percentage of error (less than 10\%). The difference is acceptable, considering that these estimations were calculated based only on records of monthly production rate data.

The second issue that should be mentioned is the tendency of the IPDA technique to overestimate the EUR value. The origin of this problem is most likely the hyperbolic index $b$. The majority of the decline curves performed on this model registered a $b$ value greater than 0.5 . As a result, when the decline is 
extrapolated, this high $b$ affects the behavior of the curves "tail", which causes the overestimation.

\section{IV-Permeability.}

The permeability $(k)$ distribution of the single layer model was quite uniform, showing three spots of high $k$ (See Fig. 32). The objective in this case was to prove that the IPDA technique was able to recognize these three zones.

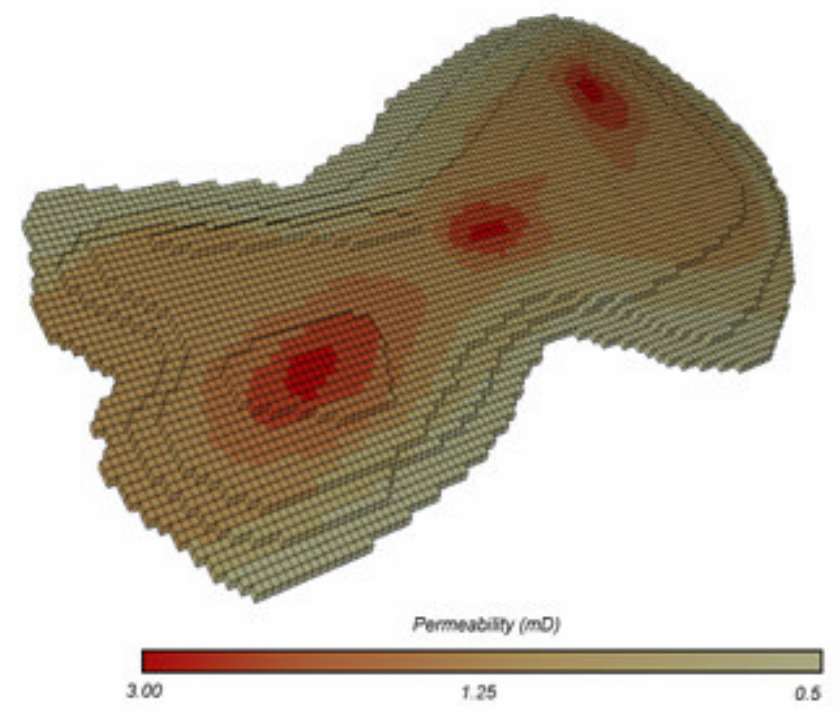

Fig. 32 - SLM. Permeability Distribution.

The permeability distribution of the single layer model was estimated based on the information provided during the process of type curve matching. However, when the permeability indicator was evaluated in the RRQI map, it was not possible to come up with a partition that highlighted the three zones with high $k$. The same situation occurred in both SLM-224 and SLM-100.

Fig. 33a illustrates the RRQI of the permeability for SLM-224. The picture at the right represents the actual permeability of the single layer model. Notice that the partitioning does not allow visualizing the three zones with high $k$ values. This situation was similar in both versions of the single layer model. 

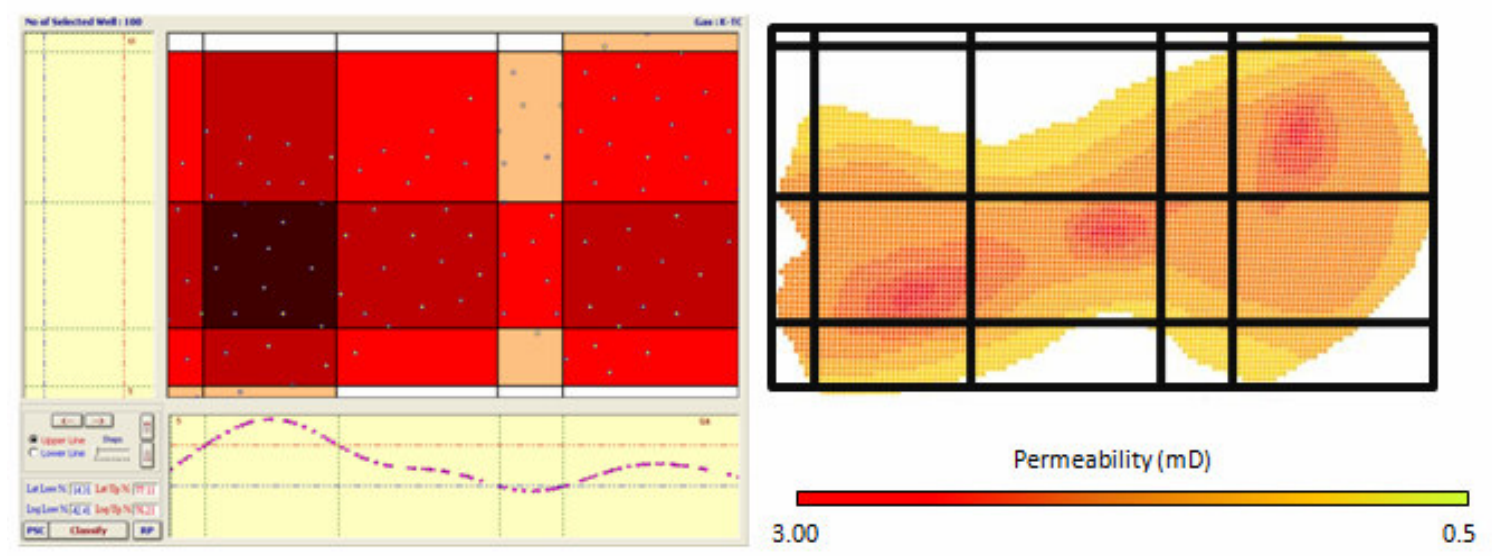

Permeability (mD)

1.5

Fig. 33a - RRQI SLM 224. Permeability Distribution.

However, the fact that the permeability distribution could not be partitioned properly in the RRQI map did not mean that these calculations were necessarily incorrect. The problem could have been related to the configuration of the RRQI map.

A commercial type of software for advanced graphing was used to determine whether the $k$ estimations were correct or not. The program employed is called Xyfunction, and it uses advanced triangulation for connecting the data points.

When the permeability values were plotted using XYFunction, the results showed a notable similitude with the actual $k$ values. This proved that the permeability estimations were correct and the problem was related with the configuration of the RRQI map.

The results of permeability estimation were plotted using the Xyfunction, and are shown in Fig. 33b. Notice that the three spots of high $k$ are identified. 


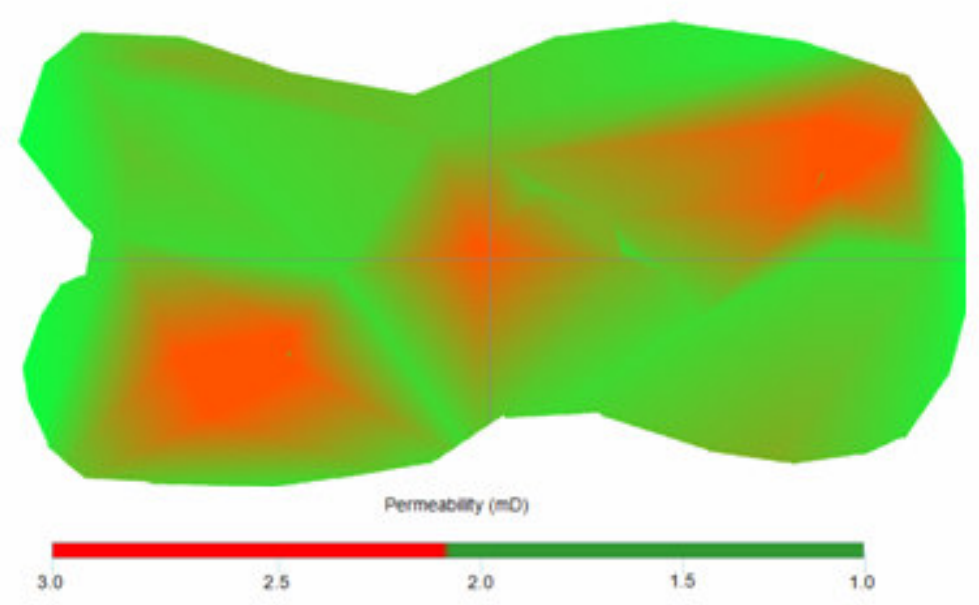

Fig. 33b -SLM-224. Permeability Distribution.

The permeability values calculated through the IPDA technique did not coincide numerically with the actual $k$ values. In fact, the permeability calculated by the IPDA was always found to be lower. This required a detailed evaluation of this indicator in a well-to-well basis.

It was found that that the permeability obtained through the IPDA technique reflected a fall when compared to the actual $k$ values. However, this difference was consistently present in all the calculations.

Both indicators were plotted in Fig. 34. It was found that the predicted $k$ and the actual $k$ followed a similar trend. The blue line represents the actual permeability (simulator), and the orange line represents the values estimated by the IPDA. The correspondence observed in Fig. 34 demonstrates that the IPDA technique is capable of deducing the permeability distribution present in the reservoir. 
Permeability Correspondence

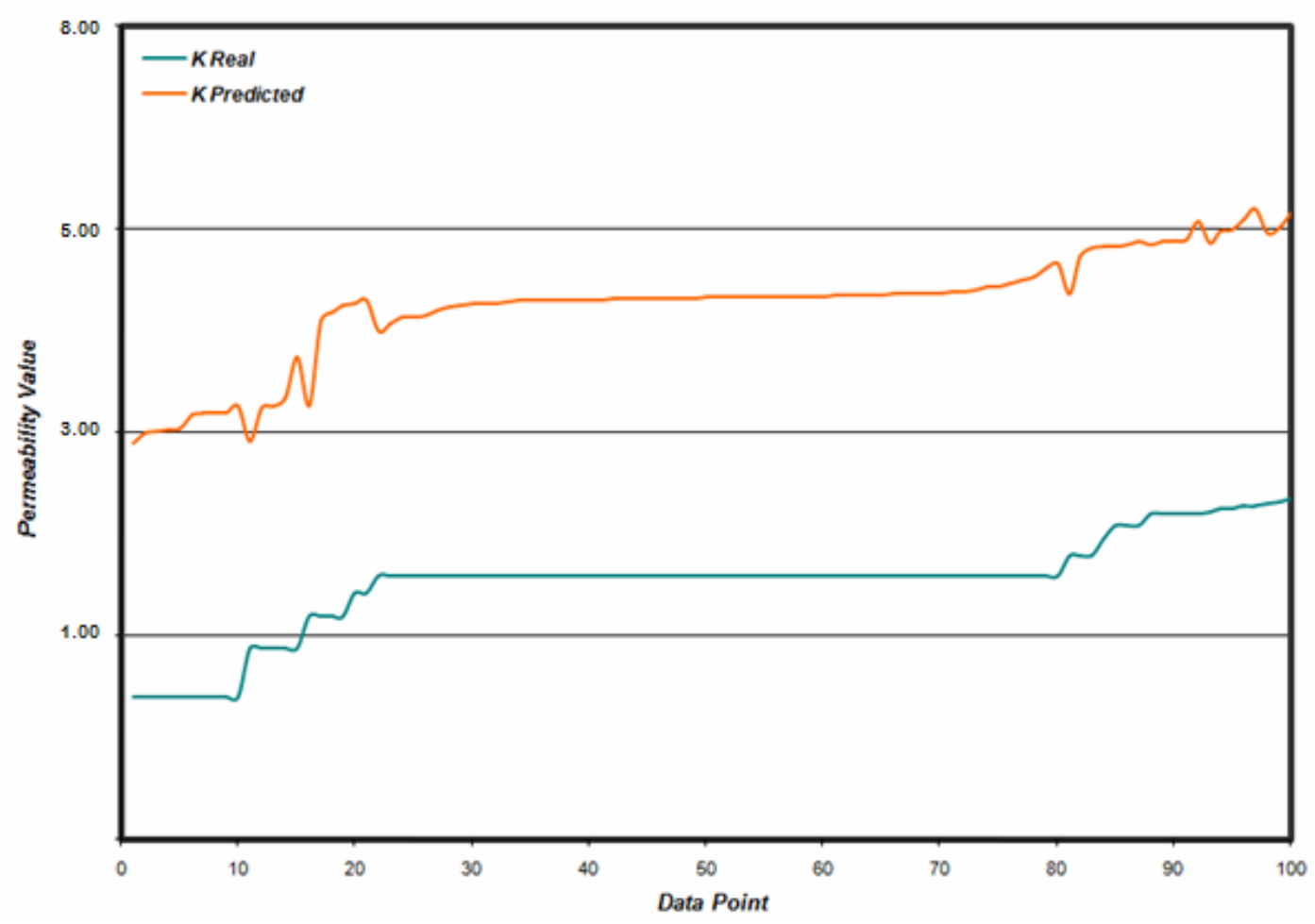

Fig. 34 - SLM-100. Permeability trend.

The first set of results was completed. In this section, it has been demonstrated that for single layer formations the IPDA technique is capable of:

$\checkmark$ Characterizing a reservoir

$\checkmark$ Forecasting future behavior

\subsubsection{First set of results for Two-Layer Model.}

The Two-Layer Model had three versions. The objective was to evaluate the ability of the IPDA technique to recognize intrinsic properties in a reservoir. Therefore, in each version a property of the formation (either permeability or porosity) was set to be homogeneous. 
TLM-1 had homogeneous porosity and heterogeneous permeability. TLM2 had homogeneous permeability and heterogeneous porosity. Finally, TLM-3 had both porosity and permeability with heterogeneous distribution.

\section{I - Initial gas Distribution.}

The version TLM-1 had homogeneous porosity. Therefore, the initial gas distribution was uniform. The IGD was read from the simulator and is shown in Fig. 35.

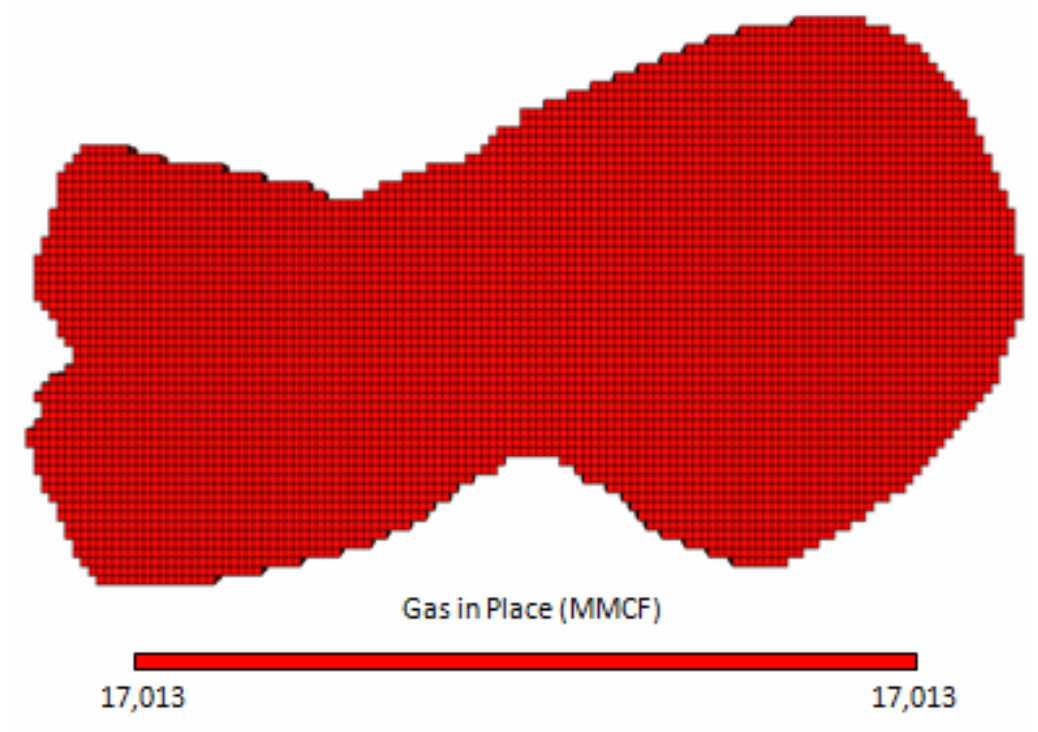

Fig. 35 - TLM-1. Actual Initial Gas Distribution.

The purpose of this version was to evaluate the performance of the IPDA technique in homogenous formations. The estimation of the IPDA for initial gas distribution in TLM-1 is shown in Fig. 36.

This time, the results are presented on a 3D-surface graph. The map shows a uniform distribution of gas in the reservoir. The right zone presents a small depletion, but this difference is not significant. IPDA is not an exact technique, so these small variations are normal and they do not compromise the integrity of the analysis. 


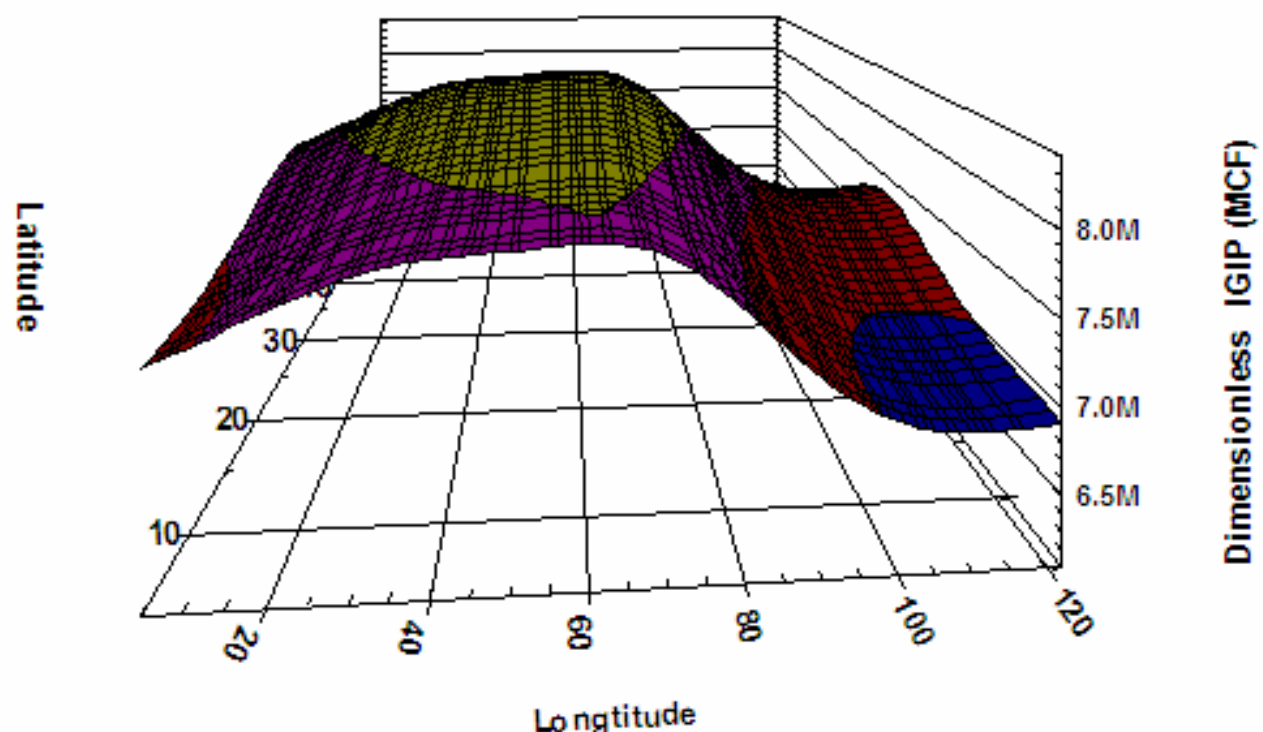

Gas in Place (MMCF)

Fig. 36 - 3D-surface-TLM-1. Initial Gas Distribution.

The other versions of the Two-Layer Model (TLM-2 and TLM-3), however, were designed with heterogeneous porosity. In these models the Initial gas distribution was not constant; the left zone had a higher concentration of gas. Fig. 37 illustrates the actual IGD for these two versions.

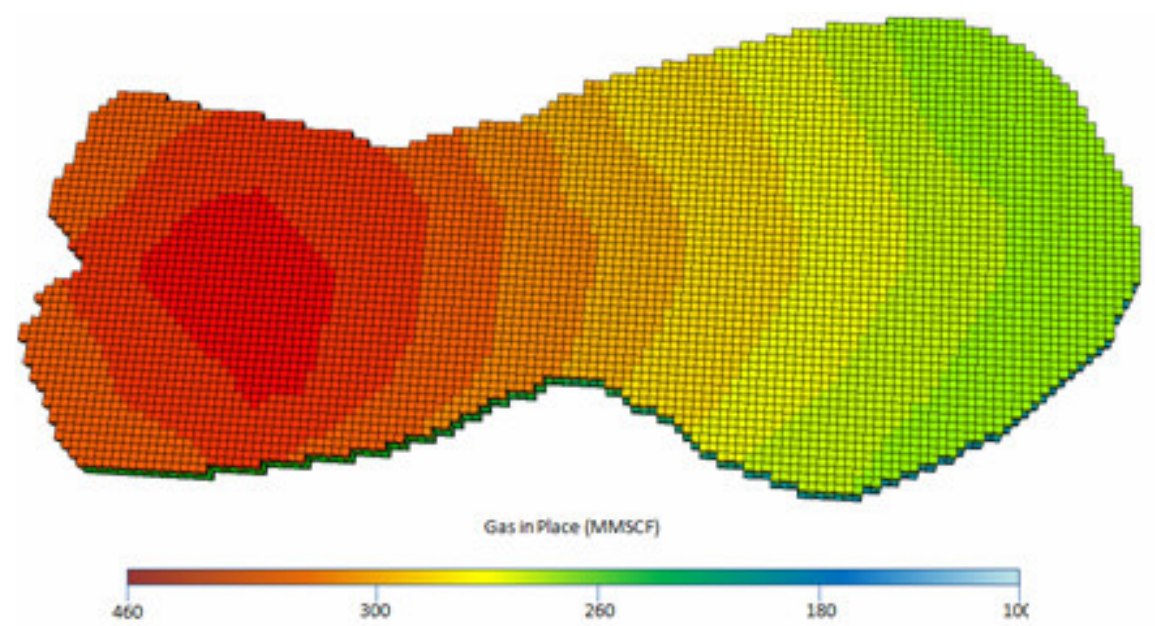

Fig. 37 - TLM-1 \& TLM-2. Actual Initial Gas Distribution. 
The IPDA successfully recognized the gas distribution in this model. Fig. 38 shows the RRQI map of Initial gas distribution for TLM-2. At the right there is a map indicating the actual IGD (from simulator). The RRQI map identified the premium zone.

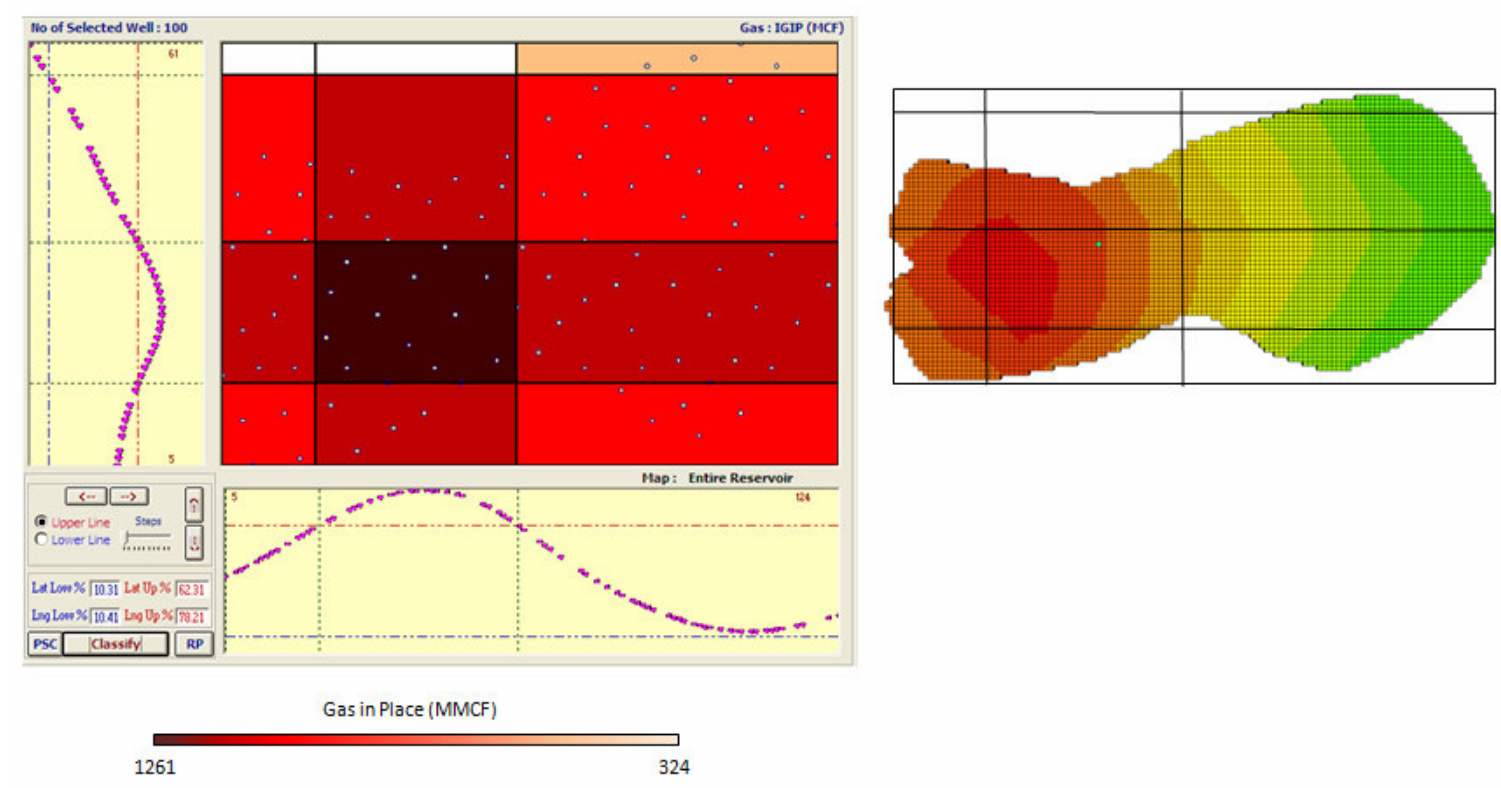

Fig. 38 - RRQI TLM-2. Initial Gas Distribution.

II - Remaining gas distribution.

At this point, it has been proven that the IPDA recognizes initial gas distribution, regardless of the characteristic of the formation.

For TLM-1, it was found that the IGD had a uniform distribution. This model was produced for 26 years and the distribution of the remaining reserves changed drastically.

The first step was to evaluate the actual distribution of gas reserves in the simulator. After 26 years of production, the premium zone in model TLM-1 had moved to the middle region of the reservoir. This migration is illustrated in Fig. 39. Two zones have been drained faster (indicated by the color blue), because in these regions the permeability is higher. 


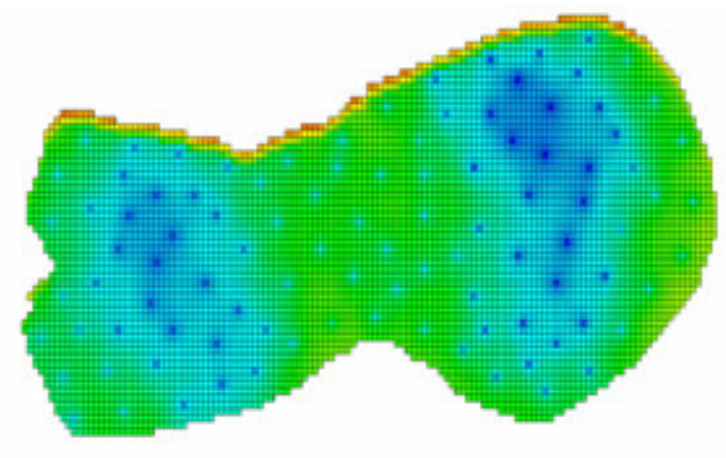

Gas in Place (MMCF)

Fig. 39 - TLM-1. Gas Distribution after 26 years.

Fig. 40 illustrates the RRQI of $T L M-1$ for remaining gas distribution after 26 years of production. As is obvious, the premium zone (dark brown) has moved to the center region of the reservoir, which indicates that the IPDA recognized the new location of the sweet spots in the field. At the right, you can find the actual gas distribution read from the simulator.

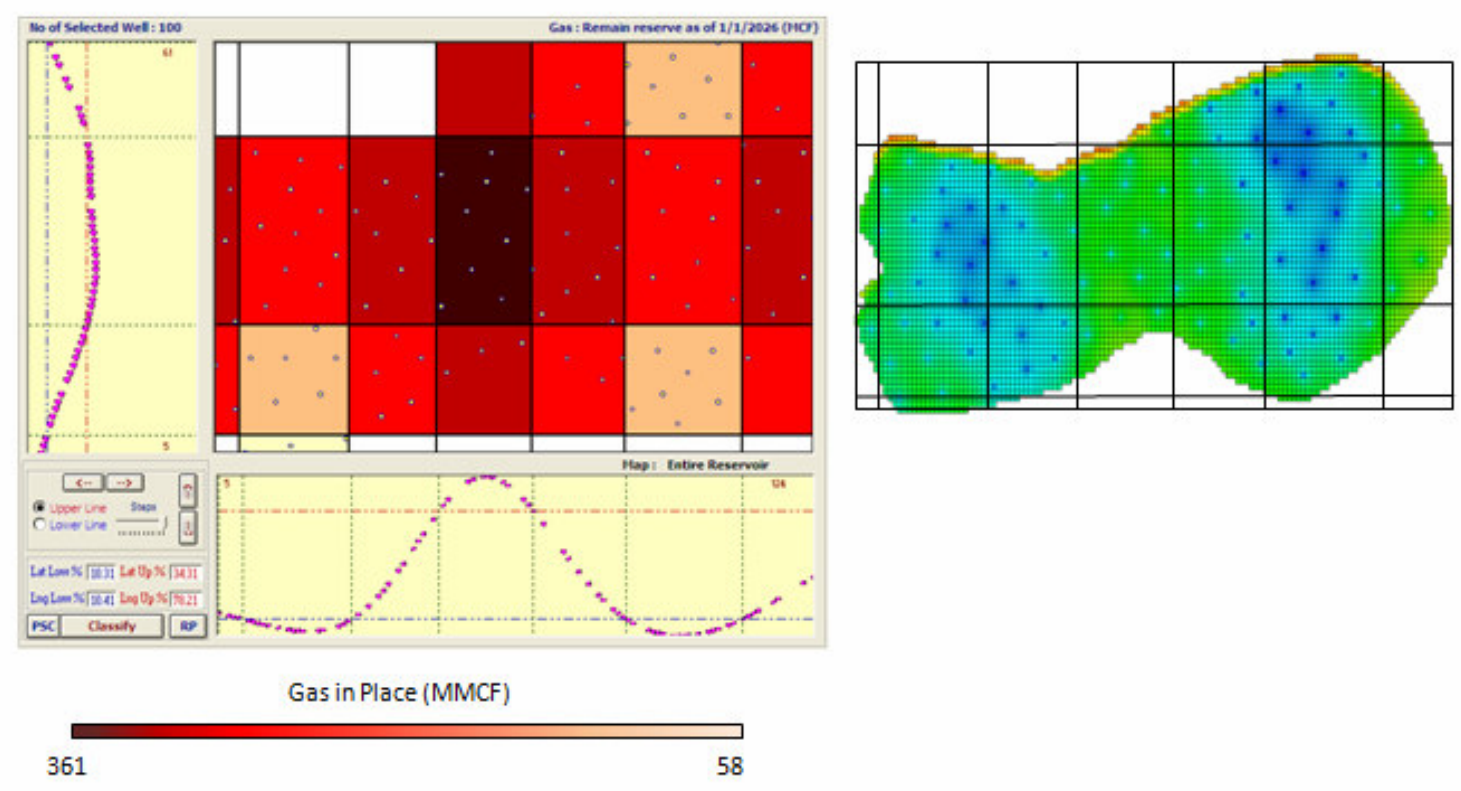

Fig. 40 - RRQI TLM-1. Gas Distribution after 26 years. 
The same procedure was repeated for $T L M-2$ and $T L M-3$. In these models, the initial gas distribution indicated that the left-region was the premium zone (See Fig. 37).

Since the permeability for model $T L M-2$ was homogeneous, the gas distribution should remain identical. This situation was corroborated in the simulator.

The results estimated by the IPDA recognized this issue. The 3D-surface map in Fig. 41 (left) corresponds to the remaining gas distribution after 26 years for TLM-2. This map shows a higher amount of reserves in the left-zone of the reservoir and a pronounced depletion at the right area.
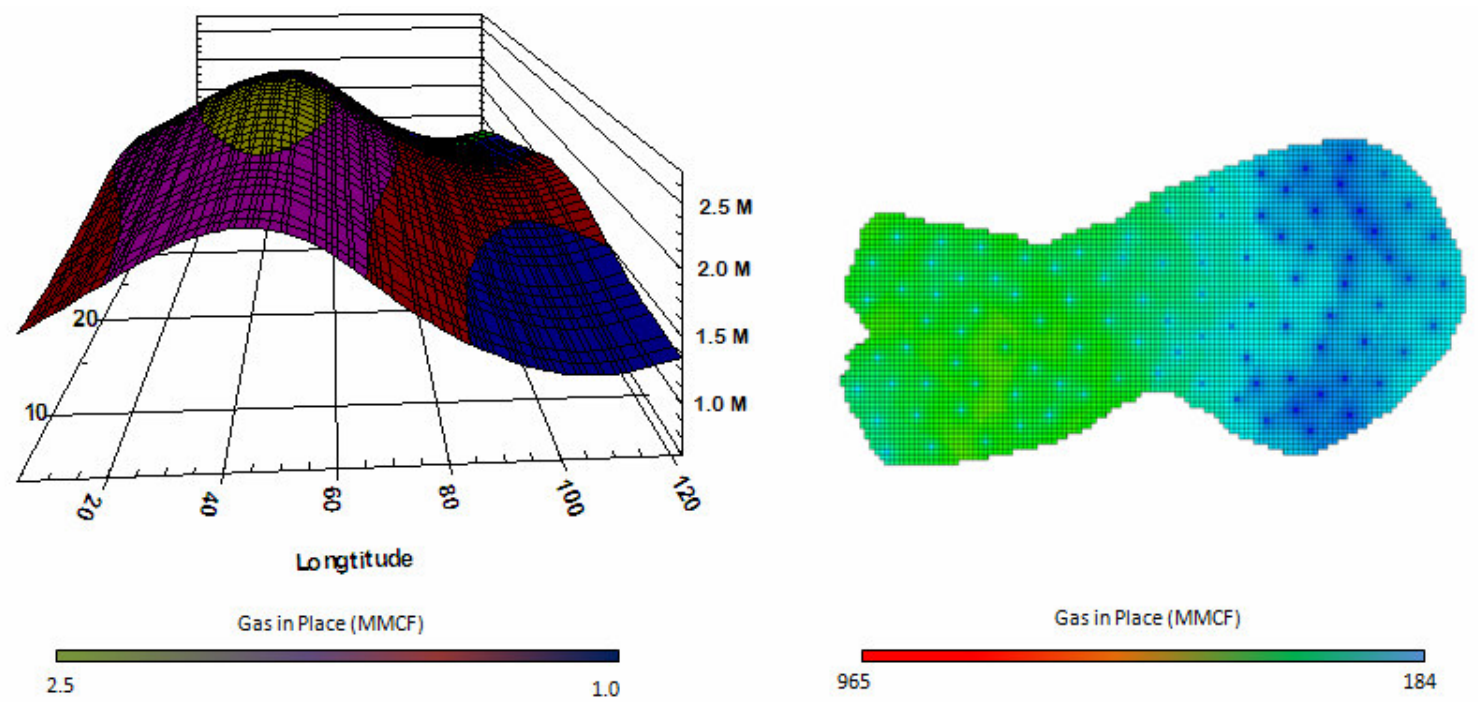

Fig. 41 - TLM-2. Gas Distribution after 26 years.

In addition, the graph in Fig. 41 (right) represents the actual remaining gas distribution found in the simulator. Notice that both graphs indicate that the leftregion is the premium zone in the reservoir. 
Up to this point, the gas distribution in two multi-layered formations has been determined (at different times) using Intelligent Production Data Analysis. The correspondence of these results with the actual situation in the simulator has also been demonstrated.

Finally, the third version of the Two-layer Model, TLM-3, was evaluated. The initial gas distribution is similar to the one found in TLM-2 (See. Fig. 37). In the previous versions of Two-Layer Model (TLM-1 and TLM-2), a reservoir property (either porosity or permeability) was homogeneous, so the gas motion could be seen as function of time.

On the other hand, version TLM-3 had a heterogeneous permeability, with a different distribution in each layer. This time, there was no way to predict the behavior of the reserves. The actual remaining gas distribution was read from the simulator and is shown in Fig. 42.

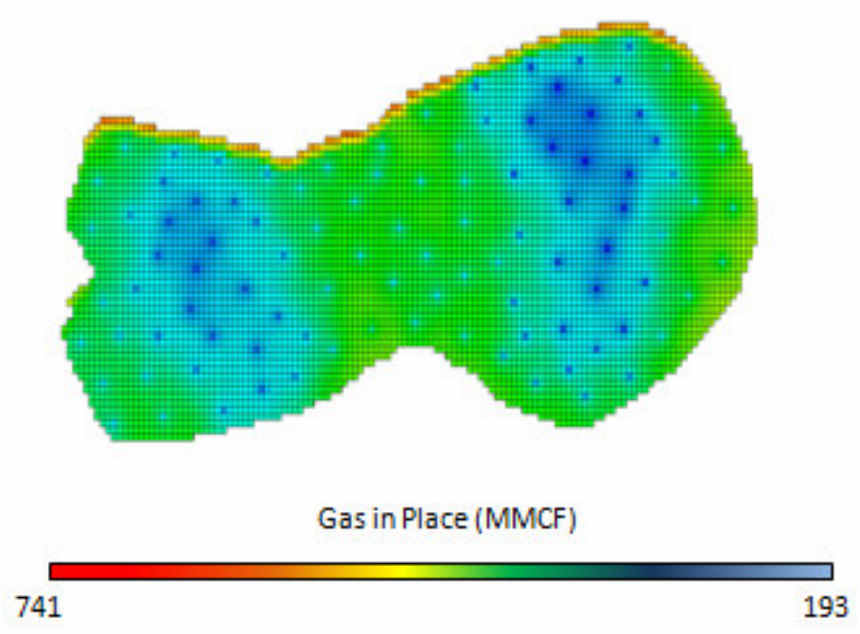

Fig. 42 - TLM-3. Actual Gas Distribution after 26 years.

Then, using the IPDA, the remaining gas distribution was calculated. The results are shown in Fig. 44. The RRQI map has a remarkable coincidence with the actual situation found in the simulator. Not only the premium zone, but also the depleted regions were successfully identified. 


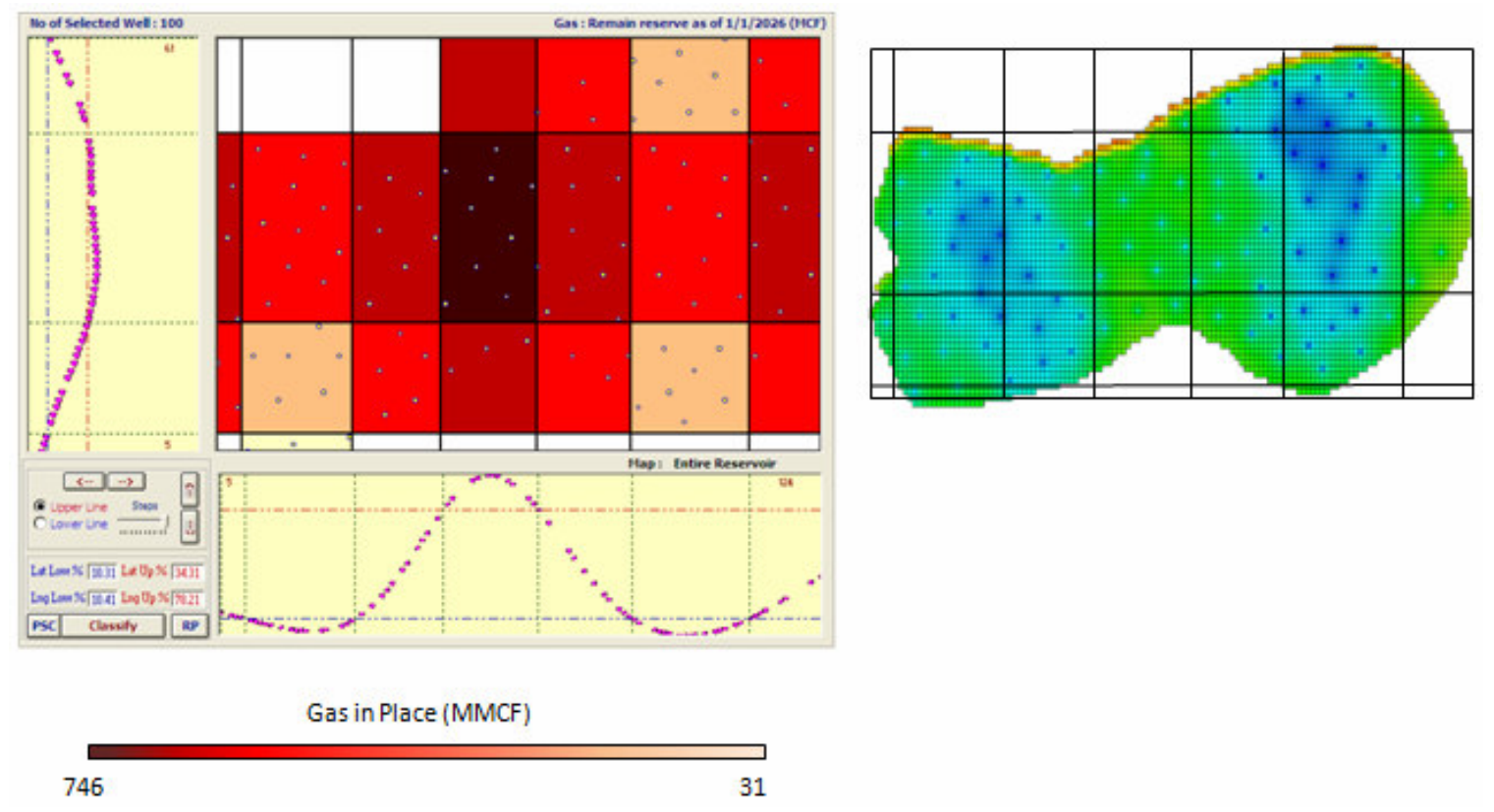

Fig. 43 - RRQI TLM-3. Gas Distribution after 26 years.

After completing these tests, important conclusions have been found:

$\checkmark$ IPDA technique recognizes sweet spots in multi-layer formations.

$\checkmark$ Using this methodology, the gas distribution may be predicted at any time in the life of a field.

III - Estimated Ultimate Recovery.

In the first set of results for Single Layer Model, it was demonstrated that the IPDA technique estimates EUR with accuracy. Now, it is necessary to determine if the same conclusion can be reached for multi-layered formations.

For the three versions of the Two-Layer Model, the 35 years EUR was calculated using the IPDA technique. Then, it was compared to the cumulative 
production at 35 years recorded in the simulator. This process was performed in a well-to-well basis. The percentage of error between the two variables was also measured.

The first case studied was the TLM-1. In this model, the EUR predicted by the IPDA technique had an average difference measured to be $5.23 \%$. The results are presented in Fig. 44. The orange zone represents the 35 years of cumulative production recorded in the simulator. On the other hand, the blue zone is the 35 years EUR calculated by the IPDA.

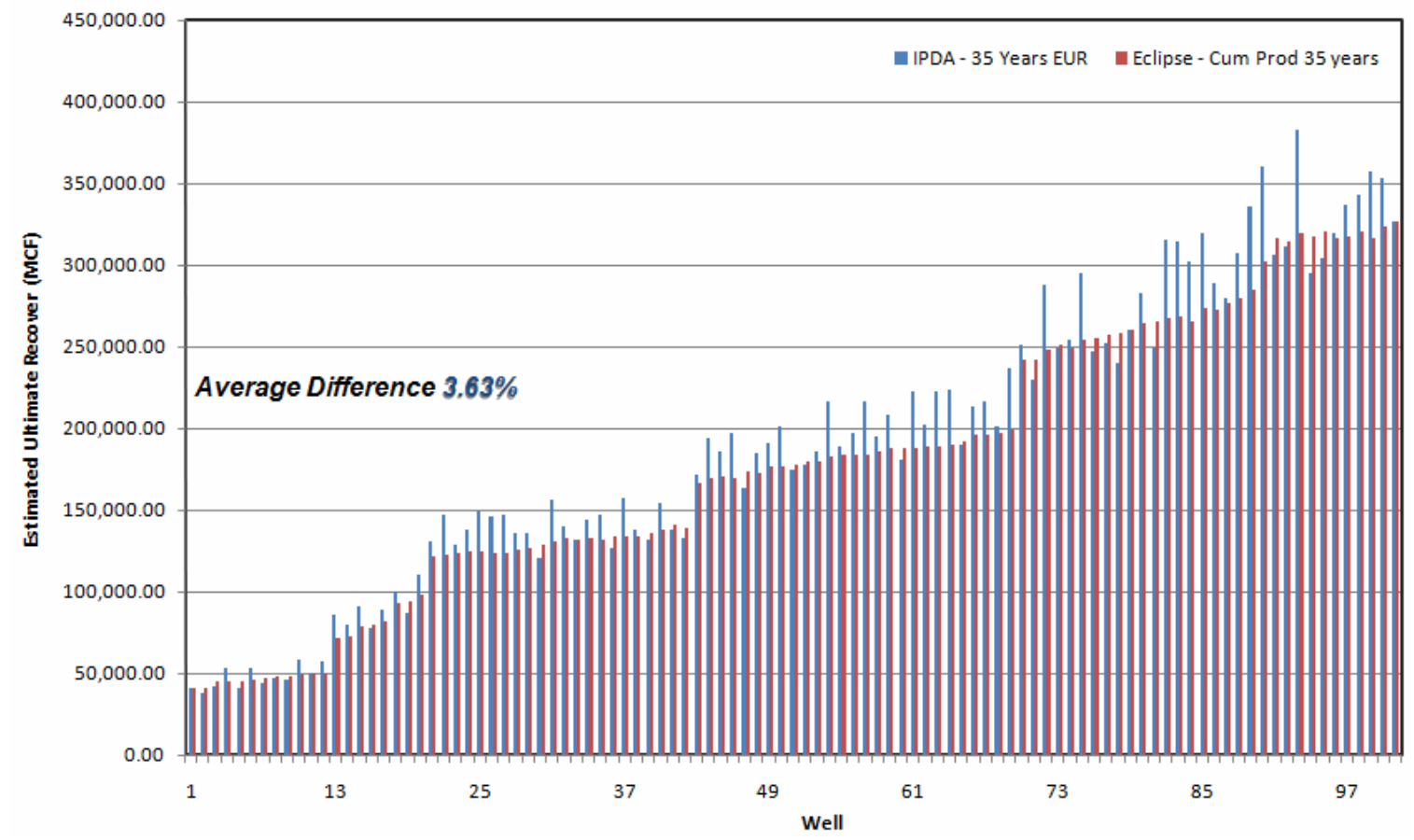

Fig. 44 - TLM-1. Evaluation of 45 years EUR.

One issue should be highlighted. The IPDA showed a tendency to overestimate EUR values in the Single Layer Model. However, in this model this factor was not present. The explanation is found in the hyperbolic index $b$. Most of the decline curves for the Two-Layer Model had $a b$ value of less than 0.5 . 
The same procedure was repeated for model TLM-2 and TLM-3 and similar results were found.

$$
\text { IV-Permeability. }
$$

In the previous sections, it has been proven that the IPDA has the ability to recognize sweet spots at any time in the life of a field. In addition, one of the most significant advantages of the Intelligent Production Data Analysis is that it estimates permeability distribution over the entire field. This section presents results found regarding permeability distribution calculated by the IPDA.

TLM -1 and TLM-3 had heterogeneous permeability distribution. In these versions, each layer has a region with higher permeability than the rest of the reservoir. Fig. 45 shows the isopermeability map.

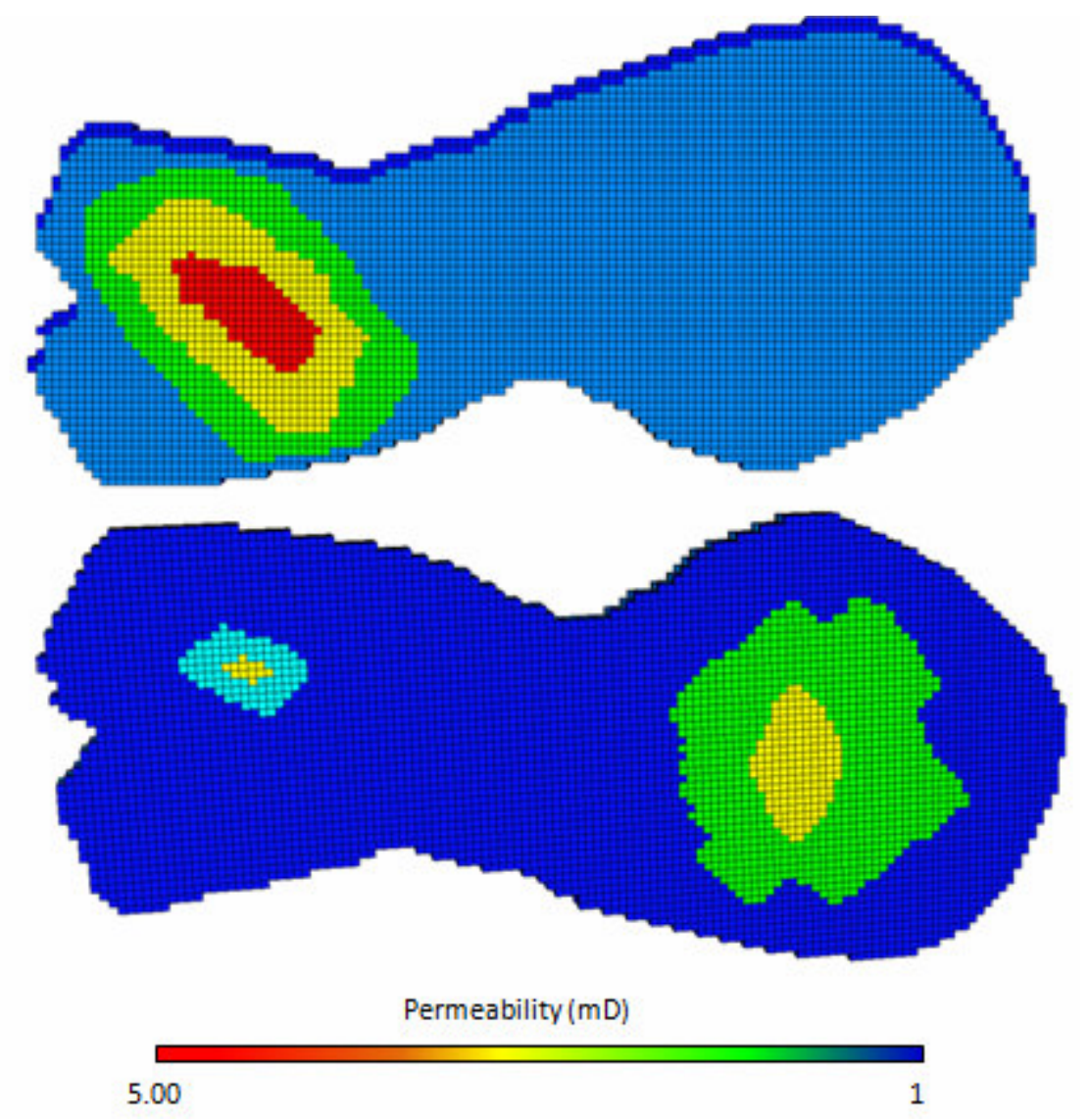

Fig. 45 - TLM-1 \& TLM-3. Actual Permeability. 
The permeability distribution calculated with the IPDA technique is shown in Fig. 46. The plot was created using XYfunction. The map indicates two zones with high permeability in the formation.

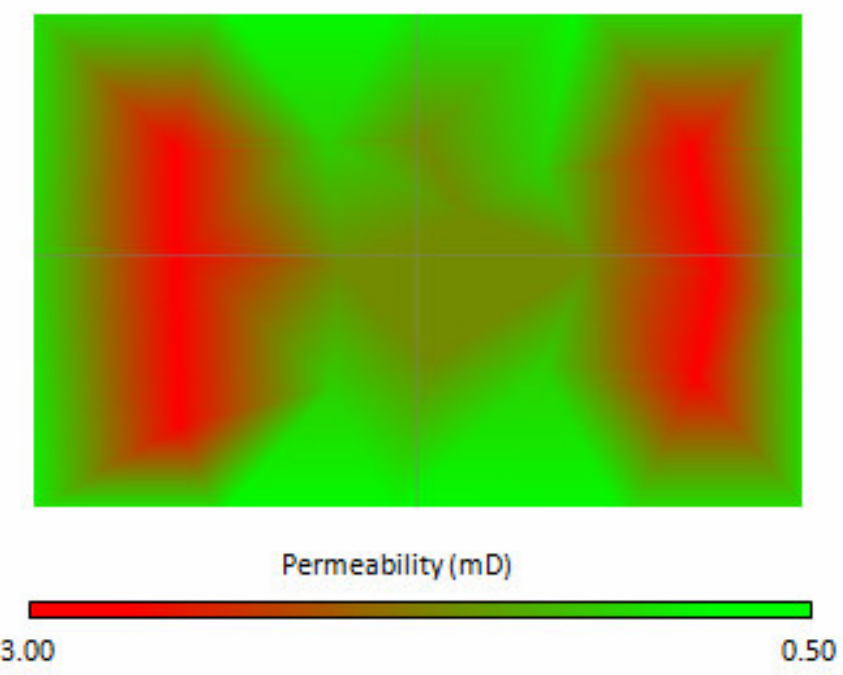

Fig. 46 - TLM-1 \& TLM-3. Permeability (IPDA).

This result makes sense, because it integrates the permeability distributions of the two layers in a single map. It proves that this methodology generates an equivalent reservoir that reproduces the behavior of the field the under evaluation.

A different result was expected in model TLM-2. In this version, the permeability distribution was homogeneous. Here, the objective was to determine if the IPDA technique detected this aspect.

The results of permeability estimation by the IPDA on TLM-2 are displayed in Fig. 47. The map reflects a quite uniform permeability distribution, with only a small alteration in one of the boundaries (which appears to be an distal point). 


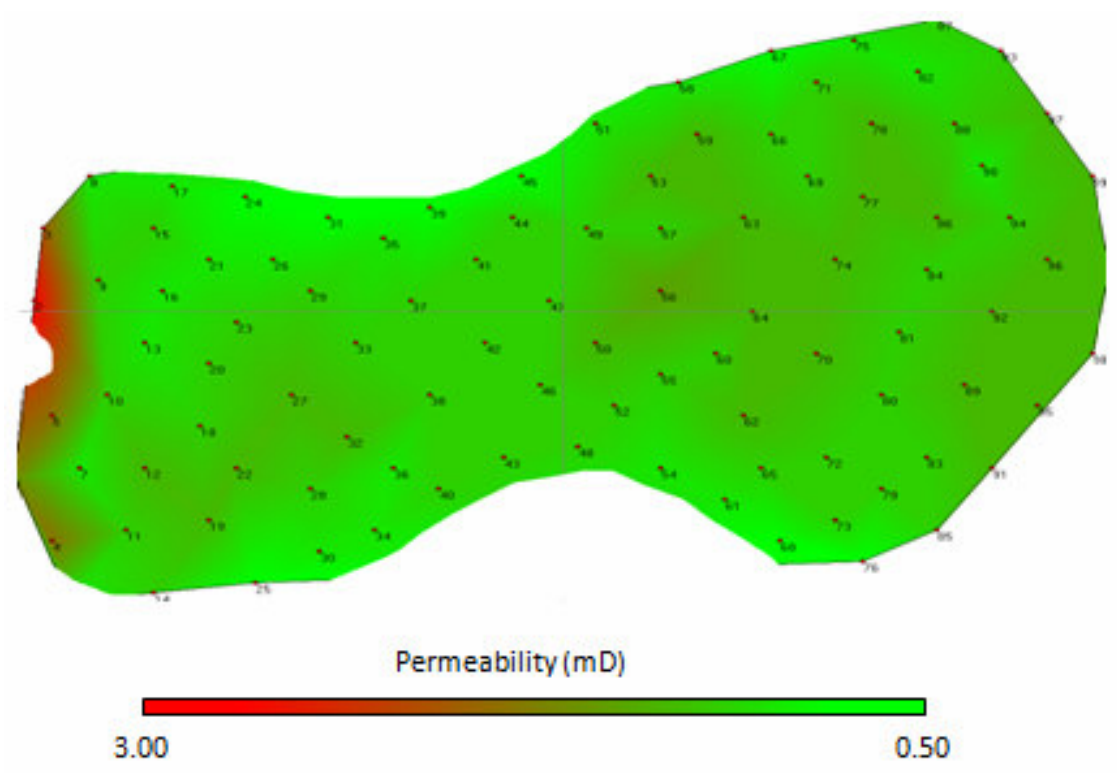

Fig. 47 - TLM-2. Permeability (IPDA).

The first set of results shows that, after comparing the results of the IPDA with the actual situation in the simulator, the IPDA technique is capable of:

$\checkmark$ Recognizing gas distribution in a formation at any given time.

$\checkmark$ Providing accurate predictions of EUR.

$\checkmark$ Estimating field-wide permeability distribution

Moreover, it has proven to work for both single and multi-layer formations. 


\subsection{Second Set of Results - Well properties}

The second set targeted the predictions of well parameters, specifically:
$\checkmark$ Fracture half-length
$\checkmark$ Drainage area.

First, the drainage area was evaluated under normal conditions (no skin). Results of drainage area distribution in SLM-100 according to the IPDA showed a uniform distribution with higher values in the lower-left area of the field. (See Fig.48)

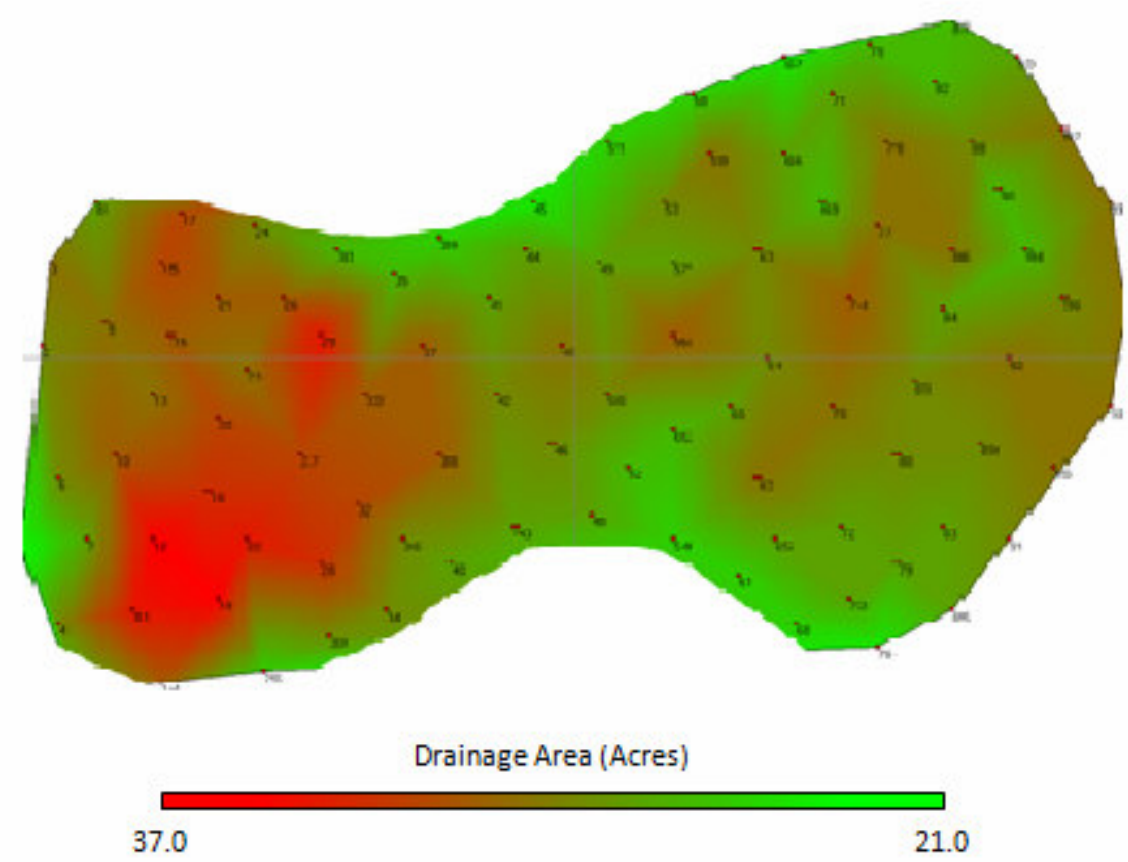

Fig. 48 - SLM 100. Drainage area (IPDA).

Then, a special routine was designed to assess the ability of the IPDA to predict alterations in the drainage area.

Positive skin factor $(+3)$ was added to wells located at the left region, for the purpose of reducing the effective radius and consequently the drainage area. 
By contrast, a negative skin (-3) was put in to the wells located at the right region of the reservoir, causing them to behave as stimulated wells.

The results of the new drainage area distribution according to the IPDA are shown in Fig. 49. In the right zone the drainage area increased significantly while in the left zone it decreased. This proves that the IPDA technique is able to identify the zone in which the field will have a higher drainage area.

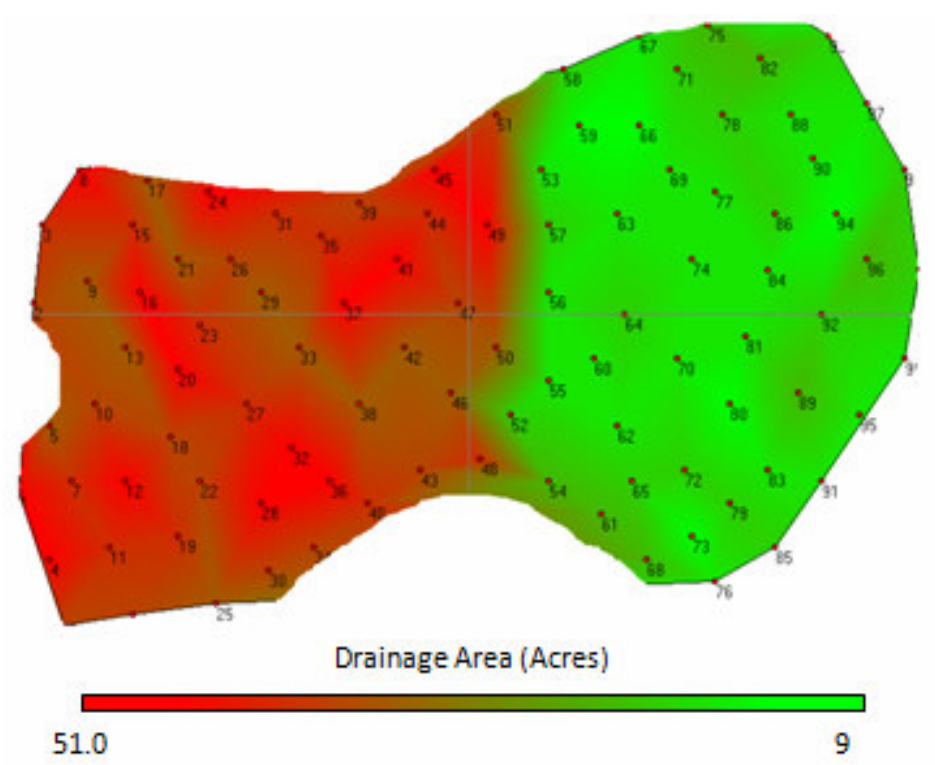

Fig. 49 - Drainage Area Distribution (with skin).

Similar results were obtained when the fracture half-length was evaluated. This demonstrates that Intelligent Production Data Analysis may be used to estimate individual well parameters in order to detect zones with poor performance. 


\subsection{Third set of results - Well Performance.}

\subsubsection{Under-performer wells}

The IPDA technique has come up with a procedure for recognizing underperformer wells. The routine monitors the behavior of every well in the reservoir. It evaluates the performance of a well as compared to its neighbors in the surrounding region.

In order to identify under-performer wells, a positive skin (+4) was added to ten wells randomly selected in model TLM 1. The high skin value affected the production capacity of these wells negatively, drastically decreasing their performance.

The results provided by the IPDA analysis are shown in Fig. 50. The wells colored blue are those that the IPDA technique predicted would be underperformers.

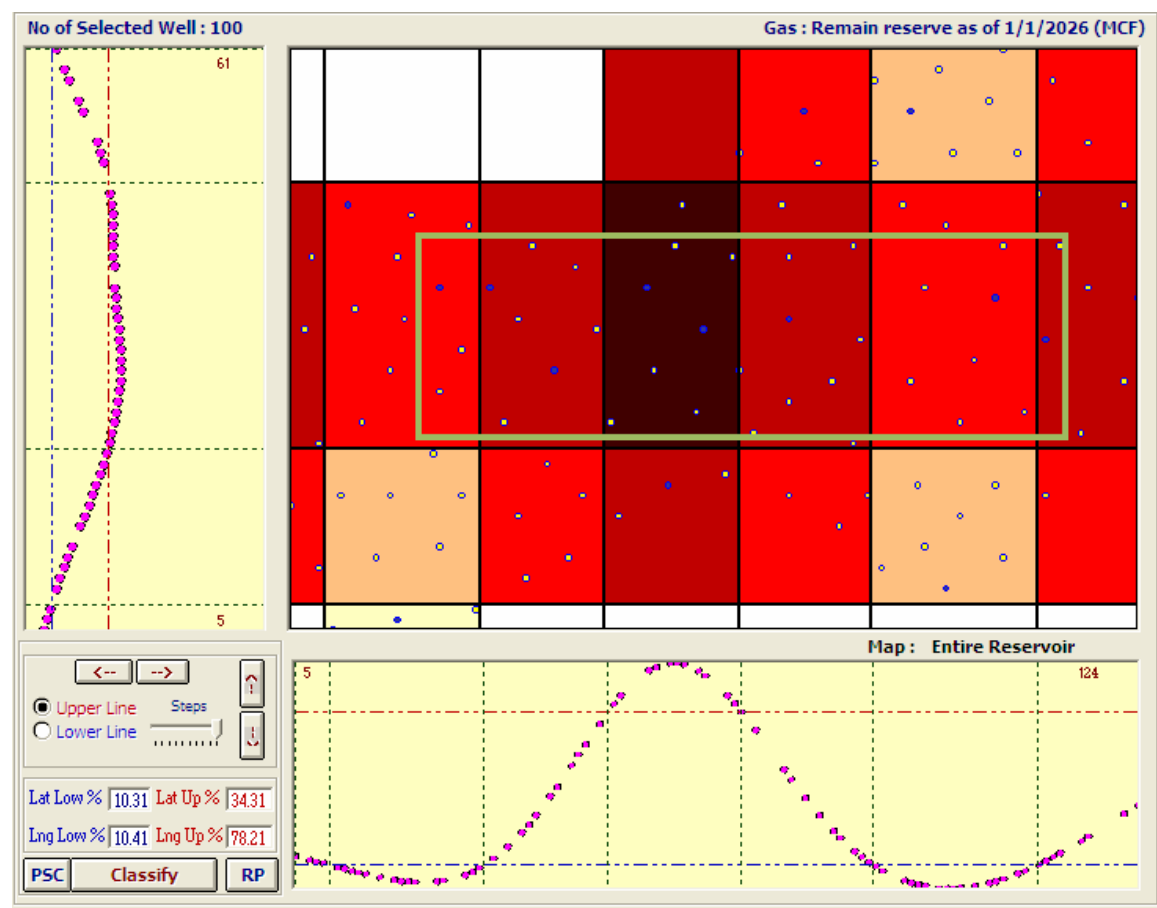


Fig. 50 - Under-Performer Wells.

The IPDA technique recognizes eight of the ten evaluated wells but marked some of the wells located at the boundaries as under-performers as well. This malfunction is due to the fact that this technique is not aware of reservoir boundaries. Normally the wells located close to a reservoir boundary experience a reduction in the potential drainage area.

For example, in Fig. 51. case (a) indicates the potential drainage area of a well when it does not reach any boundary. On the other hand, case $(b)$ illustrates the potential drainage area of this same well, but in a situation where it is located close to one of the reservoir boundaries.
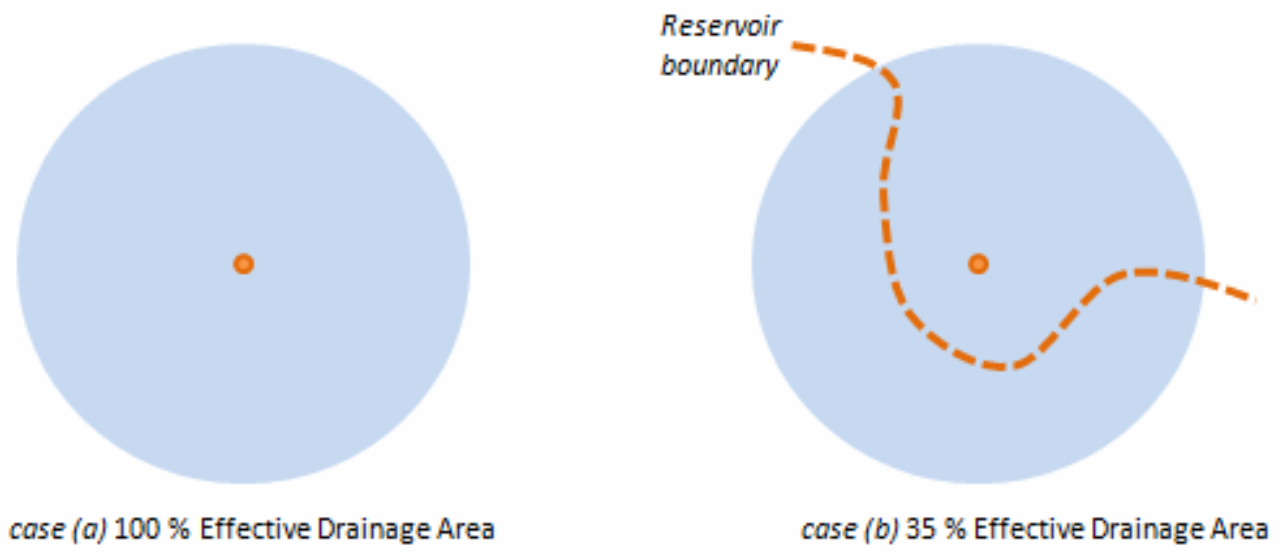

Fig. 51 - Impact of reservoir boundary on well performance.

Since the IPDA technique does not recognizes reservoir boundaries, it determines that wells located in these locations should produce at a higher capacity. Therefore, the under-performer tool selects them as wells with poor productivity. 


\subsubsection{Evaluation of "What-if Scenarios"}

In the SLM-100 Model, 100 wells are uniformly distributed along the reservoir (See Fig. 52). Clearly, there are areas that have enough separation and represent an excellent choice for infill drilling. What is to be demonstrated is that this methodology is capable of modeling the behavior of future wells in advance.

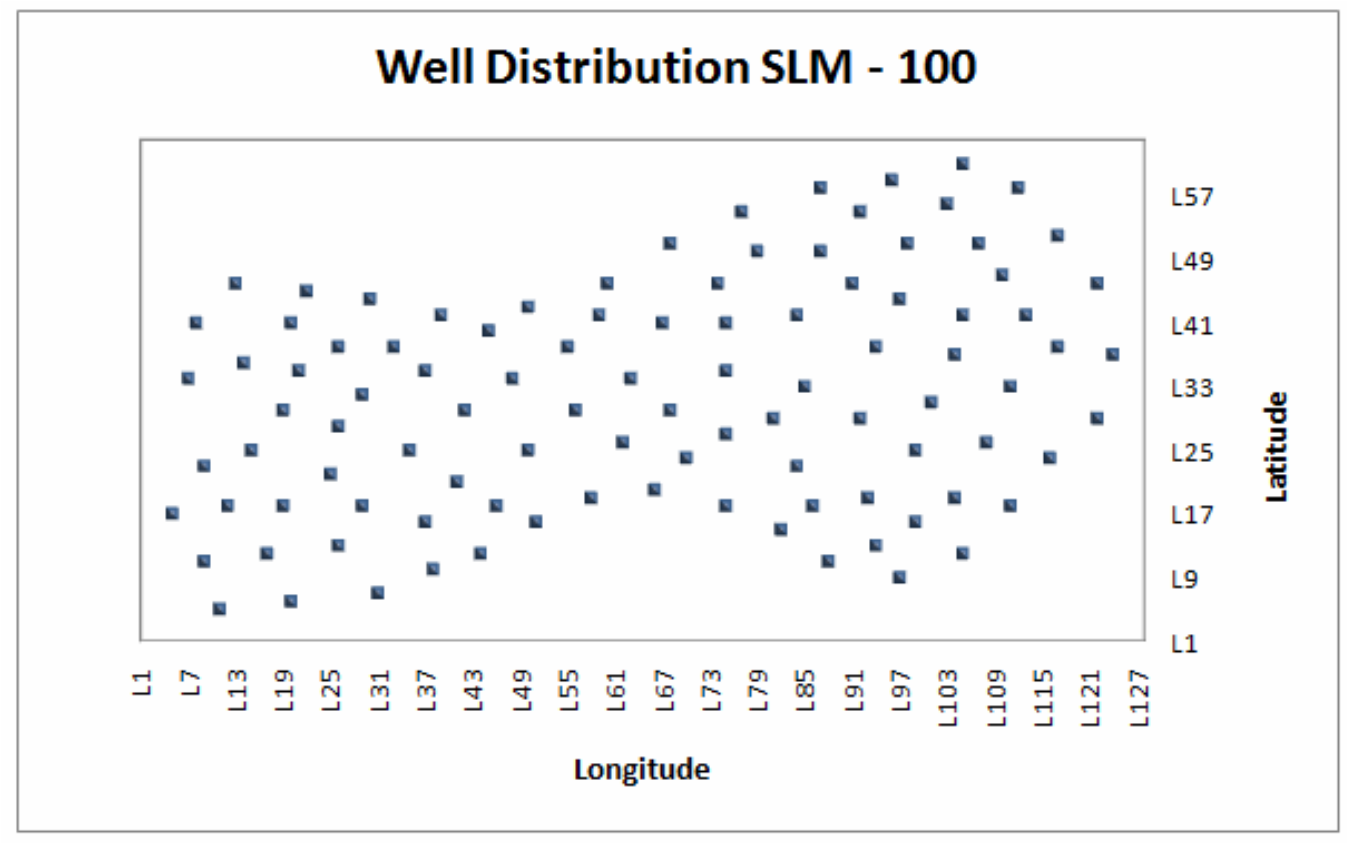

Fig. 52 - SLM 100. Well Distribution.

In Fig. 53 the red dots represent the location selected for infill drilling. In this case, some wells are located close to the boundaries (green zone) while the others are in the mid-region of the reservoir (red zone). 


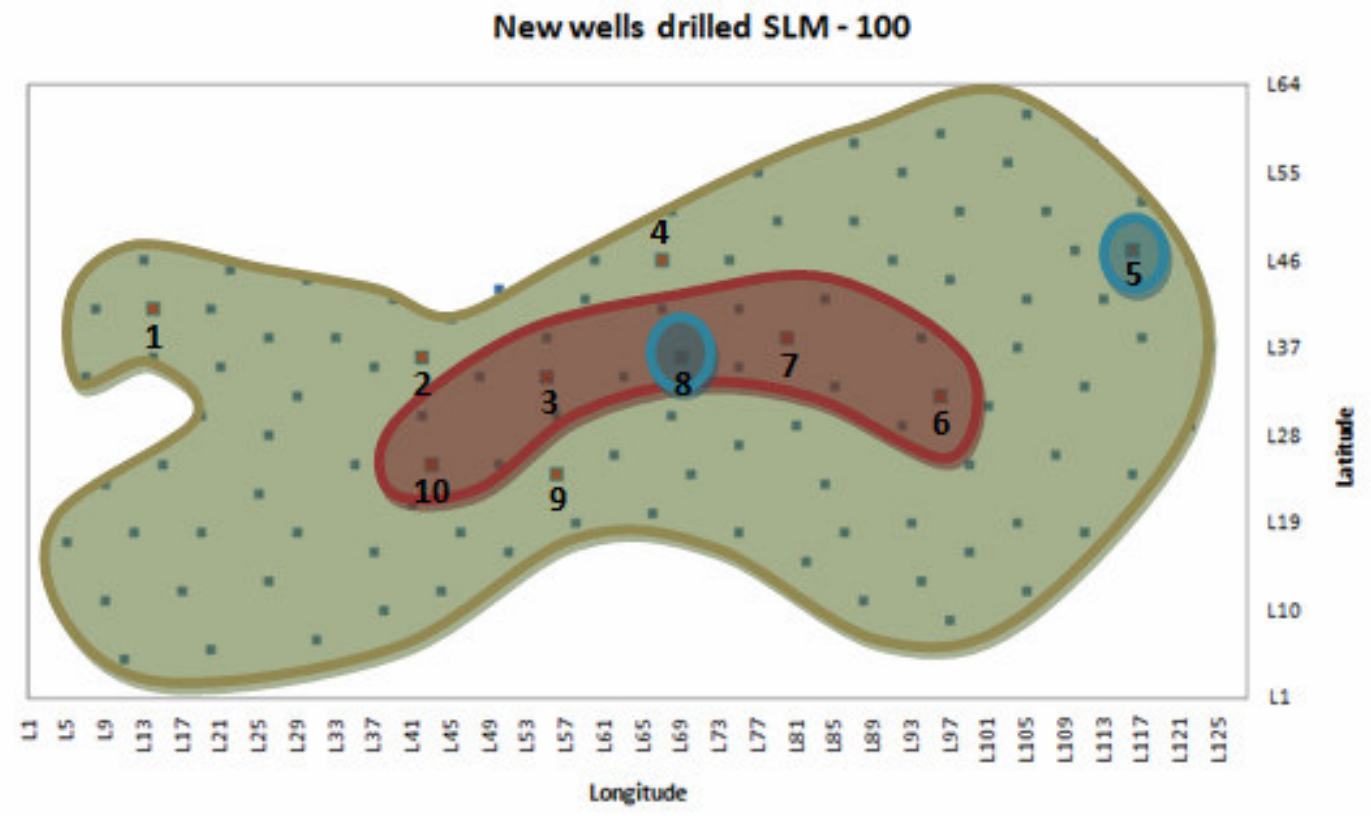

Fig. 53 - Locations selected for infill drilling.

Using the what-if scenarios tool, the decline parameters (Qi, Di and b) were estimated. Then the decline curves of these wells were reproduced.

To assess the accuracy of the what-if scenarios feature, it was necessary to drill wells in the simulator, using exactly the same locations indicated in Fig. 53. After that, the results of the simulator were compared to the predicted decline curves.

Results indicated that the wells located close to the boundaries (Green Zone) did not reproduce the behavior predicted by the IPDA. On the other hand, the production of the wells located in the red zone (separated from the boundaries) had a notable similarity to the decline curve modeled with the what-if scenarios tool.

In order to understand the aforementioned situation, the behavior of two wells was evaluated in detail. One of them was located close to the boundary while the other one was drilled in the mid-region of the reservoir (See in Fig.53 the wells encircled in blue). 
In addition, Net Present Value (NPV) was employed in order to assess the economic impact.

Case I - Well separated to the boundary

Fig. 54 is a plot of gas production rate vs. time for Well \# 8. In this case, the production from the simulator (light orange) follows the same trend as the decline curve modeled using what-if scenarios (dark orange).

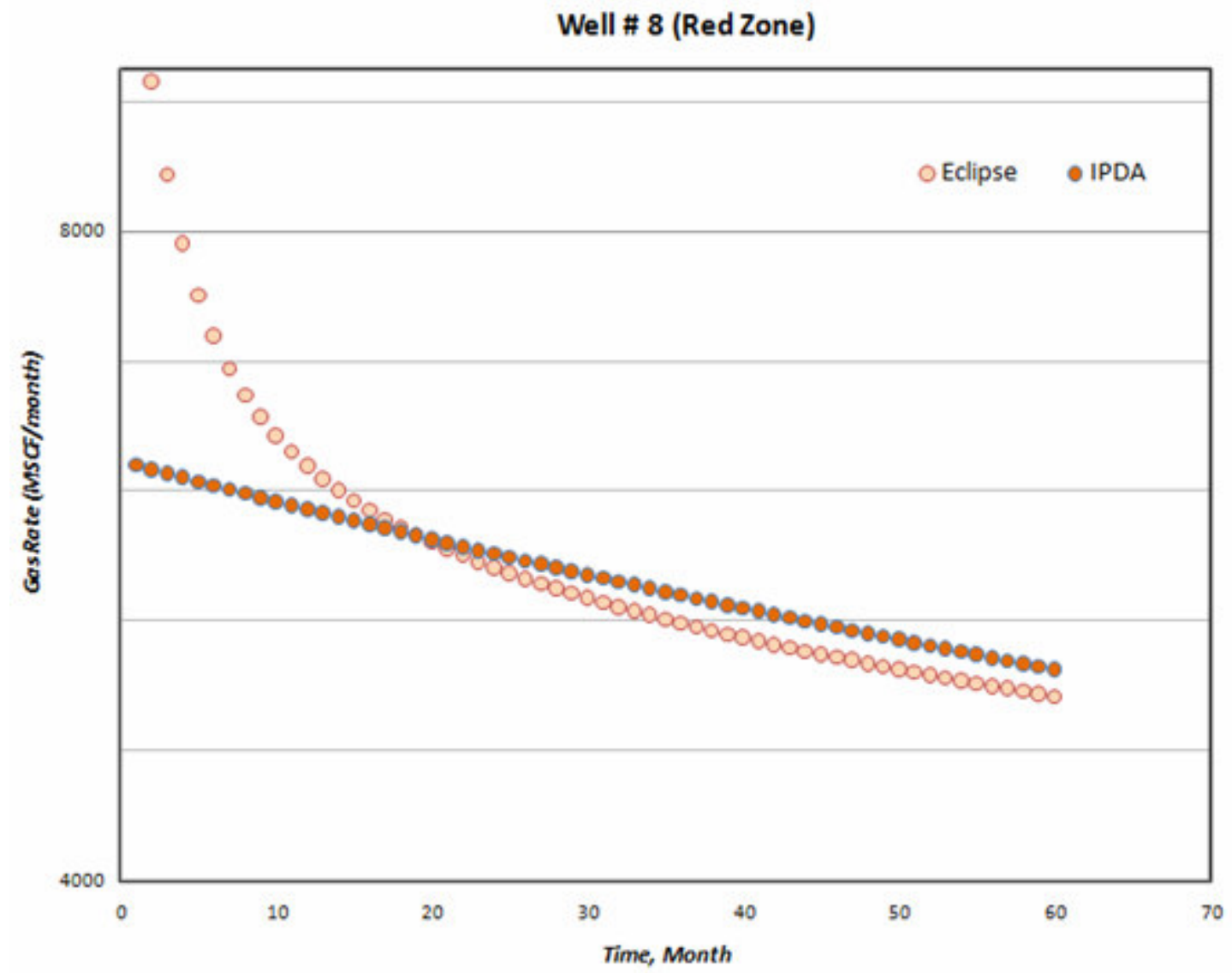

Fig. 54 - Gas Flow Rate vs. Time Well \# 8.

An economic analysis was performed on this well and the following conditions were assumed: 
$\checkmark$ Interest Rate: $8 \%$

$\checkmark$ Gas Price: $\$ 7.00$

$\checkmark$ Operation costs: $0.50 \$ / M C F$

$\checkmark$ Drilling cost: $\$ 200,000.00$

\section{Economic Analysis on Well \# 8}

\begin{tabular}{|c|c|}
\hline Qi & 6584 \\
\hline Di & 0.0039 \\
\hline$b$ & 0.7 \\
\hline Interest & $10 \%$ \\
\hline Gas Price & 7.00 \\
\hline Op. Cost & $\$ \quad 0.50$ \\
\hline Drill. Cost & $\$ 200,000.00$ \\
\hline
\end{tabular}

\begin{tabular}{|c|c|c|c|c|c|c|c|c|c|}
\hline \multicolumn{10}{|c|}{ Economic Analysis on Well \# 8 (based on IPDA) } \\
\hline Year & Production & Ope & ation Costs & & Revenue & & Cash Flow & & Present Value \\
\hline 1 & $77,058.91$ & $\$$ & $38,529.45$ & $\$$ & $539,412.35$ & $\$$ & $300,882.90$ & $\$$ & \\
\hline 2 & & $\$$ & & $\$$ & & $\$$ & & & \\
\hline 3 & & $\$$ & & $\$$ & & $\$$ & & & \\
\hline 4 & & $\$$ & 4.52 & $\$$ & 3.29 & $\$$ & 68.77 & sta & 9.88 \\
\hline 5 & $64,813.64$ & $\$$ & $32,406.82$ & $\$$ & $453,695.47$ & $\$$ & $421,288.65$ & $\$$ & $261,587.11$ \\
\hline
\end{tabular}

$\$ \quad 1,574,863.44$

Difference Between methods

Ammount Percentage

\begin{tabular}{|c|c|c|c|c|c|c|c|c|c|}
\hline \multicolumn{10}{|c|}{ Economic Analysis on Well \# 8 (actual situation) } \\
\hline Year & Production & Ope & ation Costs & & Revenue & $\mathrm{Ne}$ & t Cash Flow & Net & resent Value \\
\hline 1 & $90,798.16$ & $\$$ & $45,399.08$ & $\$$ & $635,587.15$ & $\$$ & $390,188.07$ & $\$$ & $354,716.42$ \\
\hline 2 & 050.68 & $\$$ & 7.023 .3 & $\$$ & 4.73 & $\$$ & & $\$$ & .89 \\
\hline 3 & 786.74 & $\$$ & $34,393.37$ & $\$$ & 07.18 & $\$$ & 447, & $\$$ & 3.22 \\
\hline 4 & 63.39 & $\$$ & $32,681.70$ & $\$$ & 3.73 & $\$$ & 424, & $\$$ & 186.49 \\
\hline 5 & $62,704.37$ & $\$$ & $31,352.19$ & $\$$ & $438,930.59$ & $\$$ & $407,578.41$ & $\$$ & $253,074.12$ \\
\hline
\end{tabular}

$\$ 1,631,693.14$

Table 6 - Economic analysis on well \# 8.

Table 6 summarizes the results of the economic analysis performed on well \# 8. IPDA technique estimated the NPV of this well as $\$ 1,574,863$. The actual NPV of the well was $\$ 1,631,693$.

Case II - Well close to the boundary

Fig. 55 is a plot of gas production rate vs. time for Well \# 5. In this case, the production from the simulator (light orange) does not follow a similar trend as the decline curve modeled using what-if scenarios (dark orange). In this case, the prediction made by the IPDA overestimated the well production. 


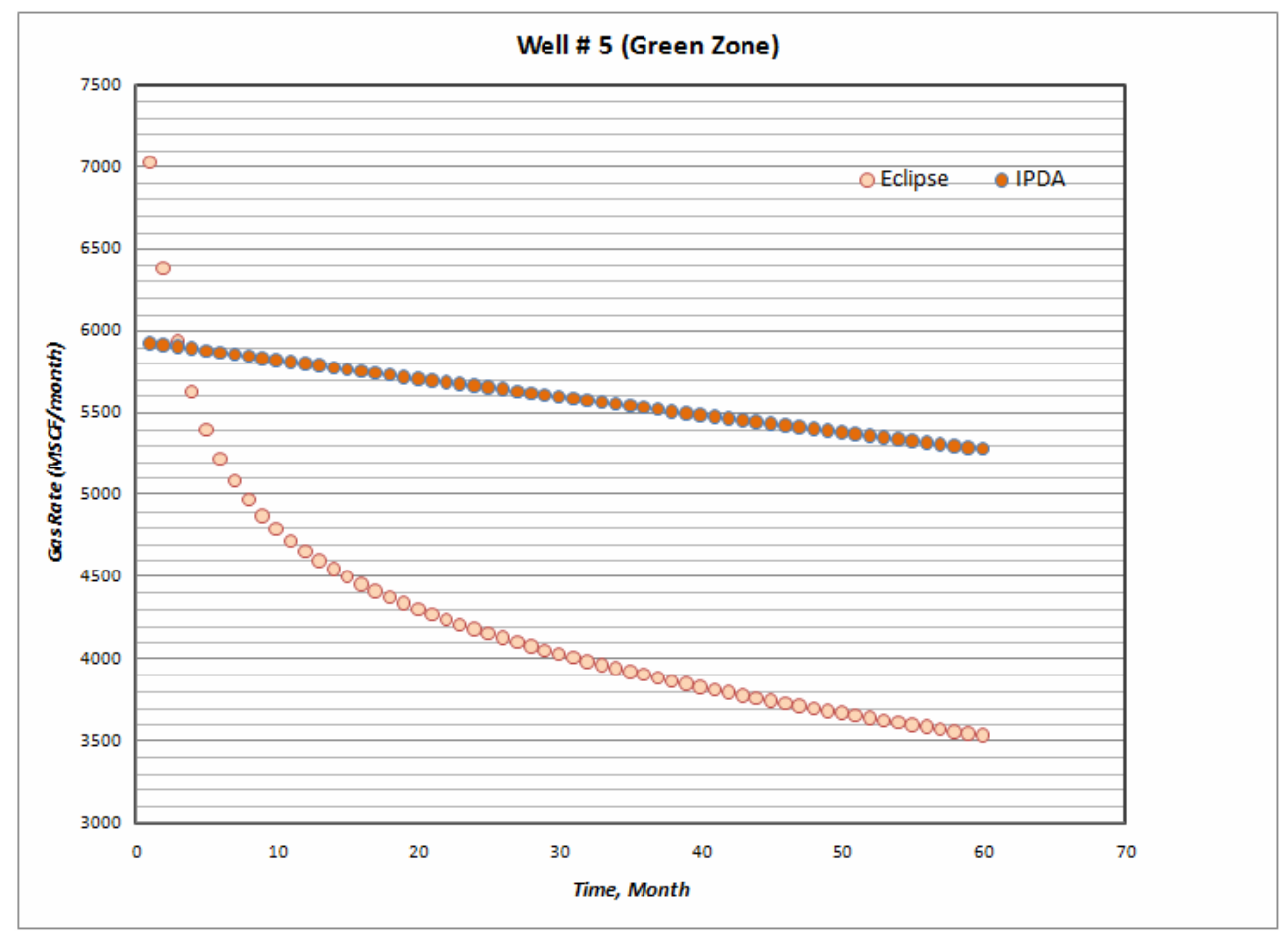

Fig. 55 - Gas flow rate vs. time well \# 5.

An economic analysis was performed on this well assuming the same conditions selected for case $I$.

Economic Analysis on Well \# 5

\begin{tabular}{|c|c|c|}
\hline Qi & & 6428 \\
\hline Di & & 0.0031 \\
\hline$b$ & & 0.7 \\
\hline Interest & & $10 \%$ \\
\hline Gas Price & $\$$ & 7.00 \\
\hline Op. Cost & $\$$ & 0.50 \\
\hline Drill. Cost & $\$$ & $200,000.00$ \\
\hline
\end{tabular}

\begin{tabular}{|c|c|c|c|c|c|c|c|c|c|}
\hline \multicolumn{10}{|c|}{ Economic Analysis on Well \# 8 (based on IPDA) } \\
\hline Year & Production & $O p$ & ration Costs & & Revenue & & t Cash Flow & & resent Value \\
\hline 1 & $70,351.98$ & $\$$ & $35,175.99$ & $\$$ & $492,463.86$ & $\$$ & $257,287.87$ & $\$$ & $233,898.06$ \\
\hline 2 & & $\$$ & & $\$$ & & $\$$ & & . & \\
\hline 3 & & $\$$ & 549.09 & $\$$ & 7.22 & $\$$ & 8.14 & $s$ & 7.04 \\
\hline 4 & $65,548.31$ & $\$$ & $32,774.15$ & $\$$ & $458,838.14$ & $\$$ & $426,063.98$ & $\$$ & $291,007.43$ \\
\hline 5 & $64,047.07$ & $\$$ & $32,023.53$ & $\$$ & $448,329.46$ & $\$$ & $416,305.92$ & $\$$ & $258,493.23$ \\
\hline
\end{tabular}

$\$ \quad 1,480,118.32$

\begin{tabular}{cr}
\hline \multicolumn{2}{c}{ Difference Between methods } \\
\hline Ammount & Percentage \\
\hline$\$ 385,845.92$ & $26 \%$ \\
\hline
\end{tabular}

\begin{tabular}{|c|c|c|c|c|c|c|c|c|c|}
\hline \multicolumn{10}{|c|}{ Economic Analysis on Well \# 8 (actual situation) } \\
\hline Year & Production & Ope & ration Costs & & Revenue & & Cash Flow & $\mathrm{Net}$ & t Value \\
\hline 1 & $64,679.09$ & $\$$ & $32,339.55$ & $\$$ & $452,753.63$ & $\$$ & $220,414.09$ & $\$$ & $200,376.44$ \\
\hline 2 & 39.99 & $\$$ & $26,220.00$ & $\$$ & & \& & & & 2.43 \\
\hline 3 & $3,272.19$ & $\$$ & $24,136.10$ & $\$$ & $337,905.33$ & $\$$ & 9.24 & $\$$ & 39.47 \\
\hline 4 & 6.93 & $\$$ & $22,728.47$ & $\$$ & 8.51 & $\$$ & 0.05 & $\$$ & 10.02 \\
\hline 5 & $43,271.69$ & $\$$ & $21,635.85$ & $\$$ & $302,901.83$ & $\$$ & $281,265.99$ & $\$$ & $174,644.05$ \\
\hline
\end{tabular}

$\$ 1,094,272.40$

Table 7 - Economic analysis on well \# 5. 
Table 7 summarizes the results of the economic analysis performed on well \# 5. IPDA technique estimated the NPV of this well as $\$ 1,480,118$. The actual NPV of the well was $\$ 1,094,272$.

After presenting the findings for the third set of results, the following statements have been demonstrated:

$\checkmark$ The IPDA technique successfully recognizes under-performer wells in a reservoir.

$\checkmark$ The IPDA technique is able to predict decline parameters, which may be used to assess potential locations for infill drilling.

$\checkmark$ Both under-performer wells and what-if scenarios tools provide incorrect recommendations when the well under evaluation is located close to reservoir boundaries. 


\section{Chapter V-Conclusions and Recommendations}

The findings of this study are the result of a thorough and comprehensive effort. The results achieved required constructing 13 reservoirs models and performing decline curve analysis, type curve matching and history matching over approximately 1600 wells.

This study verifies that Intelligent Production Data Analysis:

1. Identifies sweet spots in a reservoir at any time.

2. Provides unified set of reservoir characteristics (initial gas distribution, permeability, etc).

3. May be applied in both single and multi-layer formations.

4. Estimates accurately the distribution of the remaining reserves at any time.

5. Forecasts Estimated Ultimate Recovery with precision.

6. Provides reliable information regarding well parameters (drainage area, fracture half-length, etc)

7. Identifies under-performer wells.

8. Assesses potential locations for infill drilling.

The following limitations were found:

1. The accuracy of the recommendations depends on the quality of the conventional analysis performed (decline curve analysis, type curve matching and history matching). Therefore, the technique provides better results once the condition of pseudo-steady state has been reached.

2. Intelligent Production Data Analysis stands for a qualitative analysis of the reservoir. Therefore, the figures estimated are not necessarily correct. 
3. The technique might provide incorrect recommendations for those wells located near to the reservoir boundaries.

4. In some cases, is not possible to find an appropriate partitioning for delineating the Relative Reservoir Quality Index (RRQI map). This situation impedes the visualization of the results. 


\section{References}

1. Arps, J.J.: "Analysis of Decline Curves," Trans., AIME (1945).

2. Fetkovitch, M.J.: "Decline Curve Analysis Using Type Curves," JPT (June 1980) 1065-1077.

3. Anderson D.M., Sttots G., Mattar L.:"Production Data Analysis Challeges, Pitfalls, Diagnostics“. SPE 102048.

4. Poe Jr. B.D., Zheng-Poe A., Boney C.L.:"Production Data Analysis and forecasting using a comprehensive analysis system". SPE 52178.

5. Agarwal R.G., et al: "Analyzing Well Production Data Using CombinedType-Curve and Decline-Curve-Analysis Concepts," SPEREE. October 1999. 475-479.

6. Poe Jr. B.D., Conge J.G., Farkas R., et al.: "Advanced fracture well diagnostic for production data analysis“. SPE 62881.

7. Mohaghegh S.D., Gaskari R. and Jalali J.: "New Method for Production Data Analysis to Identify New Opportunities in Mature Fields: Methodology and Application". SPE 98010.

8. Jalali J., Mohaghegh S., Gaskari, R.: "Identifying Infill Locations and Underperformer Wells in Mature Fields using Monthly Production Rate Data, Carthage Field, Cotton Valley Formation, Texas" SPE 104550.

9. Gaskari R., Mohaghegh S., Jalali, J. "An integrated technique for production data analysis with applications to mature fields". SPE 100562.

10. Cox D.O., Hansen J.T.: „Advanced type curve analysis for low permeability gas reservoirs“ SPE 35595.

11. Newman D.: "Engineering Economic Analysis". Trans. 271.

12. Ertekin T., Abou- Kassem J., King.: "Basic Applied Reservoir Simulation". SPE Textbook Series Vol. 7. 9-11.

13. Advanced Type Curve Analysis for Low Permeability Gas Reservoirs", Cox, Kuuskraa and Hansen. SPE 35595, 1998. 


\section{Appendix A}

Single Layer Model - Structural Maps 


\section{Single Layer Model - Isopach Map}

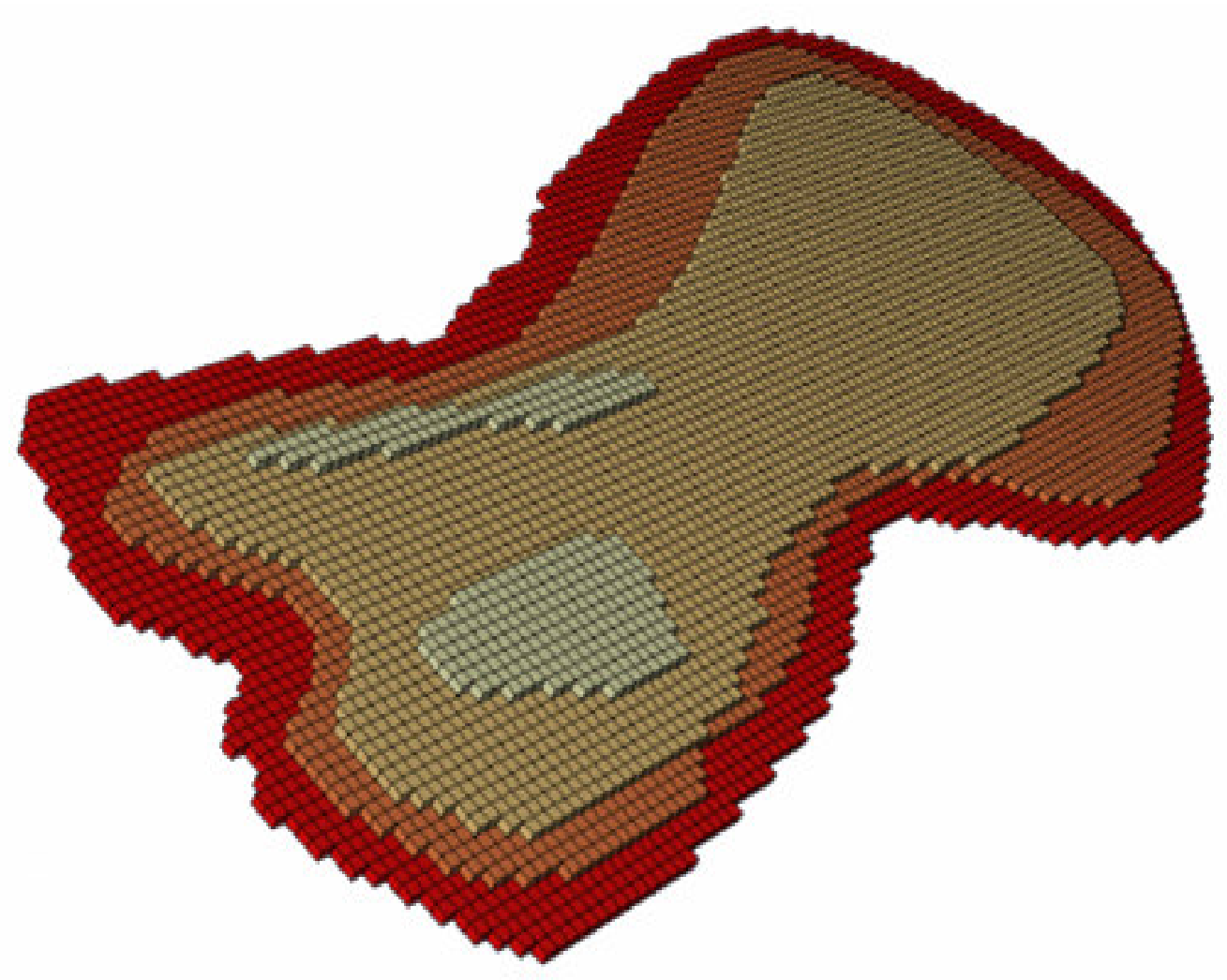

Thickness (ft) 
Single Layer Model - Isopermeability Map

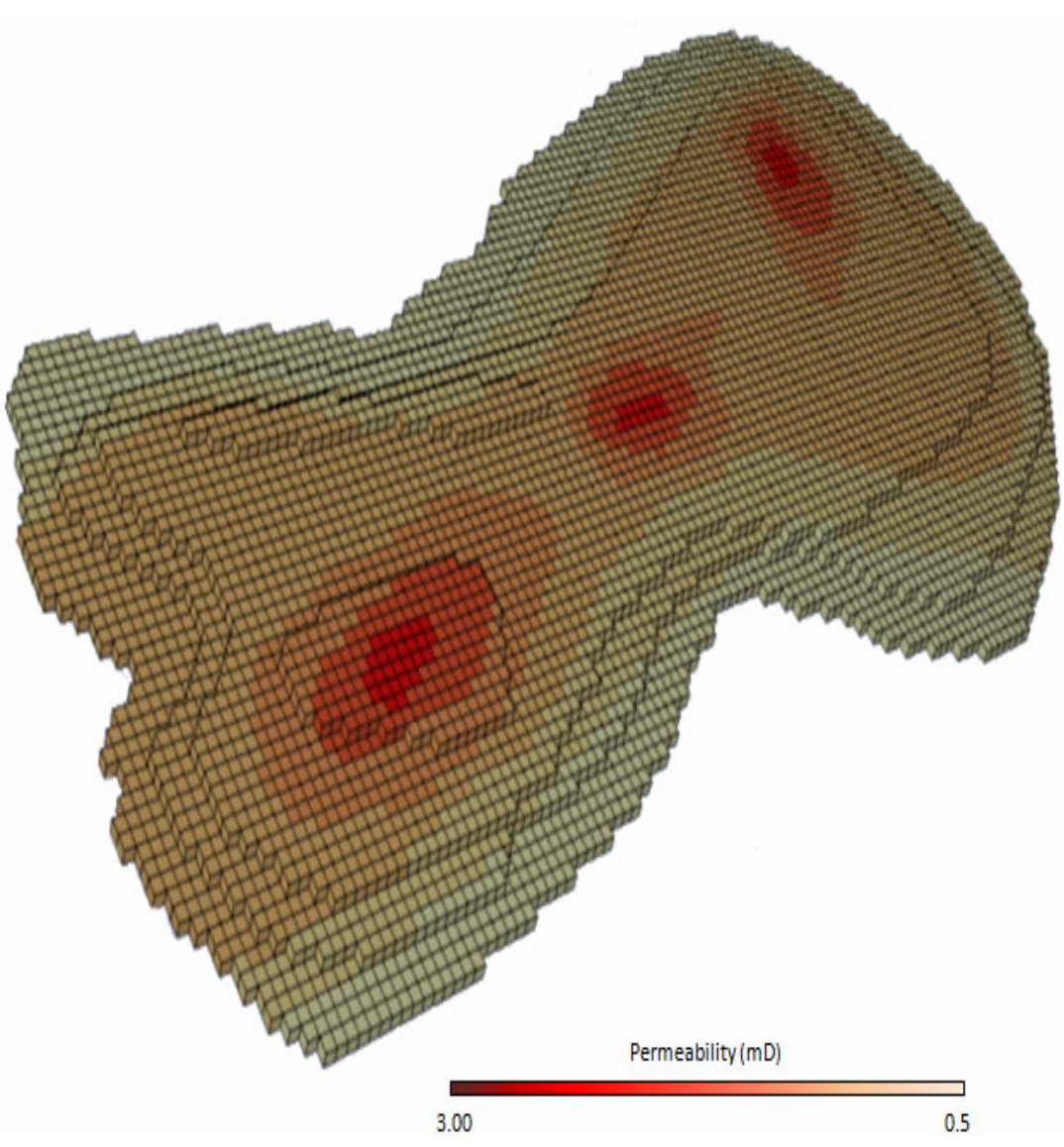




\section{Single Layer Model - Isoporosity Map}

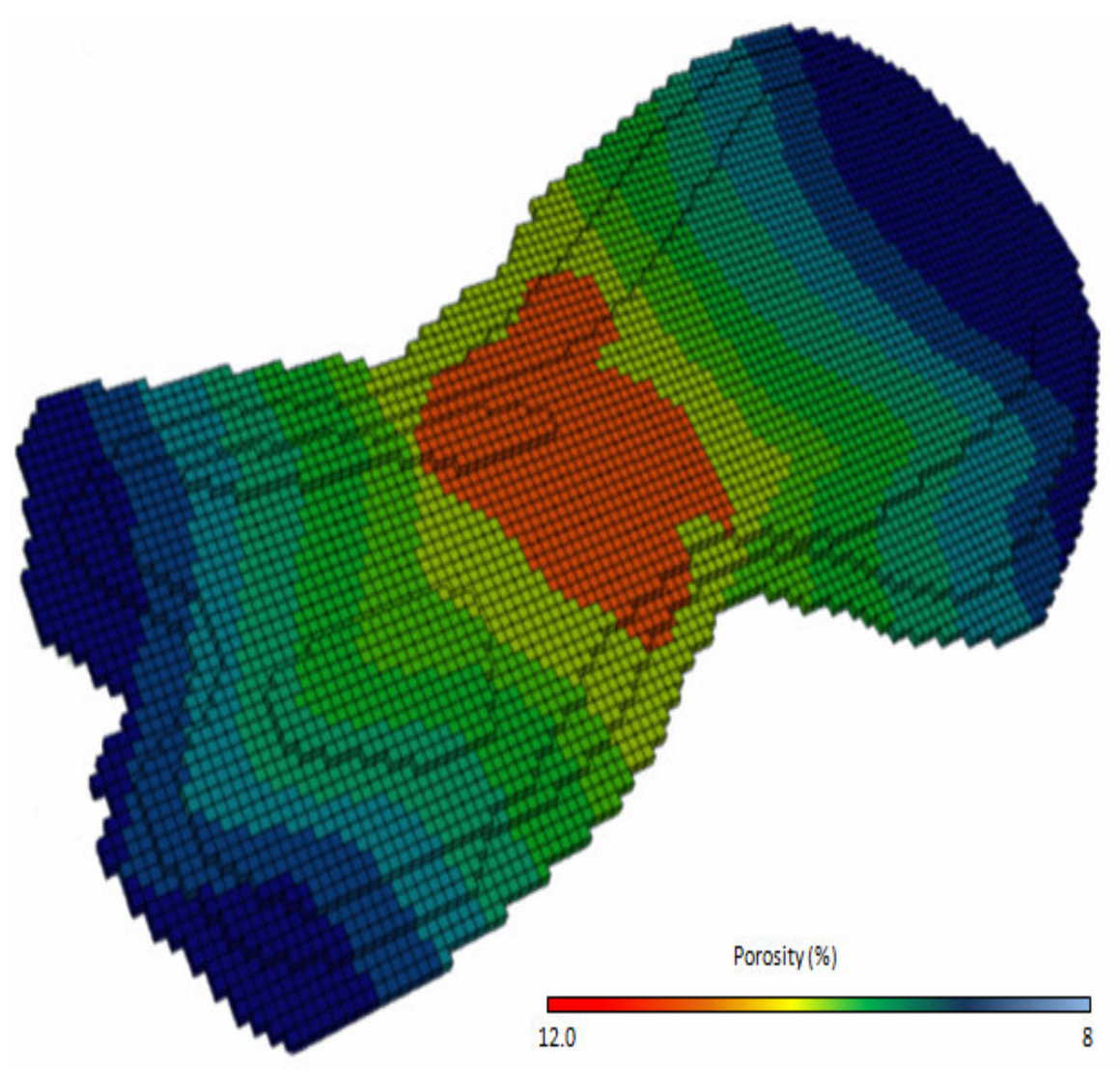




\section{Appendix B}

Two-Layer Model - Structural Maps 
Two-Layer Model - Isopermeability Map

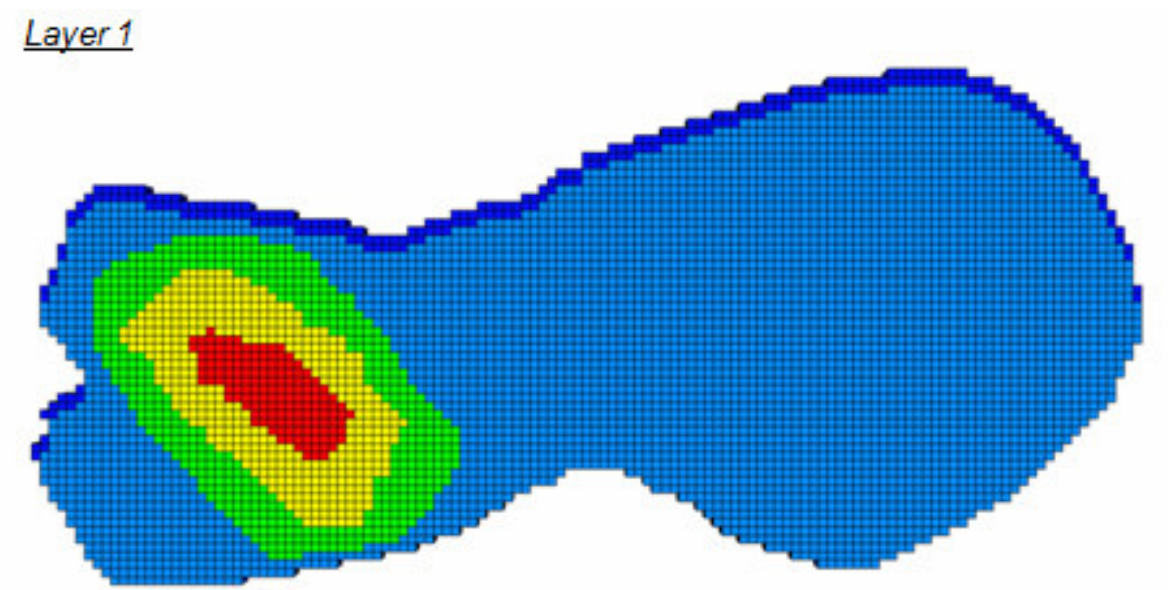

Layer 2

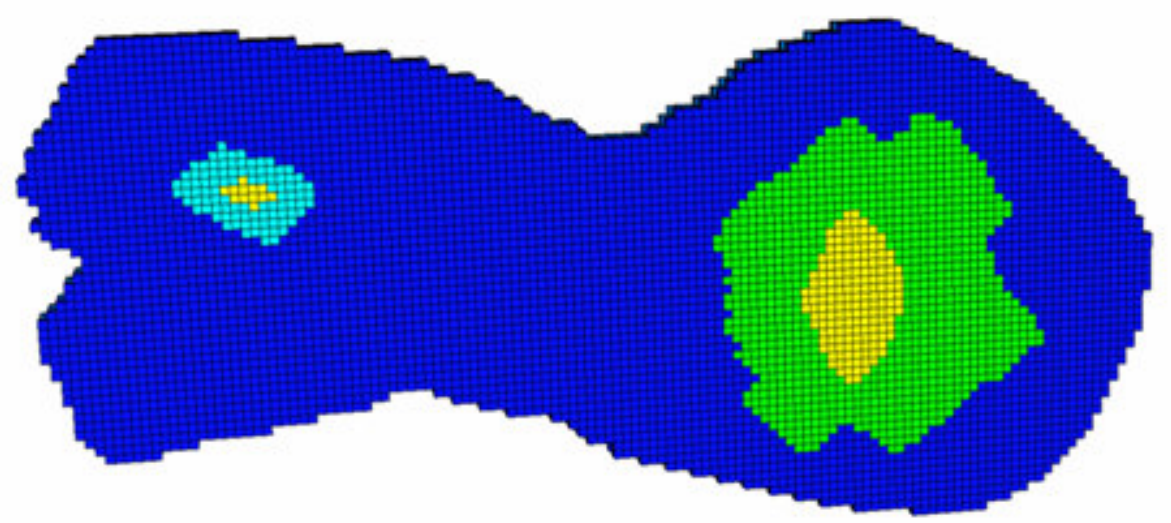

Permeability $(\mathrm{mD})$

5.00

1.00 
Two-Layer Model - Isoporosity Map
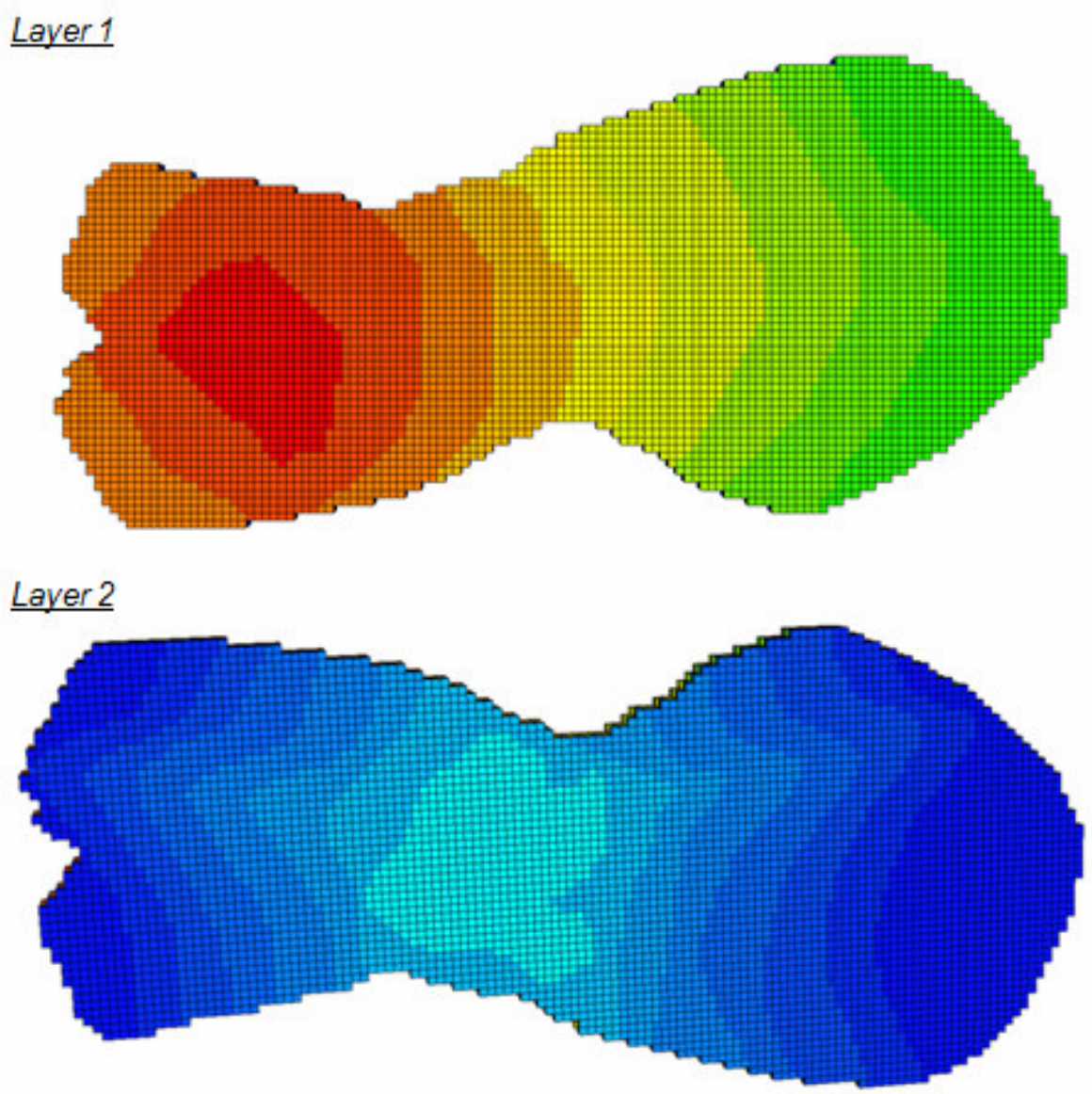

Porosity (\%)

25.0 


\section{Appendix C}

Source code of the program developed to rearrange production data in order to make it compatible with IPDA.

Language: Visual Basic. Application: Microsoft Excel. 


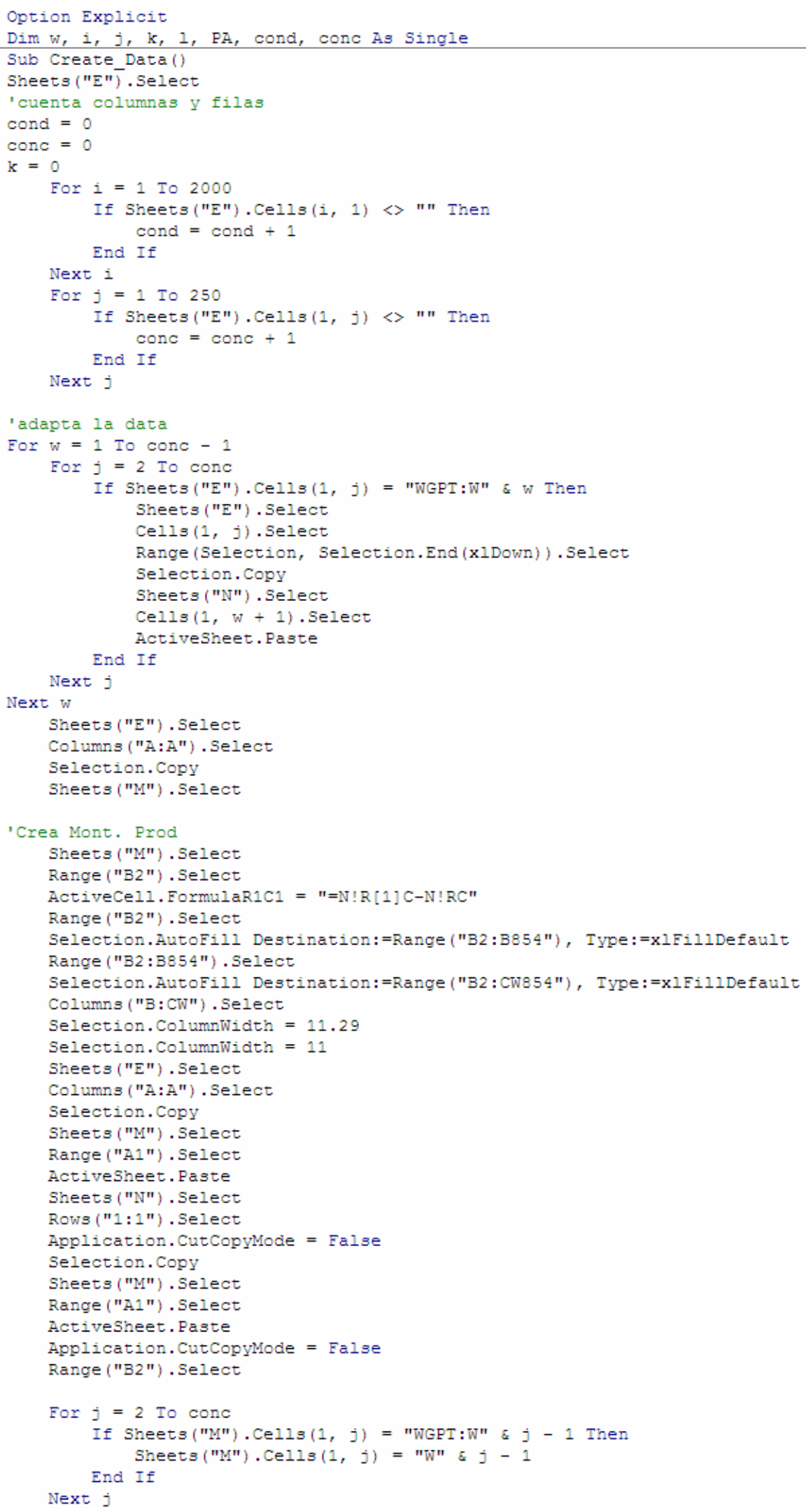




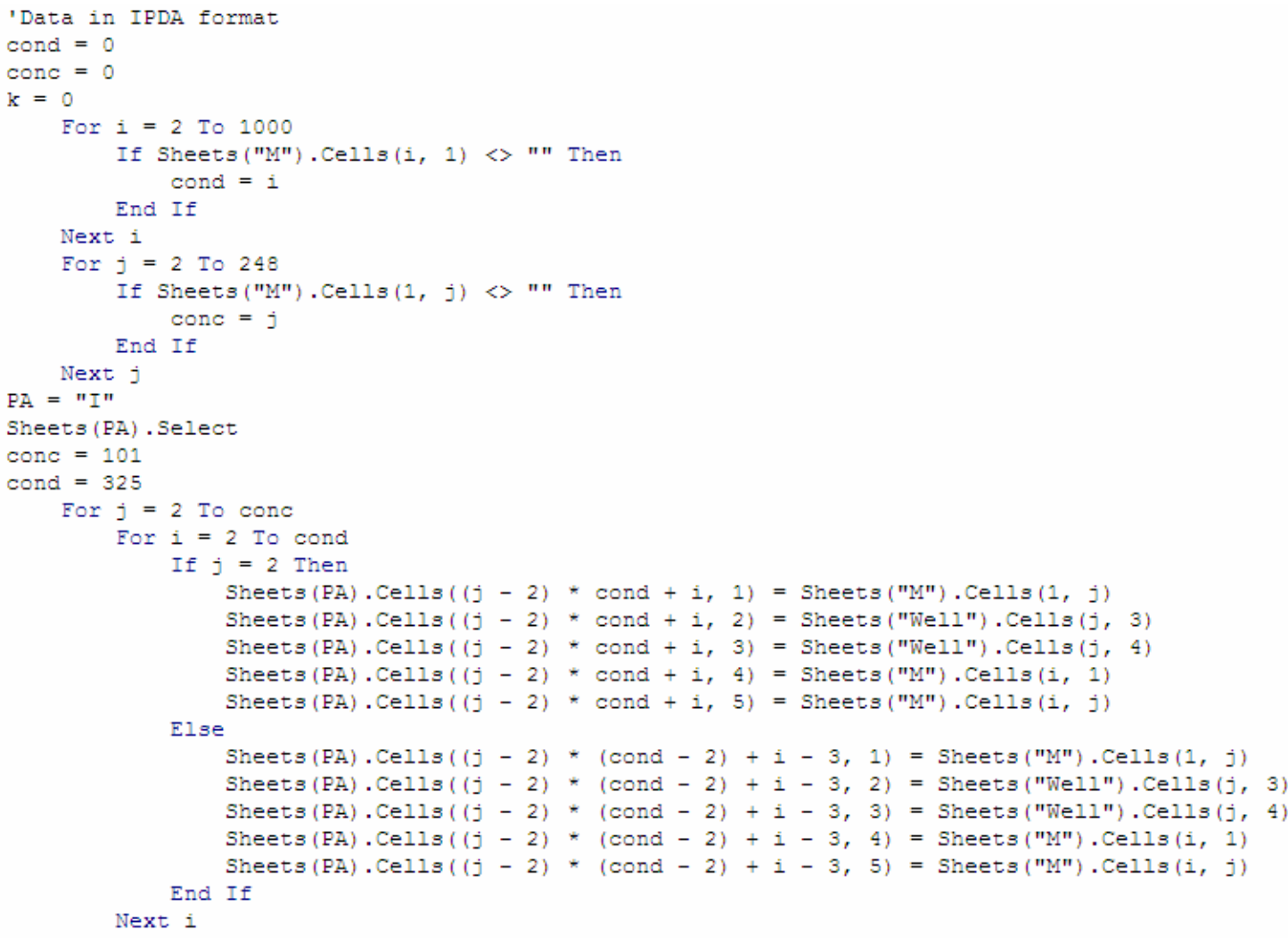

End Sub 